\title{
Numerical Study of Coherent Structures of Turbulence with Large Wall-Normal Gradients in Thermophysical Properties
}

\author{
by \\ Shawn K. Reinink \\ A thesis submitted to the Faculty of Graduate and Postdoctoral Affairs \\ in partial fulfillment of the requirements for the degree of
}

\author{
Master of Applied Science \\ in \\ Aerospace Engineering \\ Carleton University \\ Ottawa, Ontario, Canada
}

(c) 2014

Shawn K. Reinink 


\section{Abstract}

Forced-convection heat transfer in a heated working fluid at a thermodynamic state near its pseudocritical point is poorly predicted by correlations calibrated with data at subcritical conditions. This is primarily due to the influence of large wall-normal thermophysical property gradients that develop in proximity of the pseudocritical point on the concentration of coherent turbulence structures near the wall. The physical mechanisms dominating this influence remain poorly understood. In the present study, direct numerical simulation is used to study the development of turbulence structures within a turbulent spot, which is a more controlled turbulence environment than a fully-turbulent boundary layer, with large wall-normal property gradients. It is found that during improved heat transfer, wall-normal density gradients accelerate the growth of the Kelvin-Helmholtz instability in the shear layer enveloping low-speed streaks through baroclinic vorticity generation. This causes hairpin vortices to form at a faster rate and to mutually interact more frequently. 


\section{Preface}

The content of this thesis is the reproduction of the journal paper listed below, which is currently under review by the journal. This paper was reformatted to meet the thesis requirements at Carleton University. Readers who wish to cite sections of this thesis are strongly encouraged to cite this journal paper following its publication.

Reinink, S. K. and Yaras, M. I. (2014). Numerical study of coherent structures of turbulence with large wall-normal gradients in thermophysical properties. Submitted to Physics of Fluids. 


\section{Acknowledgments}

First, I must thank my supervisor, Dr. Metin I. Yaras, for his instruction, patience, advice, and support. I feel like I learned far more during about two years of research under his supervision than during five years of undergraduate studies (including a co-op term). I would like to thank Chukwudi Azih and Joshua Brinkerhoff, who were senior Ph.D. students in our research group at the time I started my graduate studies. Their guidance played a substantial role in helping me quickly get up to speed as I began my research, and it was also primarily their work which provided the foundation for the present study. I am grateful to my fellow students and colleagues for not only their assistance, but also their friendship; these include Andrew Copping, Derek Ancrum, Hristo Valtchanov, Camelia Nisioiu, Sathya Anand, Dong Jia, Mustafa Siddiqui, Siddharth Kedare, and Kirk Hovell, among others. I am very appreciative of Neil McFadyen and Bruce Johnston for their technical assistance in maintaining and ensuring proper operation of the computational resources of our research group, as well as Irene Helder for her administrative assistance. Finally, I certainly must also include my parents, Kasper and Martha Reinink, and my sister, Kristen, to whom I am very grateful for their continual love, support, and encouragement throughout my undergraduate and graduate studies.

Financial support for the present study was provided by Natural Sciences and Engineering Research Council of Canada (NSERC) and Atomic Energy of Canada Limited (AECL). 


\section{Table of Contents}

Abstract $\quad$ ii

Preface $\quad$ iii

Acknowledgments $\quad$ iv

List of Tables viii

List of Figures $\quad$ ix

Nomenclature $\quad$ xiii

1 Introduction 1

2 Literature Review $\quad 4$

2.1 Influence of large wall-normal gradients in thermophysical properties on forced convection heat transfer . . . . . . . . . . . . . . . 4

2.2 Formation and growth of vortical structures of turbulence in turbulent

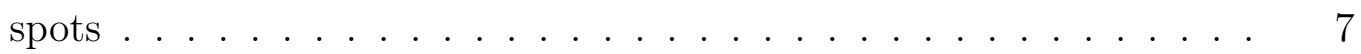

2.3 Instability of free shear layers with cross-stream density gradients . . 10

3 Computational Setup $\quad 15$

3.1 Development of turbulence structures in a turbulent spot with wallnormal property gradients . . . . . . . . . . . . . . . . 15 
3.1 .1 Computational domain . . . . . . . . . . . . . . 15

3.1 .2 Initial and boundary conditions . . . . . . . . . . 17

3.1 .3 Spatial grid . . . . . . . . . . . . . . . . 20

3.2 Free shear layer instability with cross-stream density and viscosity gradients . . . . . . . . . . . . . . . . . . 23

3.3 Solution method $\ldots \ldots \ldots \ldots \ldots \ldots \ldots$

3.4 Validation of the numerical approach . . . . . . . . . 28

4 Results and Discussion $\quad 30$

4.1 Undisturbed laminar flow field in the turbulent spot simulations . . . 30

4.2 Turbulent spot development . . . . . . . . . . . . . . . . . . 34

4.3 Effects of spatial density gradients on the formation of hairpin vortices and their mutual interactions $\ldots \ldots \ldots \ldots$

4.4 Effects of cross-stream density and viscosity gradients on the K-H instability mechanism . . . . . . . . . . . . . . . . . . 51

4.5 Turbulent spot spreading rates . . . . . . . . . . . . . . 60

5 Conclusions and Recommendations $\quad 63$

5.1 Conclusions . . . . . . . . . . . . . . . . . . . . 63

5.2 Recommendations for future work . . . . . . . . . . . . . 64

$\begin{array}{ll}\text { References } & 65\end{array}$

Appendix A Derivation of the Expression for the Rate of Baroclinic $\begin{array}{ll}\text { Vorticity Generation } & 73\end{array}$

$\begin{array}{ll}\text { Appendix B Data Analysis Procedures } & 76\end{array}$

B.1 Perturbation quantities . . . . . . . . . . . 76

B.2 Differential flow parameters . . . . . . . . . . . 77 
B.3 Integral flow parameters . . . . . . . . . . . . . . . . 78

B.4 Identification of coherent vortical structures of turbulence . . . . . . 79 


\section{List of Tables}

3.1 Simulation parameters in Cases 1 and 2 . . . . . . . . . . . . 19

3.2 Node spacings in Cases 1 and $2 \ldots . . \ldots 20$

3.3 Number of nodes in Cases 1 and 2 . . . . . . . . . . . . . . . 22

3.4 Two-dimensional free shear layer simulations . . . . . . . . . . . . . . 24

3.5 Node distributions used in direct numerical simulation studies of a turbulent boundary layer . . . . . . . . . . . . . . . . . . . . . . . . 28

4.1 Strouhal number of $\mathrm{K}-\mathrm{H}$ vortex shedding . . . . . . . . . . . . 55

4.2 Turbulent spot spreading rates with and without a favourable streamwise pressure gradient . . . . . . . . . . . . . . . 62 


\section{List of Figures}

2.1 Variations of selected thermophysical properties of water near the pseudocritical temperature at a supercritical pressure of $\mathrm{P}=24 \mathrm{MPa}$; 'pc' denotes the value at the pseudocritical point . . . . . . . . . 5

2.2 Illustration of the roll-up of (a) a free shear layer and (b) a shear layer enveloping a low-speed streak . . . . . . . . . . . . . . . . . 10

2.3 Illustration of the physical mechanism of baroclinic vorticity generation 11

2.4 Schematic of K-H vortices which have formed in a free shear layer with (a) a uniform density field and (b) a cross-stream density gradient which results in baroclinic vorticity generation. Negative and positive vorticity is shown by solid and dashed lines, respectively. . . . . . . . 12

2.5 Illustration of the formation of Holmboe vortices in a free shear layer exposed to cross-stream density gradients . . . . . . . . . . . . 13

3.1 Computational domain (not to scale) and boundary conditions for Case 116

3.2 Wall-normal node distribution . . . . . . . . . . . . . . . 21

3.3 Computational domain for free shear layer instability simulations . . 23

3.4 Computed (a) turbulence production and dissipation rates, and (b) mean velocity profile. Adapted from Brinkerhoff and Yaras (2011b). . 29

4.1 Streamwise distributions of the freestream velocity, undisturbed boundary layer displacement thickness, and acceleration parameter in

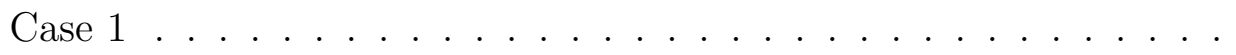


4.2 Undisturbed wall-normal profiles of density, viscosity, thermal conductivity, isobaric specific heat capacity, and temperature in Case 1 at the streamwise location of the jet orifice . . . . . . . . . . . .

4.3 Undisturbed wall-normal profiles of gradients in density, viscosity, thermal conductivity, isobaric specific heat capacity, and temperature in Case 1 at the streamwise location of the jet orifice . . . . . . . .

4.4 Profiles of undisturbed streamwise velocity in Cases 1 and 2 at the streamwise location of the jet orifice . . . . . . . . . . . . 33

4.5 Visualization of the jet-main-flow interaction in Case 1 at the last timestep of the jet pulse using iso-surfaces of $Q=2 \times 10^{-2} \ldots$. . .

4.6 Visualization of hairpin vortices during the early development of the spot in Case 1 using iso-surfaces of $Q=2 \times 10^{-4} \ldots$. . . . . . . . 37

4.7 Illustration of the development of new hairpin vortices from a parent hairpin vortex with (a)-(b) widely-spaced legs and (c)-(d) closelyspaced legs; adapted from Brinkerhoff and Yaras (2014) . . . . . . . . 38

4.8 Illustration of the lateral spreading of the spot by the generation of hairpin vortices along the spot spanwise edges; adapted from Brinkerhoff and Yaras (2014) . . . . . . . . . . . .

4.9 Visualization of the turbulent spots in Cases 1 and 2 using iso-surfaces of $Q=2 \times 10^{-4} \ldots \ldots \ldots \ldots \ldots \ldots \ldots$

4.10 Formation of the B1 hairpin vortex in Case 1. (a)-(c): yz planes at $x / \delta_{0}^{*}=19, \tau=46$ of (a) temperature and streamwise component of perturbation velocity $(\mathrm{u})$, (b) density gradients in the wall-normal and spanwise directions, and (c) components of perturbation vorticity $(\omega)$ in the wall-normal and spanwise directions. (d)-(f): iso-surfaces of $Q=2 \times 10^{-3}$ at $(\mathrm{d}) \tau=46,(\mathrm{e}) \tau=69$, and $(\mathrm{f}) \tau=104 . \ldots$ 
4.11 Formation of the C2 hairpin vortex in Case 1. (a)-(c): yz planes at $x / \delta_{0}^{*}=41, \tau=75$ of (a) temperature and streamwise component of perturbation velocity $(\mathrm{u})$, (b) density gradients in the wall-normal and spanwise directions, and (c) components of perturbation vorticity $(\omega)$ in the wall-normal and spanwise directions. (d)-(f): iso-surfaces of $Q=2 \times 10^{-3}$ at $(\mathrm{d}) \tau=63,(\mathrm{e}) \tau=75$, and $(\mathrm{f}) \tau=109 . \ldots$

4.12 xz plane at $y / \delta_{0}^{*}=1.25$ showing low-speed steak stability in (a) Case 1 at $\tau=347$ and (b) Case 2 at $\tau=442$, when the spots are of similar size 44

$4.13 \mathrm{xz}$ plane at $y / \delta_{0}^{*}=1.25, \tau=347$ showing spanwise density gradients (colour contours) and wall-normal perturbation vorticity (line contours) in Case $1 \ldots \ldots \ldots \ldots \ldots \ldots$

4.14 Hairpin vortices B1 and B2 immediately before they merge into hairpin vortex B1-B2 in the variable-properties case at $\tau=118$ shown with (a) iso-surfaces of $Q=2 \times 10^{-4}$ and (b) the streamwise component of perturbation velocity in the xz plane at $y / \delta_{0}^{*}=1.25 \ldots$. . . . . . . . 46

4.15 Development of hairpin vortices E1 and E2 downstream of B1-B2 in the variable-properties case at $\tau=175$ presented through (a) iso-surfaces of $Q=2 \times 10^{-4}$ and (b) the streamwise component of perturbation velocity in the xz plane at $y / \delta_{0}^{*}=1.5 \ldots \ldots$. . . . . . . . . 47

4.16 Interactions between hairpin vortices in the turbulent spot of Case 1, presented using iso-surfaces of $Q=2 \times 10^{-4}$ and the streamwise component of perturbation velocity in the $\mathrm{xz}$ plane at $y / \delta_{0}^{*}=1.5 \ldots \ldots$

4.17 Streamwise spacing between hairpin vortices in the case with constant properties shown using iso-surfaces of $Q=2 \times 10^{-4}$ and the streamwise component of perturbation velocity in the xz plane at $y / \delta_{0}^{*}=1.5$. .

4.18 Schematic comparing the topology of hairpin vortices in the spot with variable properties (Case 1) and constant properties (Case 2) . . . . 
4.19 Wall-normal profile of streamwise velocity in the high-speed stream at the splitter-plate trailing edge . . . . . . . . . . . . . . . 52

4.20 Illustration of the effect of a wall-normal density gradient on the wallnormal streamwise velocity profile (not to scale) . . . . . . .

4.21 Cross-stream distributions of density and viscosity and their crossstream gradients . . . . . . . . . . . . . . . .

4.22 Development of the free shear layer visualized through the spanwise component of vorticity, $\Omega_{z} \cdot x / L=0$ : splitter-plate trailing edge. . . 54

4.23 Streamwise variation of the power spectral density of streamwise velocity fluctuations at the shedding frequency of the $\mathrm{K}-\mathrm{H}$ vortices evaluated at $y / \theta_{s}=2 \ldots \ldots \ldots \ldots \ldots$

4.24 Illustration of the baroclinic vorticity generation mechanism by which cross-stream density gradients affect the roll-up of a free shear layer into $\mathrm{K}-\mathrm{H}$ vortices . . . . . . . . . . . . . . . .

4.25 Streamwise variation of (a) turbulent spot length and (b) turbulent spot half-width. $x_{T E}$ : streamwise location of the spot trailing edge. . 


\title{
Nomenclature
}

\author{
$\vec{a} \quad$ acceleration vector \\ $C_{p} \quad$ isobaric specific heat capacity \\ D jet orifice hydraulic diameter \\ f $\quad$ vortex shedding frequency \\ G mass flux in the boundary layer \\ $J \quad$ Richardson number, $J=|\vec{a}| \frac{\rho_{1}-\rho_{2}}{\rho_{1}} \frac{U_{1}-U_{2}}{(\partial U / \partial y)_{\max }} \frac{1}{\left(U_{1}-U_{2}\right)^{2}}$ \\ $k \quad$ thermal conductivity \\ $L \quad$ streamwise length of the no-slip splitter plate \\ $L_{x} \quad$ turbulent spot streamwise length \\ $L_{z} \quad$ turbulent spot spanwise half-width \\ $N \quad$ number of grid nodes \\ $P \quad$ static pressure; \\ turbulence kinetic energy production rate \\ $\operatorname{Pr} \quad$ Prandtl number, $\operatorname{Pr}=C_{p} \mu / k$
}


Q second invariant of the velocity-gradient tensor

$q_{w}^{\prime \prime} \quad$ heat flux at the wall

$q_{w}^{\prime \prime} / G \quad$ heat flux-to-mass flux ratio

$R \quad$ free shear layer velocity ratio, $R=\left(U_{1}-U_{2}\right) /\left(U_{1}+U_{2}\right)$

$R_{\text {jet }} \quad$ jet-to-main-flow velocity ratio, $R_{\text {jet }}=V_{\text {jet }} / U_{0}$

$\operatorname{Re}_{\delta} \quad$ Reynolds number based on boundary layer thickness, $\operatorname{Re}_{\delta}=U \delta / \nu$

$\operatorname{Re}_{\delta^{*}} \quad$ Reynolds number based on boundary layer displacement thickness, $\operatorname{Re}_{\delta^{*}}=U \delta^{*} / \nu$

$\operatorname{Re}_{\theta} \quad$ Reynolds number based on boundary layer momentum thickness, $\operatorname{Re}_{\theta}=U \theta / \nu$

St Strouhal number of vortex shedding, St $=f \theta_{s} / U_{K H}$

T temperature

$t \quad$ time

U streamwise component of velocity

U velocity vector

$U_{0} \quad$ undisturbed freestream velocity at the jet orifice

$U_{K H} \quad$ convection speed of $\mathrm{K}-\mathrm{H}$ vortices

$U_{\infty} \quad$ local freestream velocity

$u \quad$ streamwise component of perturbation velocity 
$\mathbf{u}$

$u_{i}, u_{j} \quad$ components of the perturbation velocity vector, where Einstein

summation is applied over the $i$ and $j$ indices

Wall-normal component of velocity

$v \quad$ wall-normal component of perturbation velocity

W $\quad$ spanwise component of velocity

$w \quad$ spanwise component of perturbation velocity

$x \quad$ streamwise spatial coordinate

x spatial coordinate vector

y wall-normal spatial coordinate

$z \quad$ spanwise spatial coordinate

$\delta \quad$ velocity boundary layer thickness

$\delta^{*} \quad$ boundary layer displacement thickness

$\delta_{0}^{*} \quad$ undisturbed boundary layer displacement thickness at the jet orifice

$\epsilon \quad$ turbulence kinetic energy dissipation rate

$\eta \quad$ streamwise acceleration parameter, $\eta=\frac{\nu}{U_{\infty}^{2}} \frac{d U_{\infty}}{d x}$

Kolmogorov length scale

$\theta \quad$ boundary layer momentum thickness

$\theta_{s} \quad$ free shear layer momentum thickness 


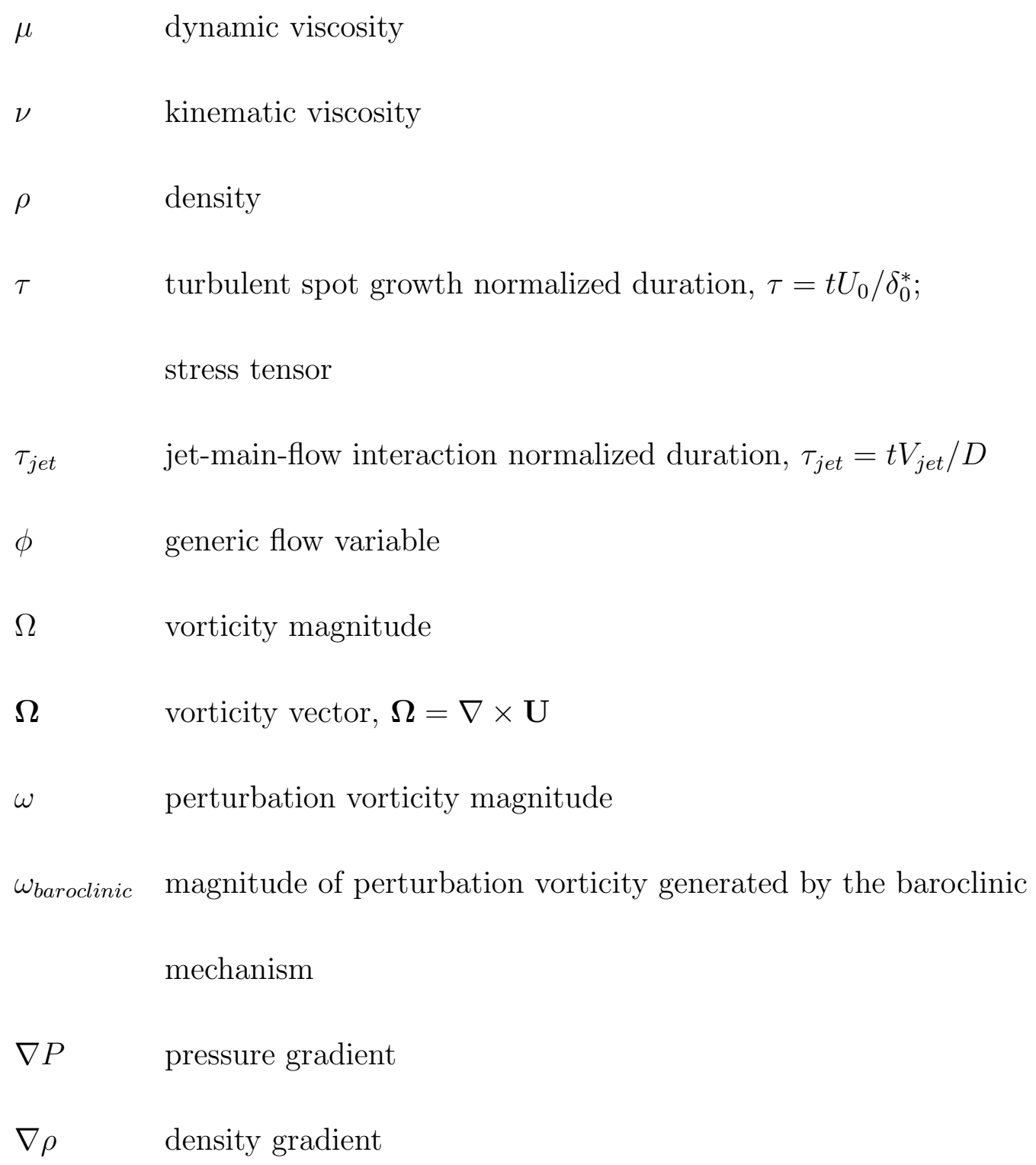

\section{Subscripts}

0 reference value evaluated at the jet orifice location

1 parameter on the high-speed stream side of the free shear layer

2 parameter on the low-speed stream side of the free shear layer

crit critical value 
$i, j \quad$ indices for the $\mathrm{x}, \mathrm{y}$, and $\mathrm{z}$ coordinate directions

in condition at inflow boundary

jet jet flow condition

$p c \quad$ value at the pseudocritical point

$T E$ value at the turbulent spot trailing edge

wall value at the no-slip surface

$x \quad$ component in the streamwise spatial direction

y component in the wall-normal spatial direction

$z \quad$ component in the spanwise spatial direction

$\infty \quad$ value in the undisturbed freestream

\section{Superscripts}

+ parameter normalized by viscous wall parameters 


\section{Chapter 1}

\section{Introduction}

Supercritical fluids have been used in engineering applications throughout the past half-century, and interest in their use continues to grow because of the associated benefits. Advanced thermodynamic cycles for power generation applications are increasingly using supercritical water as the pressures and temperatures in these cycles are pushed higher in the pursuit of improved thermal efficiency. The absence of a phase change in these thermodynamic cycles also allows for a simpler, more compact design which reduces start-up and operating costs and leads to improved safety. For example, in advanced Brayton cycles using supercritical carbon dioxide, the high density of the working fluid entering the compressor brings about a noticeable reduction in power plant size for the required power output. Supercritical working fluids tend to be inexpensive, environmentally friendly, non-explosive, and non-flammable; this makes them excellent candidates for engineering applications including the cooling of electronics and power transmission equipment, and as a refrigerant in air-conditioning and refrigeration systems. It is because of these advantages that one potential next generation nuclear power plant concept, developed as part of the Generation IV nuclear power plant program, aims to use supercritical water as the coolant for nuclear fuel rod bundles (Pioro et al., 2004). 
Engineering systems which use supercritical working fluids must frequently operate in the trans-pseudocritical regime, where small changes in the temperature or pressure of the working fluid near its thermodynamic pseudocritical point lead to significant changes in its thermophysical properties. The forced convection heat transfer rate to a heated working fluid at a thermodynamic state near the pseudocritical point is poorly predicted by empirical correlations calibrated with data at subcritical temperatures and pressures (Pioro et al., 2004). Despite an abundance of experimental data collected over many years, there is still no consensus on the trends and underlying causes of these variations in heat transfer (Pioro et al., 2004). The influence of large thermophysical property gradients in the fluid on convection heat transfer rates is further affected by the flow conditions as defined by the Reynolds number, Grashof number, and heat flux-to-mass flux ratio; the flow geometry in configurations including flat surfaces, curved surfaces, rectilinear channels, and fuel-rod bundles; and, the direction of buoyancy forces in upward, downward, and horizontal flows.

To accurately predict the onset and magnitude of these variations in heat transfer, the underlying flow physics must be fully understood. Direct numerical simulation is a technique which has proven to be very effective in shedding light on the physical mechanisms driving the development of turbulent flows because it involves the resolution of the full range of the spatial and temporal scales of turbulence. The identification of cause-and-effect relationships in such fundamental studies is best realized by systematically examining the individual effects of fluid, flow, and geometric parameters.

Recent studies have shown that the variations in heat transfer observed when the working fluid is at a thermodynamic state near the pseudocritical point are closely related to variations in the concentration of turbulence structures within the near-wall region of the boundary layer (Bae et al., 2008; Azih et al., 2012; Azih and Yaras, 2013). Accordingly, in the present study direct numerical simulations are used to observe the 
development of coherent vortical structures within a turbulent spot under the influence of large wall-normal property gradients in the absence of buoyancy forces. A turbulent spot rather than a fully-turbulent boundary layer is used for the study, for the coherent structures of turbulence in a spot tend to be in a more organized state which may allow for more effective identification of cause-and-effect relationships (Schröder and Kompenhans, 2004). Simulation of a non-buoyant flow allows for identification of the mechanisms which are independent of the effects of gravitational forces. Large wall-normal gradients in thermophysical properties are created by heating water at a thermodynamic state near the pseudocritical point. Flow developments driven by the growth of the Kelvin-Helmholtz instability in the shear layer at the interface between low-speed streaks and the surrounding higher-speed flow play a substantial role in the generation of turbulence structures in turbulent flows. Therefore, several two-dimensional direct numerical simulations of the roll-up of an isolated free shear layer with large cross-stream thermophysical property gradients are performed to identify their effects on the growth of the Kelvin-Helmholtz instability.

Improved understanding of the physical mechanisms behind variations in convection heat transfer in supercritical fluids acquired through fundamental studies such as the present study provide the foundation for realizing enhancements to engineering and design tools. For example, current turbulence models used in Reynolds-averaged Navier-Stokes simulations are not able to capture the variations in heat transfer rates observed under certain conditions in heated supercritical fluids. Fundamental studies such as the present study will facilitate revisions of current turbulence models to enable their reliable use for such conditions. 


\section{Chapter 2}

\section{Literature Review}

\subsection{Influence of large wall-normal gradients in thermophys- ical properties on forced convection heat transfer}

It has been well-documented over the last sixty years that forced-convection heat transfer in a working fluid at a thermodynamic state near its pseudocritical point with prevailing spatial gradients in its thermophysical properties is poorly predicted by heat transfer correlations calibrated for conditions at subcritical temperatures and pressures (Pioro et al., 2004). Forced-convection heat transfer rates which are under-predicted or over-predicted by these correlations are referred to as improved heat transfer (IHT) and deteriorated heat transfer (DHT), respectively (Pioro et al., 2004). The large changes in the thermophysical properties of water which occur with small changes in temperature near the pseudocritical point are shown in Fig. 2.1. The pseudocritical point is defined as the temperature at which the isobaric specific heat capacity, $C_{p}$, reaches its maximum value at the fluid pressure.

Azih and Yaras (2013) used a direct numerical simulation of forced-convection heat transfer in a heated channel with supercritical water in the absence of buoyancy effects to observe that the reduction in the Nusselt number during DHT due to changes in turbulent mixing is an order of magnitude greater than the reduction 


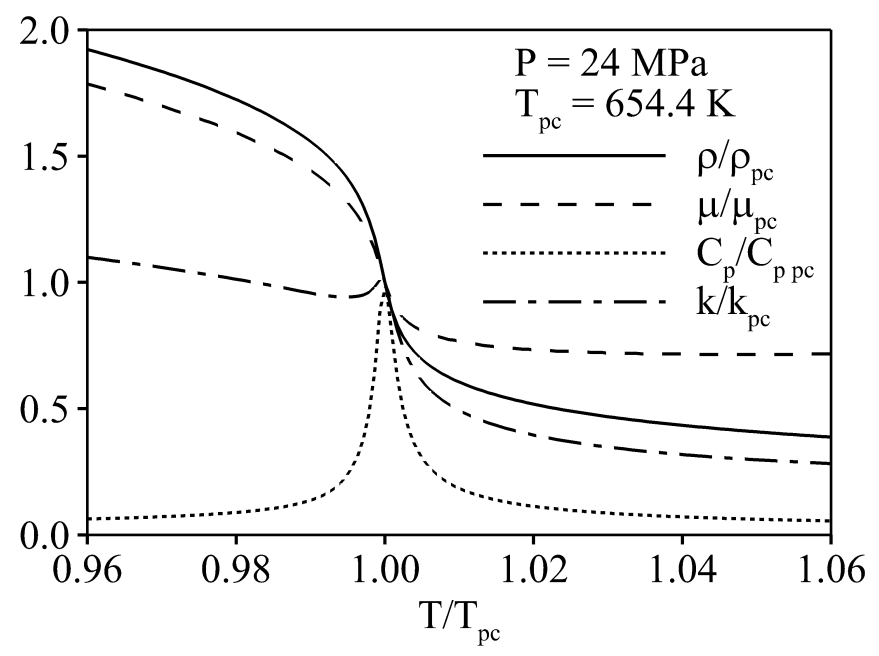

Figure 2.1: Variations of selected thermophysical properties of water near the pseudocritical temperature at a supercritical pressure of $\mathrm{P}=24 \mathrm{MPa}$; 'pc' denotes the value at the pseudocritical point

due to molecular thermal diffusivity. Bae et al. (2008) and Azih et al. (2012) showed that DHT and IHT are primarily caused by variations in the concentration of coherent turbulence structures in the near-wall region of the boundary layer. Azih et al. (2012) and Azih and Yaras (2013) hypothesized that these variations are driven by the influence of spatial gradients in density and viscosity on the local stability of the boundary layer. Azih and Yaras (2013) suggested that for supercritical fluids flowing over heated walls, the wall-normal viscosity gradient stabilizes the flow by working against inviscid instabilities necessary for the regeneration of coherent turbulence structures, while the wall-normal density gradient promotes inviscid instability. Furthermore, in channels which are heated to produce sufficiently large streamwise gradients in wall temperature, the decrease in the near-wall fluid density with streamwise distance results in streamwise acceleration near the wall which stabilizes the shear layer. During DHT, Azih and Yaras (2013) proposed that the destabilizing effects of the wall-normal density gradient are more than offset by the 
stabilizing effects of the wall-normal viscosity gradient and streamwise density gradient. However, the details of the physical mechanisms by which spatial gradients in density and viscosity affect the development and concentration of turbulence structures remain poorly understood, largely because of the relatively disorganized state of the coherent turbulence structures in a fully-turbulent boundary layer (Azih et al., 2012). Conversely, studies which focus on the influence of spatial gradients in thermophysical properties on boundary layer stability are limited to using linear stability analysis and experimentally determining the point of transition in flows that are heated or cooled near standard atmospheric pressure and temperature (Wazzan et al., 1972; Özgen, 2004). In these studies, the property gradients in the boundary layer are several orders of magnitude lower than in flows which are heated at a thermodynamic state near the pseudocritical point. The results of these studies have shown that the effects of spatial variations in density and viscosity on boundary layer stability are several orders of magnitude greater than those of other thermophysical properties (Schäfer et al., 1995).

A turbulent spot provides a more orderly environment to observe the development of coherent turbulence structures compared to a fully-turbulent boundary layer (Schröder and Kompenhans, 2004). Accordingly, direct numerical simulations are performed in the present study in which the internal structure of a turbulent spot on a heated flat plate in a working fluid at a thermodynamic state near the pseudocritical point is investigated to shed light on the physical mechanisms by which large spatial gradients in density and viscosity affect the development and mutual interaction of coherent structures of turbulence and the resultant rates of forced-convection heat transfer. The results of the present study show the effects of spatial gradients in density to be dominant compared to those of viscosity at the test conditions which are simulated. Spatial gradients in density are therefore the focus of this thesis. 


\subsection{Formation and growth of vortical structures of turbu- lence in turbulent spots}

A turbulent spot is a localized region of turbulent flow within a laminar shear layer which is created by the growth of a local disturbance. The existence of turbulent spots was first observed by Emmons (1951) in a shallow water channel. A turbulent spot can develop starting from the presence of a single hairpin vortex (Singer and Joslin, 1994), or it can appear in a semi-developed state if initiated by a sufficiently strong disturbance (Katz et al., 1990). Turbulent spots have been studied extensively using direct numerical simulation (Henningson et al., 1987; Singer, 1996), hot wire measurements (Schubauer and Klebanoff, 1956; Sankaran et al., 1986), flow visualization methods (Perry et al., 1981; Matsui, 1980), and particle image velocimetry techniques (Schröder and Kompenhans, 2004; Schröder et al., 2008). In studies focusing on the structure of turbulent spots, spots are typically triggered artificially using a jet or pulse of working fluid ejected from the wall (Gad-el-Hak et al., 1981; Yaras, 2007); other methods include the use of a spark discharge (Wygnanski et al., 1976) or a roughness element (Chong and Zhong, 2003). The identifying characteristics of a turbulent spot are independent of the disturbance that creates it once the spot has travelled a sufficient distance downstream from the disturbance (Perry et al., 1981; Wygnanski et al., 1976). A turbulent spot typically has the shape of a curved arrowhead pointing downstream (Schubauer and Klebanoff, 1956; Wygnanski et al., 1976). The leading edge of the spot (the point of the arrowhead) consistently rises from the wall, creating a turbulent region which overhangs a region of laminar flow (Schubauer and Klebanoff, 1956). Zhong et al. (1998), Wu et al. (1999), and Yaras (2007) observed spots shaped as arrowheads pointing upstream. Wu et al. (1999) suggested this occurs if the disturbance is dominant in the upper part of the boundary layer. The growth rate and shape of the spot are dependent on the flow Reynolds number, 
streamwise pressure gradient, surface roughness, and level of freestream turbulence (Gostelow et al., 1996). A region of increased stability is present immediately upstream of the trailing edge of the spot (Schubauer and Klebanoff, 1956; Wygnanski et al., 1976).

Flow visualization studies have shown that turbulent spots are densely populated with arrays of hairpin-shaped vortices similar to those observed within fully-turbulent boundary layers (Perry et al., 1981; Matsui, 1980; Schröder et al., 2008). The physical mechanisms by which turbulence structures regenerate within a turbulent spot are also the same as in a fully-turbulent boundary layer (Singer and Joslin, 1994; Singer, 1996; Schröder and Kompenhans, 2004). Locally-induced upwash and downwash motions associated with first-generation or primary hairpin vortices lead to the development of streamwise-oriented streaks of low-speed and high-speed flow near the wall (Wygnanski, 1981; Schröder and Kompenhans, 2004; Yaras, 2007). In the shear layer that forms at the interface between the low-speed streaks and the surrounding higher-speed flow, inflectional streamwise velocity profiles develop in the streamwise-spanwise plane and in the streamwise-wall-normal plane (Swearingen and Blackwelder, 1987). Secondary hairpin vortices form upstream of existing primary hairpin vortices due to the inviscid-unstable state of this shear layer which rolls it up into hairpin vortices through the Kelvin-Helmholtz $(\mathrm{K}-\mathrm{H})$ instability mechanism. Smith et al. (1991) and Singer and Joslin (1994) observed the formation of secondary hairpin vortices where the shear layer enveloping low-speed streaks rolls up into spanwise-oriented vortices above the streaks immediately upstream of their respective primary hairpin vortices. The ends of the spanwise vortices proceed to bend downward around the low-speed streak, forming into secondary hairpin vortices between the legs of the primary hairpin vortices. Schröder and Kompenhans (2004) and Schröder et al. (2008) observed the formation of secondary hairpin vortices in which the shear layer along low-speed streaks rolls up into wall-normal vortices on the 
sides of the streaks. These wall-normal vortices fold over the legs of their respective primary hairpin vortices, resulting in a topology in which secondary hairpin vortices straddle the legs of primary hairpin vortices.

The generation of new hairpin vortices upstream of existing hairpin vortices in this manner leads to groups of hairpin vortices called wave packets, in which hairpin vortices are arranged in streamwise succession and travel downstream together at about the same speed (Zhou et al., 1999; Adrian et al., 2000; Ganapathisubramini et al., 2003). Wave packets in turbulent boundary layers and turbulent spots at displacement-thickness-based Reynolds number values of $\operatorname{Re}_{\delta^{*}} \sim O\left(10^{2}\right)$ are generally arranged in groups of two to three hairpin vortices; the number of hairpin vortices in a wave packet increases to about four to five for $\operatorname{Re}_{\delta^{*}} \sim O\left(10^{3}\right.$ ) (Adrian et al., 2000). Hairpin vortices in wave packets in a zero-pressure-gradient boundary layer are characteristically streamwise-spaced several hundred viscous wall units apart (Adrian et al., 2000), and the spanwise spacing between adjacent wave packets is about 100 viscous wall units (Adrian et al., 2000; Smith and Metzler, 1983; Kline et al., 1967). Viscous wall units correspond to the distance normalized by the friction velocity and the kinematic viscosity at the nearest wall. As wave packets convect downstream, the hairpin vortices within them extend in the wall-normal direction due to self-induced motions and stretch in the streamwise direction due to the background wall-normal profile of streamwise velocity. Older wave packets which are larger in size are exposed to flow with higher streamwise velocity farther from the wall, and therefore travel downstream faster than newer, smaller wave packets. This leads older, larger wave packets to overtake and mutually interact with newer, smaller wave packets (Adrian et al., 2000). 
(a)

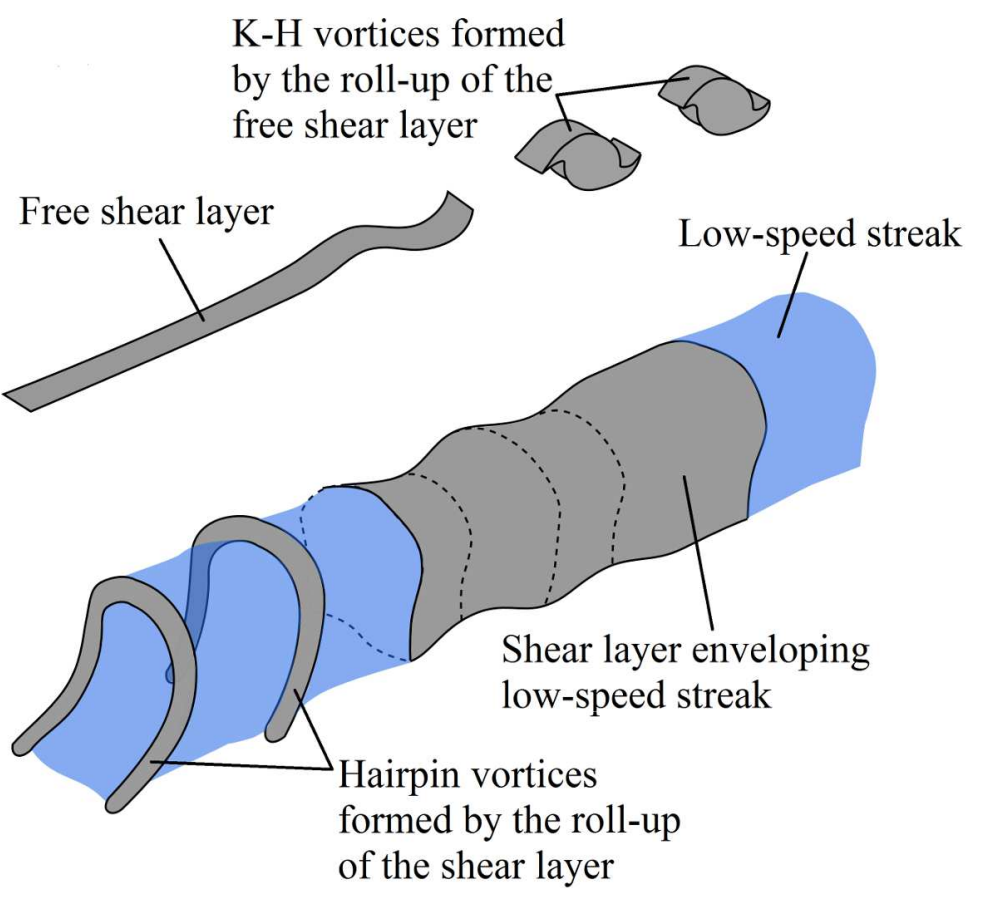

Figure 2.2: Illustration of the roll-up of (a) a free shear layer and (b) a shear layer enveloping a low-speed streak

\subsection{Instability of free shear layers with cross-stream density gradients}

The physical mechanisms by which the K-H instability grows within the shear layer enveloping low-speed streaks leading to the roll-up of the shear layer into hairpin vortices are fundamentally the same as those which occur in the roll-up of a free shear layer into K-H vortices (Zaki and Durbin, 2005; Durbin and Wu, 2007; Brinkerhoff and Yaras, 2014). This is illustrated schematically in Fig. 2.2. Studies have shown that $\mathrm{K}-\mathrm{H}$ instability growth in a free shear layer is significantly affected by large density gradients (Davey and Roshko, 1972; Turner, 1973; John, 2012). In the present study, it is hypothesized that spatial gradients in density may affect the shear layer enveloping low-speed streaks and the formation of hairpin vortices by similar physical mechanisms. For flows with a Froude number value much greater than one, the 


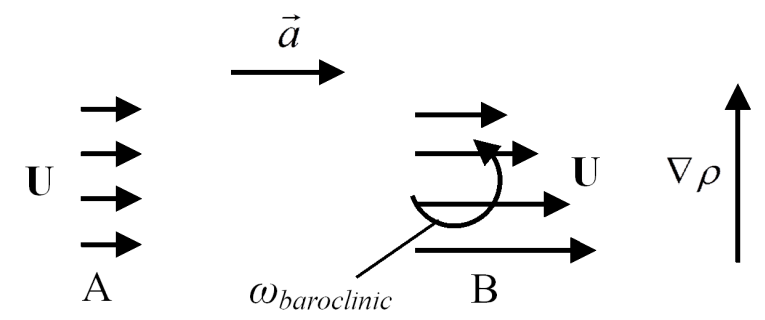

Figure 2.3: Illustration of the physical mechanism of baroclinic vorticity generation developing instabilities in the free shear layer are dominated by inertial effects and the influence of gravity is insignificant (Davey and Roshko, 1972). In such flows, the two primary mechanisms by which cross-stream density gradients influence the roll-up of a free shear layer into K-H vortices are baroclinic vorticity generation and the Holmboe instability mode (Turner, 1973; Soteriou and Ghoniem, 1995; Reinaud et al., 2000; John, 2012).

The rate of baroclinic vorticity generation is described by the following expression:

$$
\frac{D \omega_{\text {baroclinic }}}{D t}=\frac{1}{\rho}\left(\frac{D \mathbf{U}}{D t} \times \nabla \rho\right)
$$

where $D / D t$ is the material derivative, $\mathbf{U}$ denotes the velocity vector, and $\nabla \rho$ represents the gradient of density. The derivation of this expression is provided in Appendix A. The physical mechanism of baroclinic vorticity generation is shown in Fig. 2.3. Fluid particles in the figure are accelerated from point $\mathrm{A}$ to $\mathrm{B}$ in the direction of fluid motion as shown by the $\vec{a}$ vector. A positive cross-stream density gradient exists as shown by the $\nabla \rho$ vector such that fluid particles at the bottom of the figure have a lower density than those at the top of the figure. The fluid particles in the flow field at a lower density are accelerated to a higher velocity than those at a higher density, thus generating vorticity. The effect of baroclinic vorticity generation on the vorticity distribution within $\mathrm{K}-\mathrm{H}$ vortices through the $\mathrm{K}-\mathrm{H}$ instability mode 


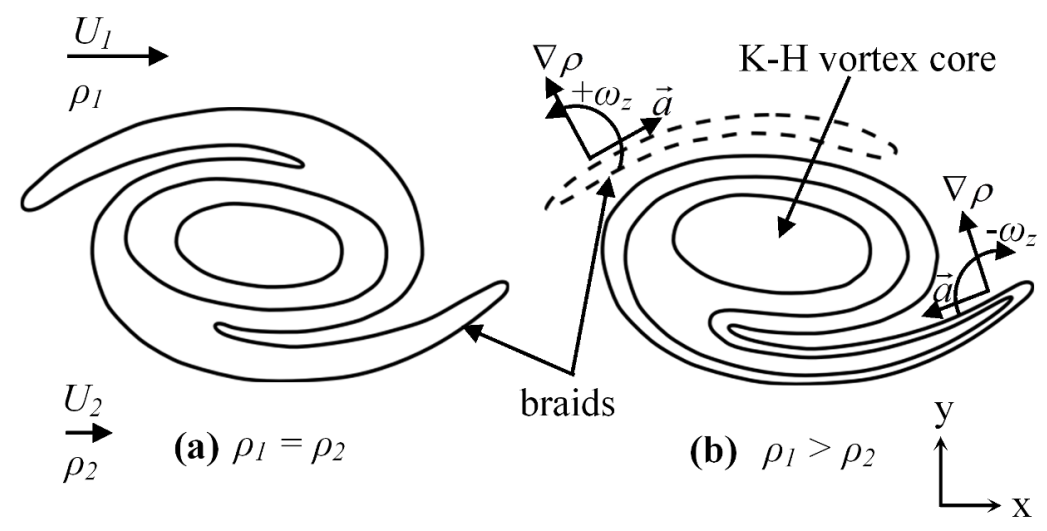

Figure 2.4: Schematic of K-H vortices which have formed in a free shear layer with (a) a uniform density field and (b) a cross-stream density gradient which results in baroclinic vorticity generation. Negative and positive vorticity is shown by solid and dashed lines, respectively.

which have formed by the roll-up of a free shear layer is shown in Fig. 2.4. Vector $\vec{a}$ is the rate of acceleration in the direction of local fluid motion; $\omega_{z}$ represents the spanwise vorticity generated by the baroclinic mechanism; $\rho_{1}$ and $\rho_{2}$ denote the density of the high-speed and low-speed streams; and, $U_{1}$ and $U_{2}$ denote the velocity of the high-speed and low-speed streams, respectively. Fluid particles in the braids, which are the thin layers of spanwise vorticity labelled in Fig. 2.4, are accelerated along the braids toward the K-H vortex core (Reinaud et al., 1999, 2000). Soteriou and Ghoniem (1995), Staquet (1995), and Reinaud et al. (1999, 2000) each observed that this acceleration results in the production of positive vorticity along the braid on the higher-density side of the shear layer by baroclinic vorticity generation which partially cancels the existing background negative vorticity that is present. Similarly, negative vorticity is produced along the braid on the lower-density side of the shear layer which adds to the background negative vorticity within the shear layer.

The second mechanism observed in literature by which cross-stream density gradients affect the roll-up of a free shear layer into K-H vortices is the Holmboe instability mode. John (2012) performed a direct numerical simulation of a free shear layer with 


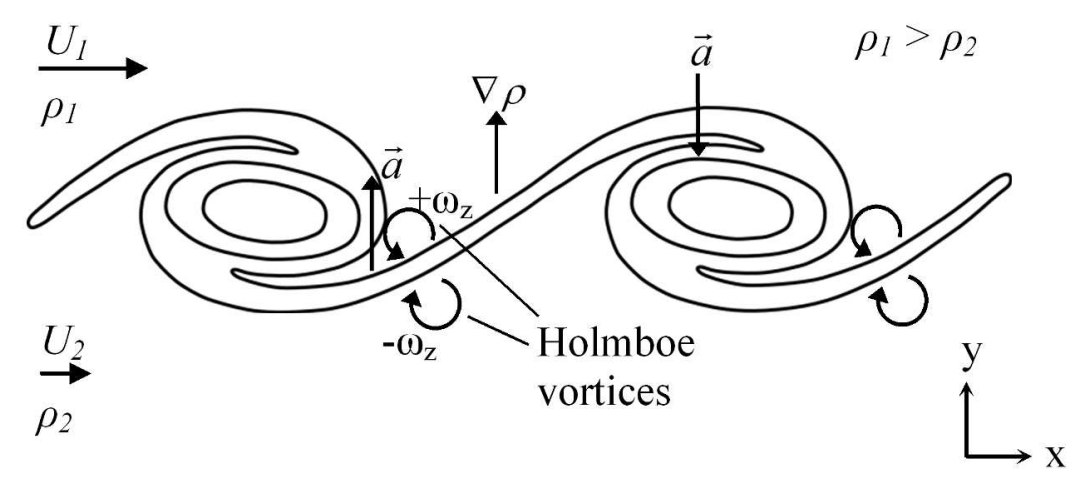

Figure 2.5: Illustration of the formation of Holmboe vortices in a free shear layer exposed to cross-stream density gradients

large cross-stream property gradients created between two streams of water of different velocity and temperature values at a thermodynamic state near the pseudocritical point. The results of the simulation show that as the free shear layer rolls up into $\mathrm{K}-\mathrm{H}$ vortices by the $\mathrm{K}-\mathrm{H}$ instability mechanism, it develops a streamwise-wavy pattern at the dominant wavelength of instability. The resulting curvature in the shear layer causes local fluid particles to experience centripetal acceleration normal to the local direction of fluid motion. In locations where the centripetal acceleration is in the same direction as the positive density gradient, this results in the formation of a pair of counter-rotating vortical structures of spanwise vorticity consistent with the development of Holmboe instability waves as shown in Fig. 2.5. In the figure, $\vec{a}$ is the local centripetal acceleration vector and $\omega_{z}$ is the vorticity contained in the Holmboe vortices. The Richardson number based on the magnitude of the centripetal acceleration, $|\vec{a}|$, is defined as:

$$
J=|\vec{a}| \frac{\rho_{1}-\rho_{2}}{\rho_{1}} \frac{U_{1}-U_{2}}{(\partial U / \partial y)_{\max }} \frac{1}{\left(U_{1}-U_{2}\right)^{2}}
$$

For the Holmboe instability to develop, the Richardson number must be between about 0.025 and 0.5 ; the wavenumber based on the streamwise spacing between the 
developing Holmboe vortices must be greater than 0.25 ; and, the ratio of the free shear layer thickness to the thickness over which the density varies must be greater than about 2 to 2.4 (Lawrence et al., 1991; Hazel, 1972; Smyth and Peltier, 1989; John, 2012). In the study of John (2012) the local Richardson number based on the centripetal acceleration experienced by fluid particles approached values as high as $\mathrm{Ri}=0.16$, the ratio of the free shear layer thickness to the thickness over which the density varies had a value of five, and the wavenumber based on the streamwise spacing between Holmboe vortices was 1.2. 


\section{Chapter 3}

\section{Computational Setup}

\subsection{Development of turbulence structures in a turbulent spot with wall-normal property gradients}

The results of the present study are compared to those of a similar study by Brinkerhoff and Yaras (2014), which examines the growth of a turbulent spot with spatially-uniform thermophysical properties. Hereafter, the present turbulent spot simulation will be referred to as Case 1, and the turbulent spot simulation of Brinkerhoff and Yaras (2014) will be referred to as Case 2.

\subsubsection{Computational domain}

Two direct numerical simulations are performed in Case 1, each consisting of water flow over a heated no-slip surface in the absence of buoyancy forces. As was done by Brinkerhoff and Yaras (2014) for Case 2, two smaller simulations are performed to avoid an unnecessarily large computational domain as the turbulent spot develops while propagating in the downstream direction. The computational domains for the two simulations are shown in Fig. 3.1. The turbulent spot is generated in Simulation 1 using a jet of water pulsed normal to the main flow through a square orifice flush with the no-slip surface. The instantaneous flow field at a plane upstream of the trailing 


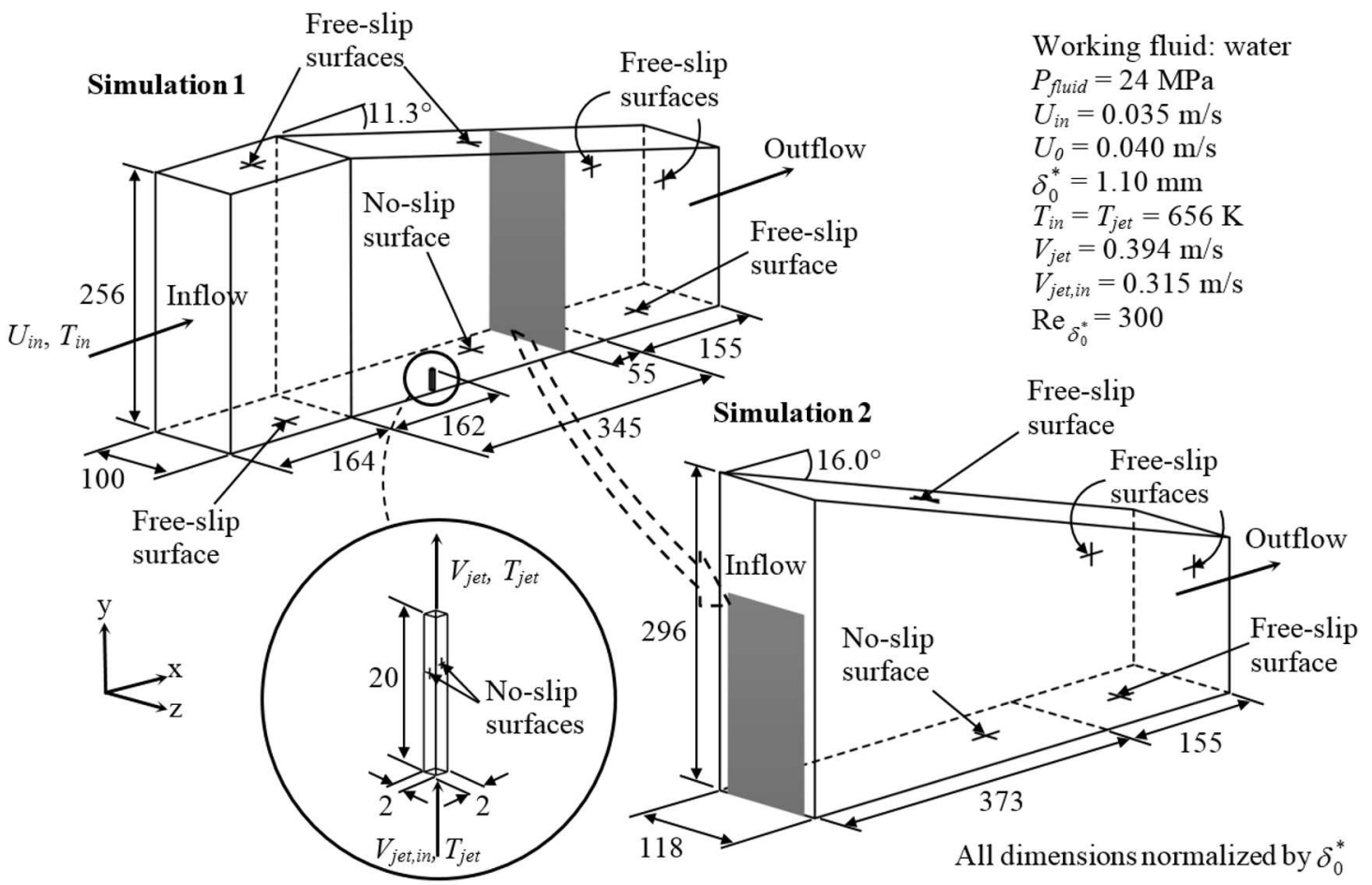

Figure 3.1: Computational domain (not to scale) and boundary conditions for Case 1

edge of the no-slip surface is mapped to the inflow boundary of Simulation 2. $U_{0}$ and $\delta_{0}^{*}$ values listed in Fig. 3.1 correspond to the undisturbed freestream velocity and displacement thickness of the boundary layer at the streamwise location of the jet orifice. $\operatorname{Re}_{\delta_{0}^{*}}$ denotes the flow Reynolds number based on $\delta_{0}^{*}$ and the spatial average of kinematic viscosity over the thickness of the local velocity boundary layer.

The data-mapping plane between the domains of the two simulations is conservatively placed $55 \delta_{0}^{*}$ upstream of the trailing edge of the no-slip surface in Simulation 1. Brinkerhoff and Yaras (2014) demonstrated that placing such a plane at least $28 \delta_{0}^{*}$ upstream of the trailing edge of the no-slip surface is sufficient to prevent the flow at this plane from being artificially influenced by the outflow boundary condition of the computational domain placed $155 \delta_{0}^{*}$ downstream of the trailing edge.

The domain widths in Simulations 1 and 2 are sized so that the closest edge of the spot is more than $29 \delta_{0}^{*}$ from the nearest side boundary such that the spanwise 
growth of the spot is not artificially influenced. The ceiling is placed conservatively far from the no-slip surface so that it does not influence the development of turbulence structures within the turbulent spot, which grow to a maximum height of not more than $15 \delta_{0}^{*}$. Based on the results of preliminary simulations, a favourable streamwise pressure gradient is imposed using a sloped ceiling, beginning at the leading edge of the no-slip surface in Simulation 1. This moderates the pace of development of the turbulent spot so that the coherent turbulence structures in the spot can be more readily observed (Wygnanski, 1981). The slope of the ceiling is increased in Simulation 2 to maintain the same streamwise rate of freestream acceleration over the no-slip surface with a ceiling placed at a greater height.

In Case 2 computed by Brinkerhoff and Yaras (2014), the absence of wall-normal thermophysical property gradients results in a more stable flow environment. Accordingly, they did not require the use of an accelerating freestream. Instead, they established a non-accelerating freestream by matching the streamwise variation of the ceiling height to the streamwise displacement thickness distribution of the Blasius boundary layer.

\subsubsection{Initial and boundary conditions}

Before generating the turbulent spot, a laminar boundary layer is developed on the no-slip surface of Simulations 1 and 2; the simulations are initialized using uniform velocity and temperature values of $U=U_{0}$ and $T=T_{i n}$, respectively. The flow field in the jet channel in Simulation 1 is initialized using velocity and temperature values of $V=0.0 \mathrm{~m} / \mathrm{s}$, and $T=T_{\text {jet }}$.

The development of the laminar boundary layer is computed using uniform inlet velocity and temperature values of $U=U_{0}$ and $T=T_{\text {in }}$ at the inflow boundary of Simulation 1. The data on the mapping plane in Simulation 1 is then used as the 
inflow condition of Simulation 2. The inflow temperature is just above the pseudocritical water temperature of $T_{p c}=654.4 \mathrm{~K}$ at $P=24 \mathrm{MPa}$. As the fluid is heated over the no-slip surface, its state moves further away from the pseudocritical point. This setup ensures that the wall-normal density gradients are not too high to cause the turbulence to develop too quickly which would prevent detailed observation of the flow physics. The outflow boundary in Simulations 1 and 2 is set to a fixed area-averaged pressure value of $24 \mathrm{MPa}$. This outflow boundary condition creates a pressure difference of about $0.35 \mathrm{~Pa}$ between the mapping plane in Simulation 1 and the inflow boundary of Simulation 2. This discrepancy was determined to have a negligible effect on the thermophysical property values and hence the simulation results.

Free-slip surfaces of the computational domains and the jet channel no-slip surfaces are set to zero heat flux. On the no-slip surface over which the main-flow boundary layer develops, the heat flux is increased linearly from $q_{w}^{\prime \prime}=0.0 \mathrm{~kW} / \mathrm{m}^{2}$ at the leading edge to $q_{w}^{\prime \prime}=3.75 \mathrm{~kW} / \mathrm{m}^{2}$ at the streamwise location of the jet orifice, and is held constant at this value along the remainder of the surface. The corresponding heat flux-to-mass flux ratio reduces from $q_{w}^{\prime \prime} / G=0.19 \mathrm{~kJ} / \mathrm{kg}$ at the location of the jet orifice to $q_{w}^{\prime \prime} / G=0.10 \mathrm{~kJ} / \mathrm{kg}$ at the end of the no-slip surface of Simulation 2 . Here, $G$ is the local mass flux in the laminar boundary layer. This range of $q_{w}^{\prime \prime} / G$ and fluid thermodynamic state correspond to conditions at which improved heat transfer typically occurs (Pioro et al., 2004; Azih et al., 2012).

After the steady-flow, steady-state laminar flow field is established, the turbulent spot is generated in Simulation 1 by pulsing the jet for a normalized duration of $\tau_{\text {jet }}=t V_{\text {jet }} / D=173$, where $D$ is the orifice hydraulic diameter. The jet is pulsed using a step change in the uniform inflow velocity field from $V=0.0 \mathrm{~m} / \mathrm{s}$ to $V=V_{\text {jet,in }}$ at the jet channel inflow boundary. No-slip surfaces on the sides of the jet channel allow development of the boundary layer along the jet channel walls. This accelerates 
Table 3.1: Simulation parameters in Cases 1 and 2

\begin{tabular}{lcc}
\hline & Case 1 & Case 2 \\
\hline Working fluid & Water & Air \\
$P_{\text {fluid }}$ & $24 \mathrm{MPa}$ & $101 \mathrm{kPa}$ \\
$U_{\text {in }}$ & $0.035 \mathrm{~m} / \mathrm{s}$ & $4.55 \mathrm{~m} / \mathrm{s}$ \\
$U_{0}$ & $0.040 \mathrm{~m} / \mathrm{s}$ & $4.55 \mathrm{~m} / \mathrm{s}$ \\
$\delta_{0}^{*}$ & $1.10 \mathrm{~mm}$ & $1.44 \mathrm{~mm}$ \\
$T_{\text {in }}=T_{\text {jet }}$ & $656 \mathrm{~K}$ & $298 \mathrm{~K}$ \\
$V_{\text {jet }}$ & $0.394 \mathrm{~m} / \mathrm{s}$ & $44 \mathrm{~m} / \mathrm{s}$ \\
$V_{\text {jet }, \text { in }}$ & $0.315 \mathrm{~m} / \mathrm{s}$ & $35 \mathrm{~m} / \mathrm{s}$ \\
$\tau_{\text {jet }}$ & 173 & 173 \\
$R_{\text {jet }}$ & 9.8 & 9.7 \\
$\operatorname{Re}_{\delta_{0}^{*}}$ & 300 & 430 \\
\hline
\end{tabular}

the jet flow to $V_{\text {jet }}$ at the jet orifice such that the jet-to-main-flow velocity ratio is $R_{\text {jet }}=V_{\text {jet }} / U_{0}=9.8$. This jet velocity ratio activated for $\tau_{\text {jet }}=173$ duration creates a sufficiently strong disturbance to generate a turbulent spot in a laminar boundary layer exposed to a strong favourable streamwise pressure gradient. Simulation 1 is then continued for $\tau=410$ time units, where $\tau=t U_{0} / \delta_{0}^{*}$ and $\tau=0$ corresponds to the first timestep after the jet is turned off. Mapping of the transient flow field from the mapping plane in Simulation 1 to the inflow boundary of Simulation 2 is commenced at $\tau=61$ and is continued until $\tau=410$. The leading edge of the turbulent spot reaches the trailing edge of the no-slip surface in Simulation 2 before the flow field at the inflow boundary fully recovers from the wake of the spot.

Table 3.1 summarizes the parameters used in Case 1 and those used by Brinkerhoff and Yaras (2014) in Case 2. The turbulent spots in the two cases are generated nearly identically at comparable Reynolds numbers. In contrast to the accelerating freestream in Case 1, the ceiling height in Case 2 is set to maintain a non-accelerating 
Table 3.2: Node spacings in Cases 1 and 2

\begin{tabular}{|c|c|c|c|c|}
\hline Simulation & $\Delta x^{+}$ & $\Delta z^{+}$ & $\Delta y_{\text {wall }}^{+}$ & $\operatorname{Re}_{\theta}{ }^{1}$ \\
\hline Case 1, Simulation 1 & 22 & 10 & 0.33 & 160 \\
\hline Case 1, Simulation 2 & 20 & 8 & 0.33 & 160 \\
\hline Case 2, Simulation 1 (Brinkerhoff and Yaras, 2014) & 20 & 7 & 0.70 & 332 \\
\hline Case 2, Simulation 2 (Brinkerhoff and Yaras, 2014) & 20 & 8 & 0.70 & 332 \\
\hline
\end{tabular}

freestream. The flow initialization and boundary conditions are the same for the two cases except for the no-slip surface heat flux, which is set to zero to yield spatially uniform thermophysical properties in Case 2.

\subsubsection{Spatial grid}

The smallest scale turbulence structures in a fully-turbulent boundary layer are on the order of the Kolmogorov length scale, which has a value of $\eta^{+} \approx 2$ close to a no-slip surface (Stanislas et al., 2008). Spatial grid node spacing in Cases 1 and 2 is based on the Kolmogorov length scale to accurately capture the development of turbulence structures within the spot, as presented in Table 3.2.

The viscous length scale used to normalize node spacing values is computed based on a spatial average over the footprint of the turbulent spot at its most mature state in Simulation 2 of each case. The streamwise node spacing in Simulation 1 in the regions above the free-slip surfaces upstream and downstream of the no-slip surface is increased at a rate of less than $15 \%$ until the inflow and outflow boundary is reached, respectively. The streamwise node spacing above the free-slip surface in Simulation 2 is distributed the same way. Node spacing in the immediate vicinity of the jet orifice is decreased at a rate of less than $15 \%$ in the streamwise and spanwise directions to average values of $\Delta x^{+}=7$ and $\Delta z^{+}=4$ in Case 1 , and $\Delta x^{+}=8$ and 


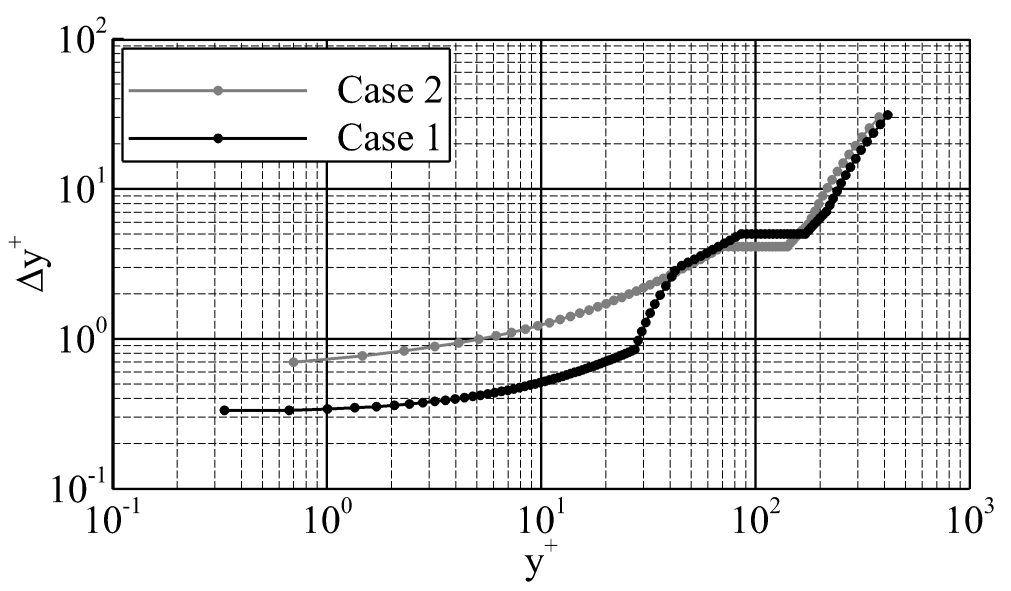

Figure 3.2: Wall-normal node distribution

$\Delta z^{+}=3$ in Case 2, to capture the smaller structures that develop in the interaction between the jet flow and the main flow. Fig. 3.2 presents the wall-normal node distribution below $y=22 \delta_{0}^{*}$. Above $y=22 \delta_{0}^{*}$, the node spacing is increased at a rate of $20 \%$ until the free-slip ceiling is reached. Outside of the thermal boundary layer, the wall-normal node distribution in Case 1 is closely based on the distribution used in Case 2 by Brinkerhoff and Yaras (2014) as observed in Fig. 3.2. Within the thermal boundary layer, the wall-normal node spacing in Case 1 is adjusted to accurately capture the smallest scale activities in the thermal field, which scale by $\operatorname{Pr}^{-1 / 3}$ (Redjem-Saad et al., 2007; Azih et al., 2012). After scaling, the first node from the wall is placed at $\mathrm{y}^{+}=0.33$ in Case 1 , whereas the first node is at $\mathrm{y}^{+}=0.70$ in Case 2. The node spacing values in Cases 1 and 2 are comparable to those used in the simulation of fully-turbulent boundary layers in literature, as discussed in section 3.4. Considering that the coherent structures of turbulence are typically of larger scale in young turbulent spots than in fully-turbulent boundary layers, the choice of node spacing in the present study is conservative. Finally, in the jet channel, node spacing in the $\mathrm{x}$ and $\mathrm{z}$ directions is set to $\Delta x^{+}=\Delta z^{+}=0.63$. This corresponds to at least 20 nodes in the boundary layer along the jet channel. In the streamwise direction in the jet channel, the node spacing is set to $\Delta y^{+}=4.8$, which is reduced at a rate of less 
Table 3.3: Number of nodes in Cases 1 and 2

\begin{tabular}{lccc}
\hline Computational domain & $N_{x}$ & $N_{y}$ & $N_{z}$ \\
\hline Case 1, Simulation 1 & 391 & 124 & 262 \\
Case 1, jet channel & 60 & 88 & 60 \\
Case 1, Simulation 2 & 374 & 127 & 262 \\
Case 2, Simulation 1 (Brinkerhoff and Yaras, 2014) & 345 & 82 & 266 \\
Case 2, jet channel (Brinkerhoff and Yaras, 2014) & 60 & 88 & 60 \\
Case 2, Simulation 2 (Brinkerhoff and Yaras, 2014) & 456 & 82 & 266 \\
\hline 2 excluding the jet channel & & &
\end{tabular}

than $15 \%$ to $\Delta y^{+}=0.80$ at the jet orifice plane. It was verified that the transition at the orifice plane from a node spacing of 0.80 to 0.33 , which is greater than the upper limit of $20 \%$ for the node spacing rate of change applied to the rest of the computational domain, does not affect the computed flow. Table 3.3 lists the node counts for Cases 1 and 2 .

To confirm that the results of the present study are grid independent, part of the growth of the turbulent spot in Simulation 2 of Case 1 was repeated with the number of nodes in the wall-normal, streamwise, and spanwise directions independently doubled in three simulations. Each simulation was solved for 1800 timesteps, which approximately corresponds to the length of time for one generation of hairpin vortices to form within a wave packet and to about $13 \%$ of the total simulated life of the spot. The turbulent spot and the vortical structures and streaks within the spot developed nearly identically in each of these simulations and in the original simulation. The change in the velocity in the region occupied by the turbulent spot between the simulations with the original grid and refined grids after 1800 timesteps is $1.5 \%$, $0.6 \%$, and $1.6 \%$ for grid refinement in the streamwise, wall-normal, and spanwise directions, respectively. These respectively correspond to $3.9 \%, 1.6 \%$, and $4.4 \%$ for the Richardson extrapolation fractional ordered error estimator (Roache, 1994). 
Working fluid: water

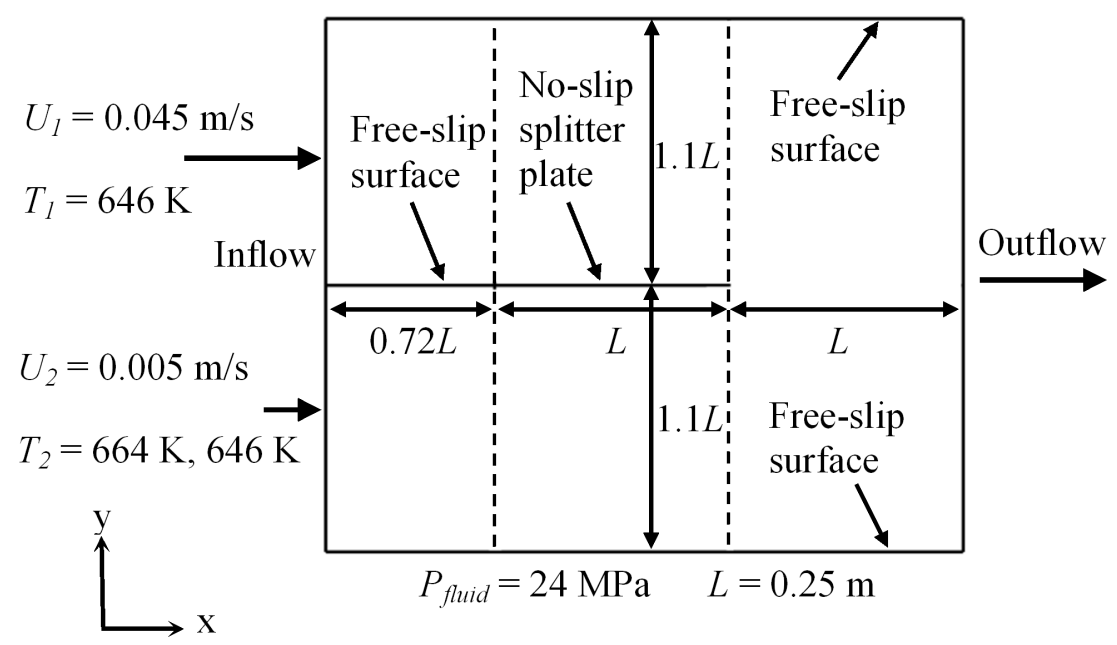

Figure 3.3: Computational domain for free shear layer instability simulations

\subsection{Free shear layer instability with cross-stream density and viscosity gradients}

Flow developments driven by the inviscid-unstable state of the shear layer enveloping low-speed streaks play a significant role in the regeneration of turbulence structures. The physical mechanisms by which this shear layer rolls up into hairpin vortices by the $\mathrm{K}-\mathrm{H}$ instability mechanism are fundamentally the same as in the roll-up of a free shear layer into K-H vortices. Thus, several two-dimensional direct numerical simulations of the roll-up of a free shear layer with large cross-stream density and viscosity gradients in the absence of buoyancy forces are performed to identify the isolated effects of these gradients on the growth of the $\mathrm{K}-\mathrm{H}$ instability in a more controlled setting than a flow field containing turbulence activity.

The computational domain used for these simulations is shown in Fig. 3.3. An infinitesimally-thin splitter plate separates low- and high-speed streams. The boundary layers developing on the two sides of the splitter plate merge downstream of the splitter plate forming a free shear layer. Since the initial roll-up of a free shear layer 
Table 3.4: Two-dimensional free shear layer simulations

\begin{tabular}{cccc}
\hline Case & Varied fluid properties & $T_{1}(\mathrm{~K})$ & $T_{2}(\mathrm{~K})$ \\
\hline Constant Properties & None & 646 & 646 \\
Variable Properties & $\rho, \mu$ & 646 & 664 \\
Variable Density & $\rho$ & 646 & 664 \\
Variable Viscosity & $\mu$ & 646 & 664 \\
\hline
\end{tabular}

is a two-dimensional phenomenon, for computational efficiency a two-dimensional computational domain of $0.0008 \mathrm{~L}$ width bound by side boundaries with periodic conditions is used. The top and bottom surfaces of the computational domain are conservatively placed far from the splitter-plate surface so that they do not influence the development of the free shear layer.

A list of the test cases is presented in Table 3.4. The cross-stream temperature and velocity gradients are of opposite sign, which is consistent with the state of a boundary layer developing over a heated wall. The thermal conductivity and specific heat capacity are allowed to vary with temperature in the Variable Properties, Variable Density, and Variable Viscosity cases. The effects of spatial variations in these properties on flow instability have been established to be several orders of magnitude lower than those of density and viscosity (Azih and Yaras, 2013; Wazzan et al., 1972; Schäfer et al., 1995; Özgen, 2004).

Uniform inflow velocity and temperature values are specified for each of the two streams as shown in Fig. 3.3. The streamwise distance downstream of the splitterplate trailing edge at which the roll-up of the free shear layer is completed is dependent on the disturbance amplification rate, which increases linearly with the velocity ratio (Monkewitz and Huerre, 1982). A velocity ratio of $R=\left(U_{1}-U_{2}\right) /\left(U_{1}+U_{2}\right)=0.8$ is used to ensure that the free shear layer rolls up between $x / L=0.15$ and $x / L=0.35$ downstream of the splitter-plate trailing edge, well upstream of the outflow boundary. The inflow velocity of the high-speed stream is such that the Reynolds number at the 
trailing edge of the splitter plate is $\operatorname{Re}_{\theta}=185$, which is below the critical Reynolds number of $\operatorname{Re}_{\theta_{\text {crit }}}=200$ predicted by linear stability theory. This ensures that the boundary layer on the splitter plate remains fully laminar. The outflow boundary is set to a fixed area-averaged pressure value of $24 \mathrm{MPa}$.

In the cases with variations in density and/or viscosity, the splitter-plate surface temperature is varied linearly between the respective high- and low-speed stream inlet temperatures stated in Table 3.4 at the no-slip splitter-plate leading edge and a trailing edge temperature of $655 \mathrm{~K}$. These temperature boundary conditions yield cross-stream property gradients in the wake of the splitter plate which are larger than those prevailing in the turbulent spot simulations, where the density gradients are kept at lower levels to prevent the spot from growing too rapidly. An adiabatic thermal boundary condition is specified for the free-slip surface upstream of the splitter plate and the top and bottom surfaces of the computational domain. In each simulation, the upper and lower halves of the computational domain are initialized with the corresponding inflow temperature and velocity values.

The boundary layer thickness at the splitter-plate trailing edge in the high-speed stream is about $95 \%$ of the undisturbed boundary layer thickness downstream of the jet orifice in the Case 1 turbulent spot simulation. Accordingly, the wall-normal node distribution above and below the splitter plate is set to be the same as that shown in Fig. 3.2. A streamwise node spacing of $\Delta x^{+}=15$ is used throughout the domain, except for the region above and below the free-slip surface upstream of the splitter plate where it is increased at a rate of $10 \%$ until the inflow boundary is reached. All node spacing values are normalized based on the viscous length scale computed at the splitter-plate trailing edge in the high-speed stream. 


\subsection{Solution method}

The commercial software package ANSYS CFX 13.0 is used to solve the continuity, momentum, and energy equations. The author's research group has used this software extensively in direct numerical simulations of attached and separated shear layers in transitioning and turbulent states (Azih et al., 2012; Brinkerhoff and Yaras, 2012; John, 2012; Brinkerhoff and Yaras, 2014). Property tables from the IAPWS-IF97 database are used to obtain the thermophysical properties for water at supercritical conditions. The governing equations are spatially discretized using a finite volume approach. Spatial derivatives are evaluated using second-order centred-differencing, and second-order Euler backward-differencing is used for temporal derivatives. Benchmark simulations performed by the author's research group have shown a root-meansquare normalized residual value on the order of $10^{-6}$ to be adequate for satisfactory

convergence. Convergence of the equations to this residual value is realized through outer-loop iterations at each timestep which converge the coefficients of the linearized equations and one inner-loop iteration per outer-loop iteration in which the linearized equations are solved with W-type algebraic multigrid cycles. The multigrid cycle uses six grid levels, with respectively one and three solution sweeps performed at each grid level during the restriction and prolongation passes of each cycle.

In the simulation of the turbulent spot, the undisturbed laminar boundary layer in each of the two parts of the simulation is developed by solving for the steady-state solution of the discretized equations. This is performed by integrating the governing equations in time with one outer-loop iteration per timestep. The coefficients of the governing equations are not converged within each timestep, which implies the temporal development of the laminar boundary layer is not physically realistic. Rather, the timestep is adjusted to serve as a means of under-relaxation, and larger timesteps are used to accelerate the convergence of the steady-state solution. Once 
the laminar boundary layer is developed, the solution of the temporal development of the jet-main-flow interaction and the growth of the turbulent spot is obtained by integrating the equations in time with a timestep size of $7.86 \times 10^{-4} s$ in Case 1 using up to six outer-loop iterations per timestep. This corresponds to $\Delta t^{+}=0.045$, which is an order of magnitude smaller than the value of $\Delta t^{+}=0.2$ recommended to satisfactorily capture the temporal growth of turbulence structures (Choi and Moin, 1994). In Case 2, timestep sizes of $7.0 \times 10^{-6} s$ and $3.5 \times 10^{-5} s$ are used in the first and second simulations, respectively, which correspond to a maximum normalized timestep of $\Delta t^{+}=0.11$. Simulation 1 of Case 1 was performed using 20 AMD Opteron 6128 CPU cores and took approximately 64,000 CPU hours. Simulation 2 of Case 1 was performed using 24 Intel Xeon L5410 CPU cores and took approximately 41,800 CPU hours. Brinkerhoff and Yaras (2014) used 20 Intel Xeon L5410 CPU cores for Simulations 1 and 2 of Case 2, which took about 2000 and 3600 CPU hours, respectively.

In the two-dimensional simulations of free shear layer instability, the laminar boundary layer on each side of the splitter plate and the free shear layer downstream of the splitter plate are first developed using one outer-loop iteration per timestep and timesteps that are large enough $\left(\Delta t^{+}>60\right)$ to fully dampen any disturbances in the free shear layer such that temporal and spatial instabilities do not develop anywhere in the flow. This initial flow field is then developed in a time-accurate manner by using up to six outer-loop iterations per timestep and a timestep of $0.0025 \mathrm{~s}$, which corresponds to $\Delta t^{+}=0.075$. Flow-field data for analysis is collected once changes in the vortex shedding frequency drop below 5\%. Each simulation was performed using 4 AMD Opteron 6128 CPU cores. The Constant Properties, Variable Properties, Variable Density, and Variable Viscosity simulations took approximately 400, 2,160, 1,840, and 520 CPU hours, respectively. 
Table 3.5: Node distributions used in direct numerical simulation studies of a turbulent boundary layer

\begin{tabular}{lcccc}
\hline Simulation & $\Delta x^{+}$ & $\Delta z^{+}$ & $\Delta y_{\text {wall }}^{+}$ & $\operatorname{Re}_{\theta}$ \\
\hline Grid 1 & 3 & 3 & 0.85 & 500 \\
Grid 2 & 22 & 11 & 0.85 & 500 \\
Spalart (1988) & 20 & 7 & 0.80 & 670,1410 \\
\hline
\end{tabular}

\subsection{Validation of the numerical approach}

Validation of the spatial grid, time-step sizing, and the numerical algorithm was realized by the author's research group through direct numerical simulations of an incompressible, zero-pressure-gradient, fully-turbulent boundary layer developing on a flat plate (Brinkerhoff and Yaras, 2011b; Azih et al., 2012). The results were compared to the direct numerical simulations by Spalart (1988), who used a spectral method for spatial discretization and a second-order hybrid Runge-Kutta/Crank-Nicolson scheme for temporal discretization, and to the experimental results of Djenidi and Antonia (1993). Table 3.5 presents the node spacing values of these simulations, which are similar to those used in Cases 1 and 2 shown in Table 3.2.

Fig. 3.4 shows that the rates of turbulence production $\left(P^{+}\right)$and dissipation $\left(\epsilon^{+}\right)$and the mean velocity profile of these validation simulations compare well with published results, with only slight discrepancies compared to the turbulence kinetic energy budgets computed by Spalart (1988) and the mean velocity profile measured by Djenidi and Antonia (1993). The differences in the results observed in Fig. 3.4a are similar to the uncertainty ranges in the turbulence production and dissipation budgets in published literature (Honkan and Andreopoulos, 1997). 

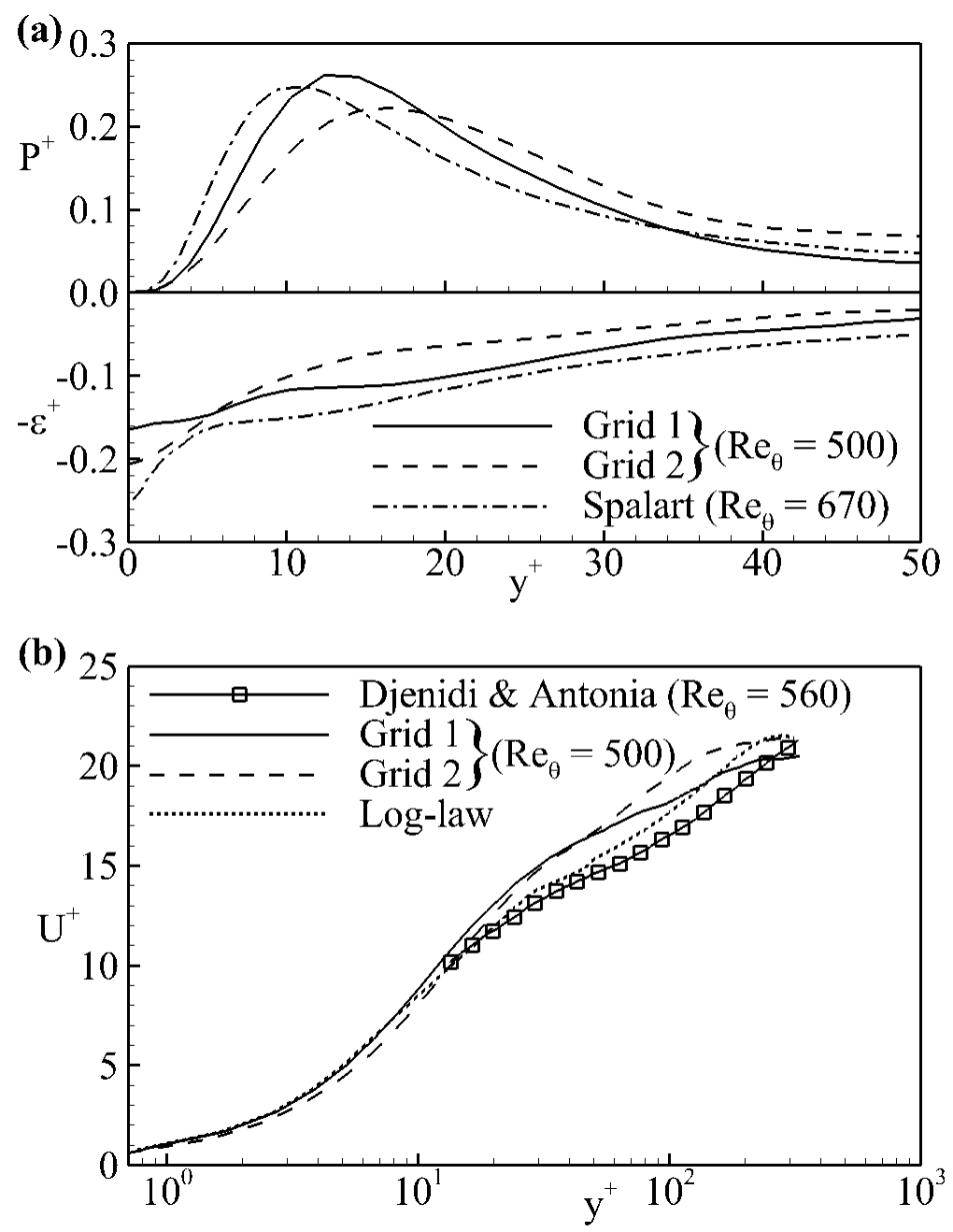

Figure 3.4: Computed (a) turbulence production and dissipation rates, and (b) mean velocity profile. Adapted from Brinkerhoff and Yaras (2011b). 


\section{Chapter 4}

\section{Results and Discussion}

\subsection{Undisturbed laminar flow field in the turbulent spot sim- ulations}

The streamwise distributions of the freestream velocity, the undisturbed boundary layer displacement thickness, and the streamwise acceleration parameter in Case 1 are presented in Fig. 4.1. The acceleration parameter is given by:

$$
\eta=\frac{\nu}{U_{\infty}^{2}} \frac{d U_{\infty}}{d x}
$$

The kinematic viscosity, $\nu$, in Eq. (4.1) is evaluated using a spatial average in the wall-normal direction over the local velocity boundary layer. The local freestream velocity is denoted by $U_{\infty}$. The value of the acceleration parameter downstream of the jet orifice where the turbulent spot develops is within the range of $\eta$ values, $\eta_{\text {crit }}$, at which a turbulent boundary layer is expected to re-laminarize in a flow with uniform thermophysical properties (Escudier et al., 1998; Jones and Launder, 1972; Moretti and Kays, 1965).

Fig. 4.2 and 4.3 present the wall-normal distributions of thermophysical properties and their gradients in the undisturbed laminar boundary layer at the streamwise 


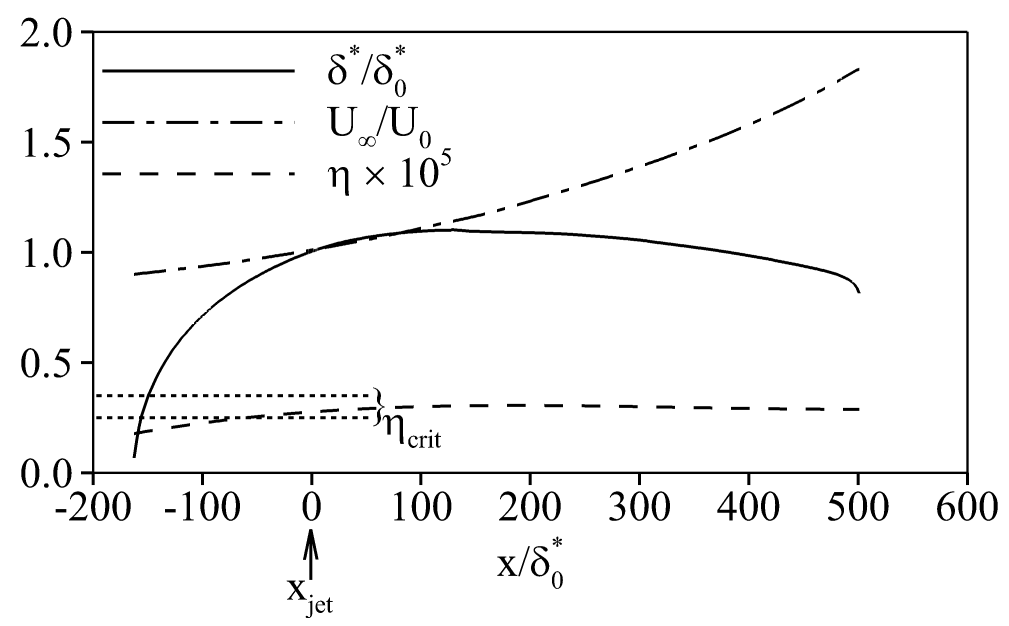

Figure 4.1: Streamwise distributions of the freestream velocity, undisturbed boundary layer displacement thickness, and acceleration parameter in Case 1

location of the jet orifice in Case 1. The velocity profile at this location is shown in Fig. 4.4. The velocity profile in Case 1 is significantly fuller than in Case 2, which is due to the favourable streamwise pressure gradient in Case 1. There is no evidence of pre-transition activity in the form of streaky structures in the boundary layer. The maximum fluctuations in streamwise velocity in the laminar boundary layer do not exceed $0.2 \%$ of $U_{0}$.

The Reynolds number of Case 1 based on the freestream velocity and momentum thickness spatially-averaged over the footprint of the turbulent spot and the kinematic viscosity spatially-averaged over the local velocity boundary layer thickness within the turbulent spot is about $\operatorname{Re}_{\theta}=150$ at the end of Simulation 1 and $\operatorname{Re}_{\theta}=160$ at the end of Simulation 2. The physical mechanisms which govern the regeneration of turbulence structures within a turbulent spot are fundamentally the same as those within a turbulent boundary layer at the same Reynolds number; however, the organization of turbulence structures within a turbulent spot is typically more orderly (Schröder and Kompenhans, 2004). This is due to the presence of a broader range of generations of mutually-interacting wave packets in the instance of a fully-developed turbulent boundary layer. Hence, the physical mechanisms by which the wall-normal gradients 


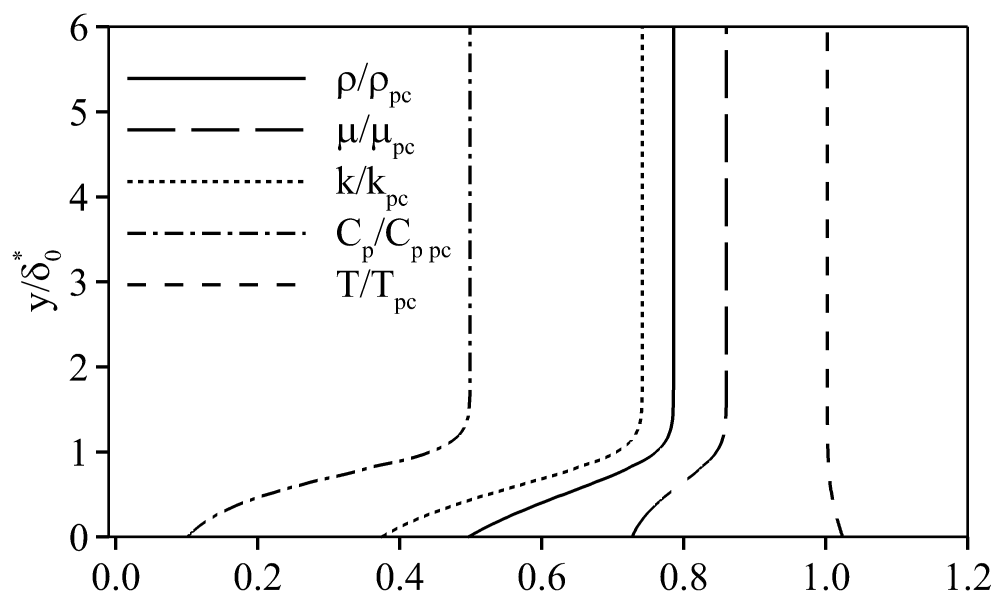

Figure 4.2: Undisturbed wall-normal profiles of density, viscosity, thermal conductivity, isobaric specific heat capacity, and temperature in Case 1 at the streamwise location of the jet orifice

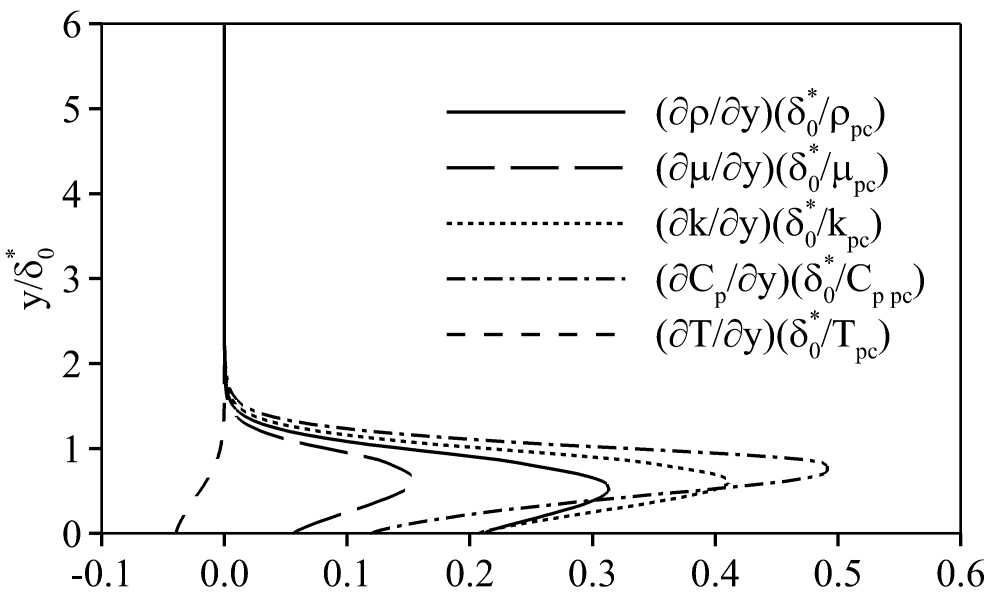

Figure 4.3: Undisturbed wall-normal profiles of gradients in density, viscosity, thermal conductivity, isobaric specific heat capacity, and temperature in Case 1 at the streamwise location of the jet orifice 


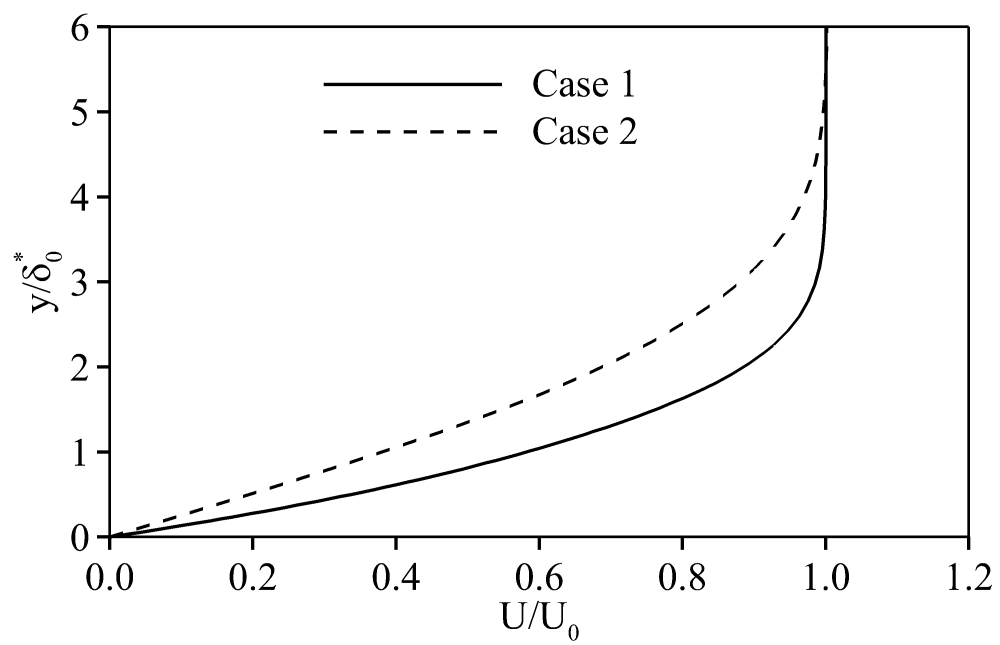

Figure 4.4: Profiles of undisturbed streamwise velocity in Cases 1 and 2 at the streamwise location of the jet orifice

in density are observed to alter the regeneration of turbulence structures within the spot are expected to be fundamentally the same as those which would occur within a fully-turbulent boundary layer at this Reynolds number. The inlet flow Reynolds number of supercritical water coolant in the preliminary design of next generation nuclear reactors is estimated to be $\operatorname{Re}_{\delta} \sim O\left(10^{5}\right)$, which corresponds to $\operatorname{Re}_{\theta} \sim O\left(10^{4}\right)$ (Duffey and Pioro, 2005; Azih et al., 2012). Experimental flow visualization studies performed up to these Reynolds numbers have shown that the fundamental characteristics of turbulence structures, wave packets, and low-speed streaks experience only small variations between Reynolds number values of this magnitude and the Reynolds number values in the simulations of the present study (Adrian et al., 2000; Smith and Metzler, 1983; Head and Bandyopadhyay, 1981). Streamwise and spanwise spacings between hairpin vortices in wave packets and spanwise spacing between low-speed streaks remain consistent over the Reynolds number range considered when scaled on viscous wall variables (Adrian et al., 2000; Smith and Metzler, 1983). Boundary layer flows at higher values of Reynolds number contain a greater number of hairpin 


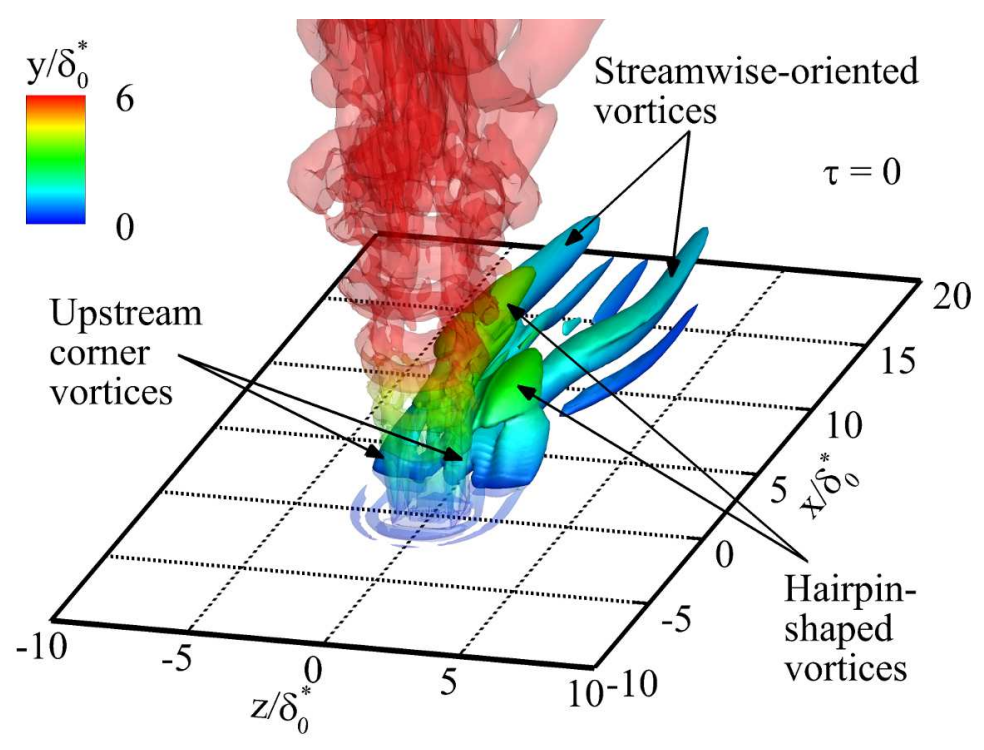

Figure 4.5: Visualization of the jet-main-flow interaction in Case 1 at the last timestep of the jet pulse using iso-surfaces of $Q=2 \times 10^{-2}$

vortices in wave packets, and a greater range of generations of wave packets of differing sizes and convection speeds (Adrian et al., 2000; Tomkins and Adrian, 2003). Consequently, mutual interactions between structures are generally more complex and more frequent at higher Reynolds number values (Tomkins and Adrian, 2003). Despite such differences, it is expected that the fundamental mechanisms by which large wall-normal gradients in density affect the development of turbulence structures observed in this study will still be present at higher Reynolds number values.

\subsection{Turbulent spot development}

The interaction between the pulsed jet and the main-flow laminar boundary layer at the last timestep of the jet pulse in Case 1 is shown in Fig. 4.5. The vortical structures in the jet column are shown translucently to better visualize the structures forming in the boundary layer immediately downstream of the jet orifice from which the turbulent spot develops. The ranges of the $\mathrm{x}$ - and $\mathrm{z}$-axis are chosen to show all relevant flow structures within the much-larger computational domain. The vortical 
structures are visualized using iso-surfaces of the second invariant of the velocitygradient tensor normalized by $\left(U_{0} / \delta_{0}^{*}\right)^{2}$, denoted $Q$. $Q$ is defined as:

$$
Q=\frac{1}{2}\left(\frac{\partial u_{i}}{\partial x_{i}} \frac{\partial u_{j}}{\partial x_{j}}-\frac{\partial u_{i}}{\partial x_{j}} \frac{\partial u_{j}}{\partial x_{i}}\right)
$$

where $u_{i}$ and $u_{j}$ represent the components of the perturbation velocity vector, and Einstein summation is applied over the indexed terms. Perturbation quantities in the present study are defined as the difference between the instantaneous and undisturbed flow fields. Further information on $Q$ is provided in Appendix B. The value of $Q$ at which the iso-surfaces are plotted is chosen to most clearly show all vortical structures which affect the dynamics of the flow.

The vortical structures associated with the jet-main-flow interaction largely originate from the vorticity in the jet channel, since the vorticity in the jet channel laminar boundary layer is two orders of magnitude greater than the vorticity in the main-flow laminar boundary layer. The wall-normal gradients in thermophysical properties in Case 1 are only present in the main-flow boundary layer; the jet channel flow is at a uniform temperature. Consequently, the density gradients have a negligible effect on the jet-main-flow interaction in Case 1 so that it develops in a similar manner as in Case 2. Vertically-oriented vortices roll up in the jet flow near the corners of the jet orifice immediately above the orifice. The two vortices which form near the upstream corners of the jet orifice are labelled in Fig. 4.5; vortices also form near the downstream corners. Spanwise-oriented vorticity originating in the jet channel boundary layer along the downstream jet channel wall, upon being ejected from the square orifice, is re-oriented in the streamwise direction by the velocity field induced by the corner vortices (Brinkerhoff and Yaras, 2012). These counter-rotating streamwiseoriented vortices grow in the streamwise direction downstream of the orifice over the duration of the jet pulse. They lift the spanwise vorticity in the main-flow boundary 
layer away from the wall immediately downstream of the jet resulting in the formation of one hairpin-shaped vortex straddling each streamwise-oriented vortex. The legs of these hairpin vortices extend to the wall on either side of the corresponding streamwise vortex. This jet-main-flow interaction observed in Case 1 follows the same pattern of development noted in Case 2 (Brinkerhoff and Yaras, 2014).

The two initial hairpin vortices grow in size as they convect downstream, eventually merging to form one larger hairpin vortex, which is the primary hairpin vortex of the turbulent spot identified as structure 'A' in Fig. 4.6a. This is followed by the development of several secondary hairpin vortices upstream of the primary hairpin vortex. Hairpin vortices labelled with the same letter form in close proximity to each other at a similar time, and are numbered according to the temporal order of formation. The smaller secondary hairpin vortices merge together into larger hairpin vortices by $\tau=175$, as labelled in Fig. 4.6b; for example, B1 and B2 merge into hairpin vortex B1-B2. This is followed by the formation of tertiary hairpin vortices upstream and downstream of these secondary hairpin vortices, such that the spot is soon densely populated with coherent structures of turbulence.

The development of hairpin vortices within the Case- 1 spot in this manner is very similar to the evolution of hairpin vortex topology in Case 2 described by Brinkerhoff and Yaras (2014). The ejection of low-speed fluid by the wall-normal motions induced by the legs of existing parent hairpin vortices leads to the development of low-speed streaks between their legs. An inflectional streamwise velocity profile develops at the interface between the low-speed streaks and the surrounding higher-speed flow. Streamwise grouping of vorticity within this shear layer by the K-H instability mechanism leads to the roll-up of this shear layer into new hairpin vortices. Smith et al. (1991) and Singer and Joslin (1994) suggest that new hairpin vortices have a tendency to develop between the legs of a parent hairpin vortex, while Schröder and Kompenhans (2004) and Schröder et al. (2008) observed new hairpin vortices 
(a) $\tau=118$

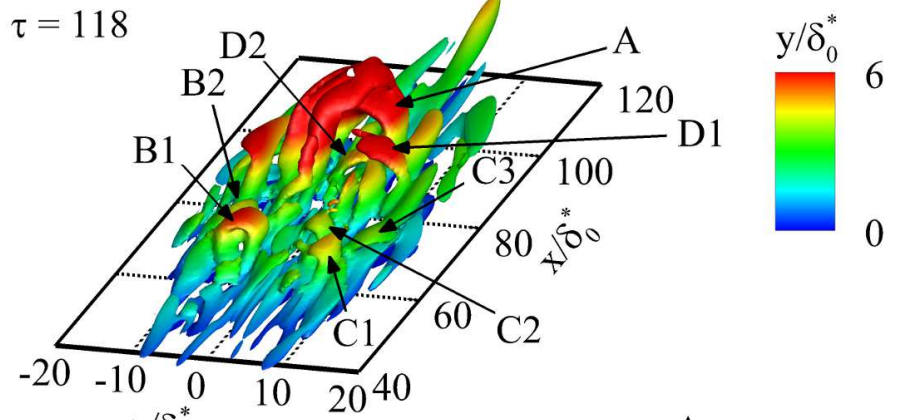

(b) $\tau=175$
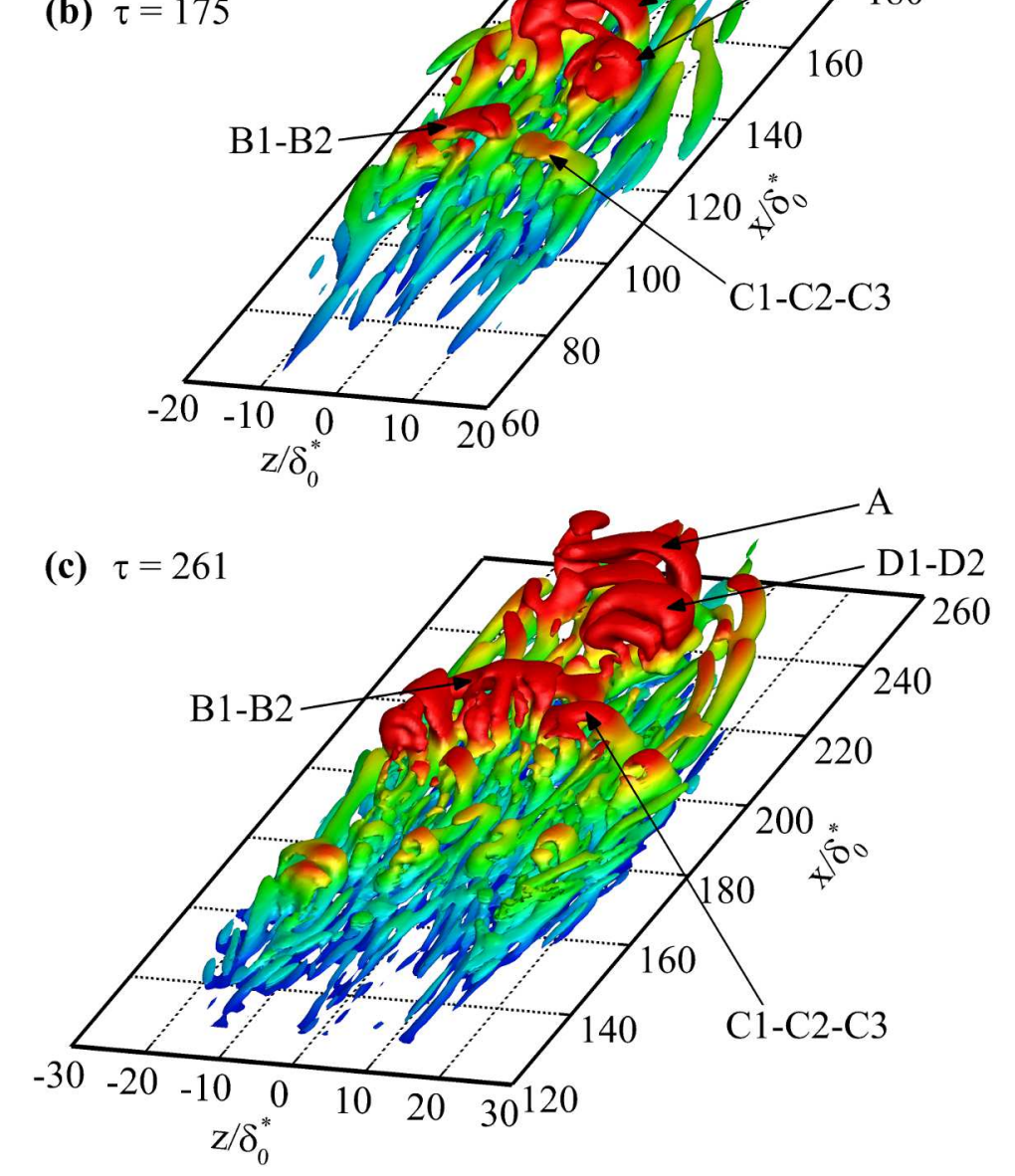

Figure 4.6: Visualization of hairpin vortices during the early development of the spot in Case 1 using iso-surfaces of $Q=2 \times 10^{-4}$ 

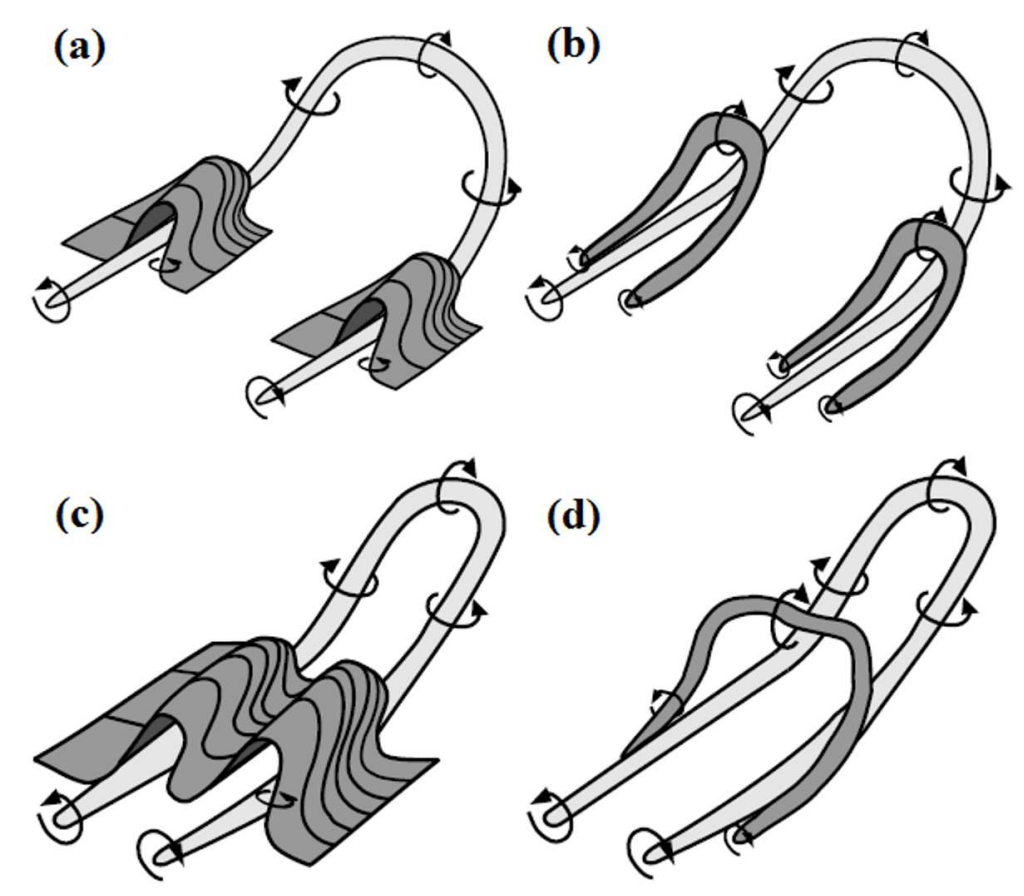

Figure 4.7: Illustration of the development of new hairpin vortices from a parent hairpin vortex with (a)-(b) widely-spaced legs and (c)-(d) closely-spaced legs; adapted from Brinkerhoff and Yaras (2014)

which straddle the legs of the parent hairpin vortex. Brinkerhoff and Yaras (2014) showed that the same fundamental physical mechanisms occur in both scenarios. When the legs of the parent hairpin vortex are widely-spaced, a low-speed streak develops next to each leg. The shear layer enveloping each low-speed streak rolls up into a separate hairpin vortex, as shown in Fig. 4.7a and b (Brinkerhoff and Yaras, 2014). On the other hand, when the legs of the parent hairpin vortex are closely-spaced, the regions of low-speed flow that are lifted away from the wall next to each leg merge together to form a single region of low-speed fluid. The shear layer enveloping this region evolves into a single hairpin vortex, as shown in Fig. 4.7c and d (Brinkerhoff and Yaras, 2014). The first mechanism is typically observed among the large-scale hairpin vortices near the leading edge and spanwise edges of the spot, and the second mechanism is typically observed among smaller-scale hairpin vortices near the trailing 

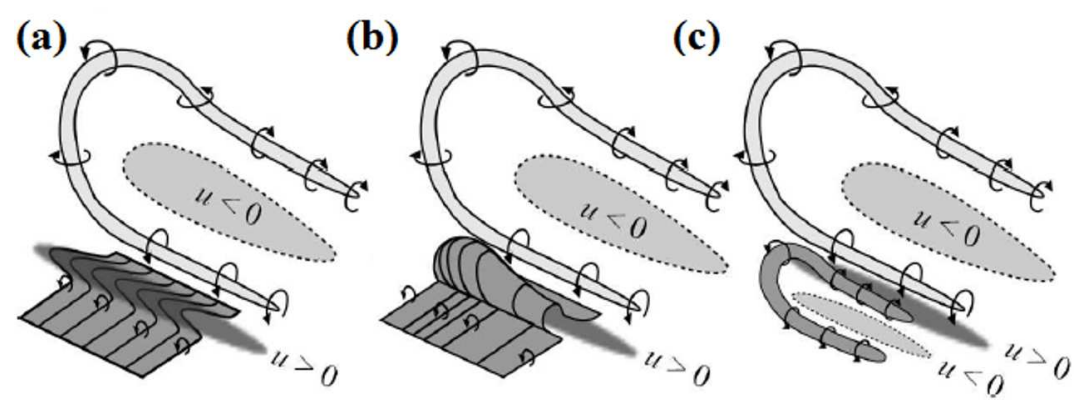

Figure 4.8: Illustration of the lateral spreading of the spot by the generation of hairpin vortices along the spot spanwise edges; adapted from Brinkerhoff and Yaras (2014)

edge of the spot (Brinkerhoff and Yaras, 2014).

Brinkerhoff and Yaras (2014) demonstrated that lateral growth of the spot occurs through generation of new wave packets along the spot spanwise edges, and follows the same fundamental processes as in the formation of hairpin vortices within the interior of the spot. This is illustrated schematically in Fig. 4.8. In the figure, $u$ denotes the streamwise component of perturbation velocity. Downwash motions on the outboard sides of the legs of hairpin vortices which reside along the spanwise edges of the spot perturb the laminar flow beyond the laminar/turbulent interface on the sides of the spot (Brinkerhoff and Yaras, 2014). The high-speed flow which is swept toward the wall stretches the background spanwise vorticity in the streamwise direction creating streamwise-oriented vorticity which lifts itself away from the wall by self-induced motions (Brinkerhoff and Yaras, 2014). The resulting shear layer rolls up into new hairpin vortices, which induce wall-normal motions to form new highand low-speed streaks along the spot spanwise edges.

The turbulent spots developing in Cases 1 and 2 are compared at the same instance in time in Fig. 4.9. Although the fundamental nature of the flow development in the spot is the same in each case, the Case- 1 spot contains more densely-spaced hairpin vortices and is of a larger size, despite being exposed to a strong favourable streamwise pressure gradient. The results presented in section 4.4 show this trend to be primarily 


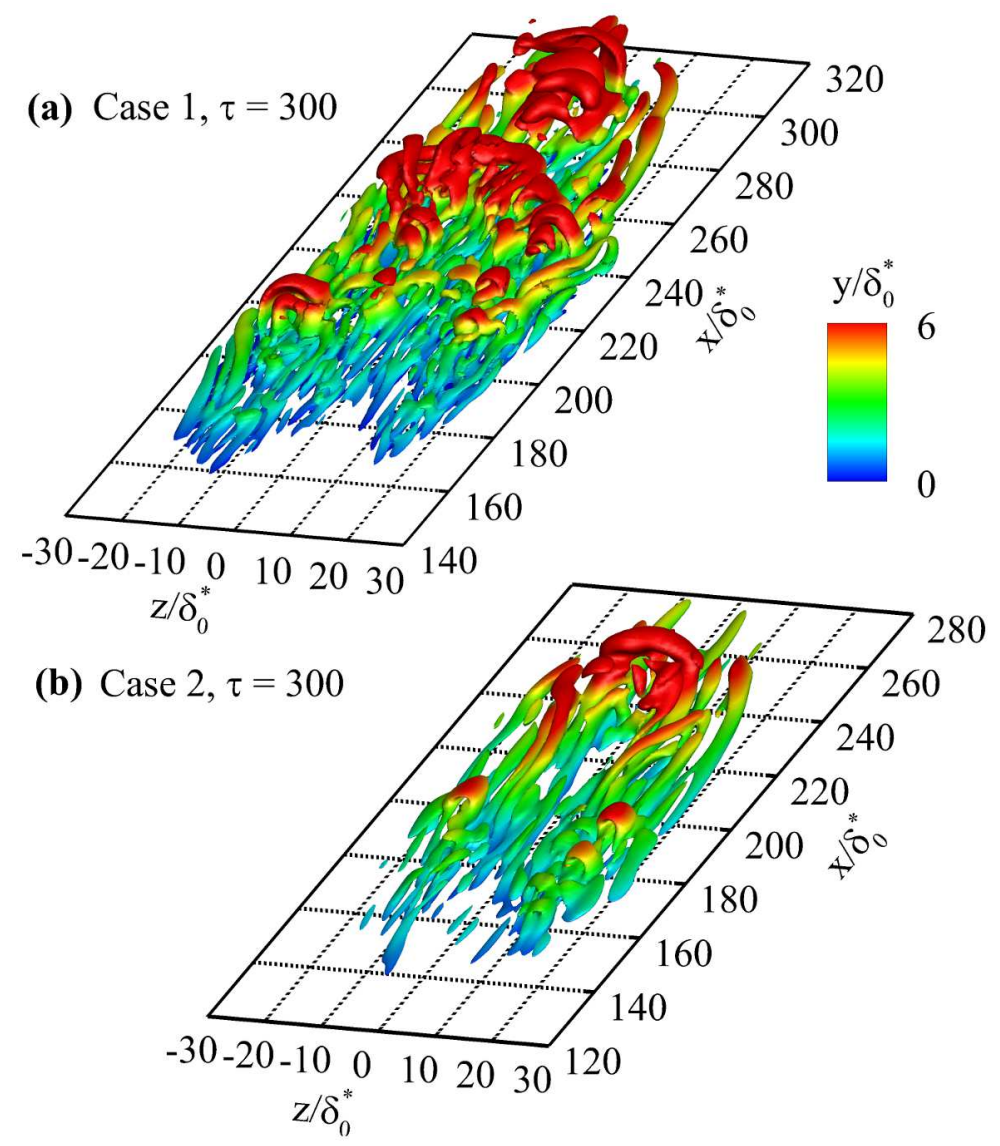

Figure 4.9: Visualization of the turbulent spots in Cases 1 and 2 using iso-surfaces of $Q=2 \times 10^{-4}$

the result of spatial gradients in density. The mechanisms by which these gradients cause this increased concentration of hairpin vortices within the turbulent spot is discussed in the following sections.

\subsection{Effects of spatial density gradients on the formation of hairpin vortices and their mutual interactions}

Fig. 4.10 and 4.11 show the formation of two different hairpin vortices during the early growth of the Case-1 spot. A time period early in the life of the spot is chosen because only a few hairpin vortices are present, and their formation is most clearly 

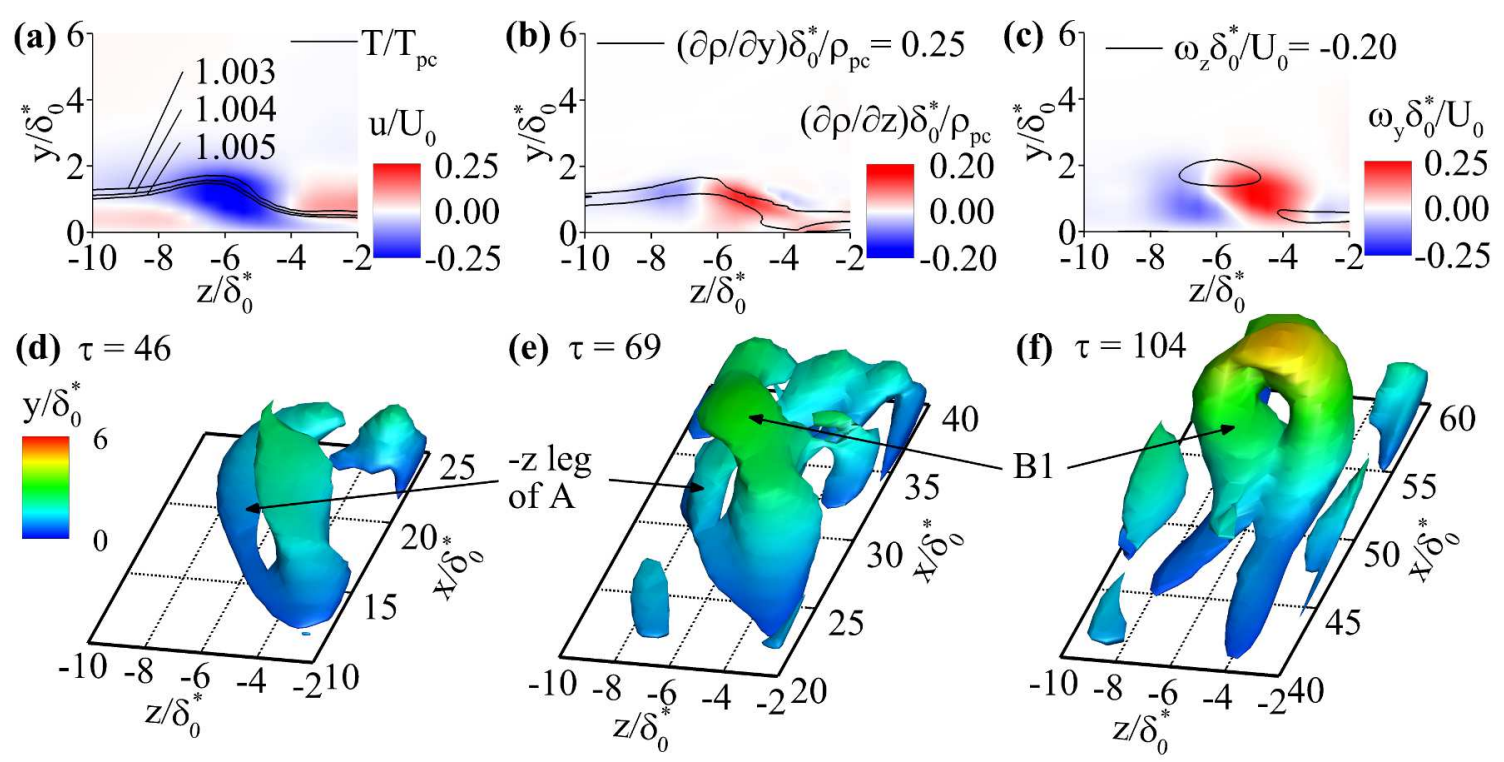

Figure 4.10: Formation of the B1 hairpin vortex in Case 1. (a)-(c): yz planes at $x / \delta_{0}^{*}=19, \tau=46$ of (a) temperature and streamwise component of perturbation velocity (u), (b) density gradients in the wall-normal and spanwise directions, and (c) components of perturbation vorticity $(\omega)$ in the wall-normal and spanwise directions. (d)-(f): iso-surfaces of $Q=2 \times 10^{-3}$ at (d) $\tau=46$, (e) $\tau=69$, and (f) $\tau=104$.
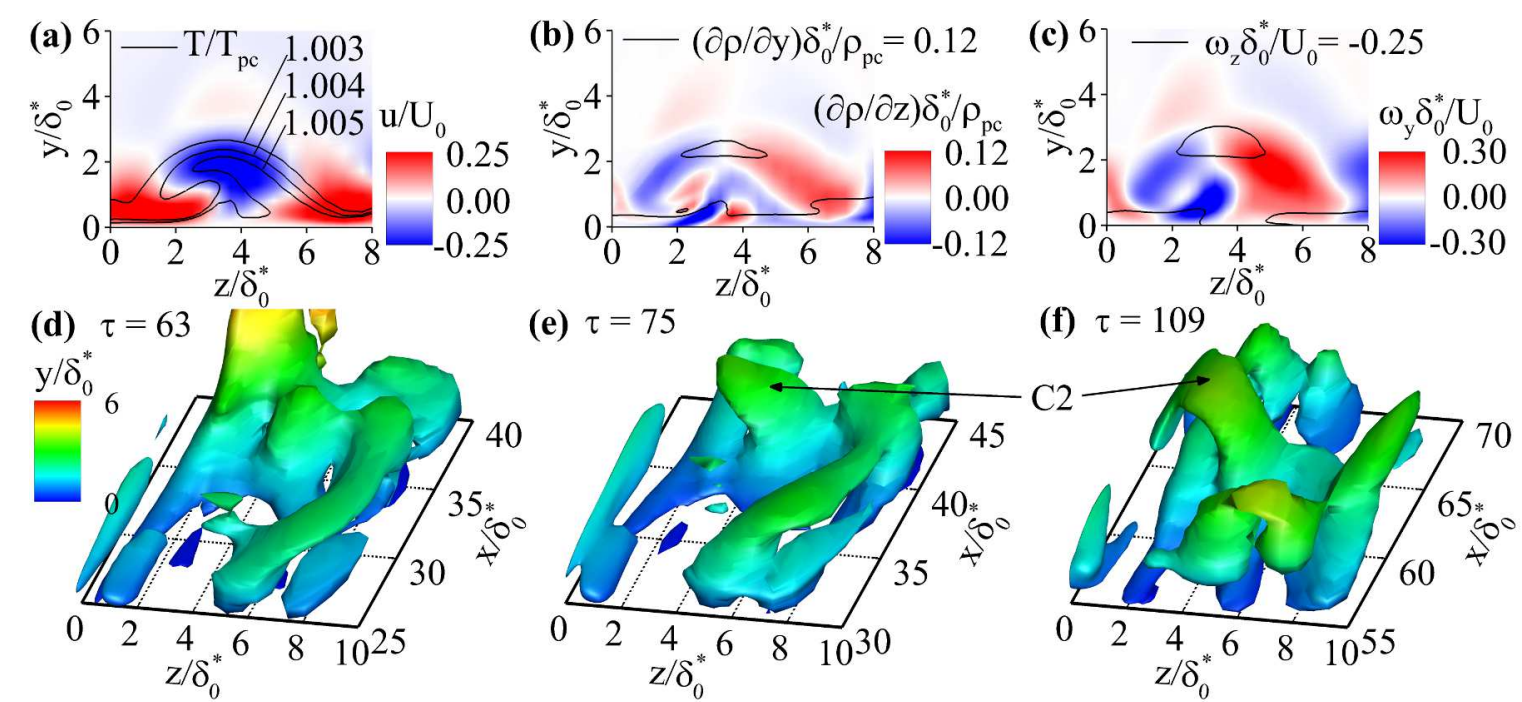

(f) $\tau=109$

Figure 4.11: Formation of the $\mathrm{C} 2$ hairpin vortex in Case 1. (a)-(c): yz planes at $x / \delta_{0}^{*}=41, \tau=75$ of (a) temperature and streamwise component of perturbation velocity (u), (b) density gradients in the wall-normal and spanwise directions, and (c) components of perturbation vorticity $(\omega)$ in the wall-normal and spanwise directions. (d)-(f): iso-surfaces of $Q=2 \times 10^{-3}$ at (d) $\tau=63$, (e) $\tau=75$, and (f) $\tau=109$. 
defined. However, the spot has travelled sufficiently far downstream such that it is no longer affected by the disturbance used to trigger the spot, and the mechanisms by which the turbulence structures develop are representative of those that prevail when the spot reaches a more mature state.

Fig. 4.10a shows a cross section in the yz plane of the low-speed streak which lies next to the $-\mathrm{z}$ leg of primary hairpin vortex A. Ejected lower-speed fluid is of higher temperature than the fluid away from the wall, such that the low-speed streak is at a slightly higher temperature than the local surrounding fluid. Likewise, higher-speed fluid which is swept toward the wall is at a lower temperature than the fluid near the wall. Consequently, temperature gradients develop along the perimeter of the low-speed streak, which are shown by the line contours in Fig. 4.10a. Fig. 4.10b shows that substantial density gradients develop along the sides and top of the streak because the temperature of the fluid is close to the pseudocritical temperature. Since the viscosity gradients follow a nearly identical spatial distribution as the density gradients and the effects of the gradients in viscosity are later shown to be negligible, only the density gradients are presented. The viscosity gradients, when normalized by $\delta_{0}^{*}$ and $\mu_{p c}$, are of a maximum magnitude of about 0.1 in the formation of hairpin vortex B1 in Fig. 4.10, and about 0.05 in the formation of hairpin vortex $\mathrm{C} 2$ in Fig. 4.11. Fig. 4.10c shows the vorticity field associated with the inviscid-unstable shear layer enveloping the low-speed streak. The density spatial gradients are largest in the regions occupied by this shear layer, which rolls up into hairpin vortex B1 by the $\mathrm{K}-\mathrm{H}$ instability mechanism as shown in Fig. 4.10e and $\mathrm{f}$. This hairpin vortex continues to grow in size as it convects downstream. The development of hairpin vortex $\mathrm{C} 2$ in Fig. 4.11 occurs by the same mechanisms as described for B1.

The spot in Case 1 contains more hairpin vortices than in Case 2, and the spatial gradients in density are largest in the regions occupied by the shear layer enveloping 
the low-speed streaks, as shown in Fig. 4.10 and 4.11. This suggests that the density gradients likely promote the K-H instability mechanism in this shear layer, thus promoting the roll-up of this shear layer into hairpin vortices.

The roll-up of the shear layer enveloping the low-speed streaks through the $\mathrm{K}-\mathrm{H}$ mechanism is also responsible for the deformation of the streaks along their length. This deformation develops as per two distinct patterns, identified as sinuous and varicose instability modes (Swearingen and Blackwelder, 1987; Reddy et al., 1998; Elofsson et al., 1999). The sinuous mode is characterized by oscillations in the streaks in the streamwise-spanwise plane, whereas the varicose mode occurs in the form of a streamwise-periodic widening and narrowing of the streaks. The sinuous mode is primarily driven by the inviscid instability of the shear layer along the sides of the streaks, and the varicose mode is primarily driven by the inviscid instability of the shear layer above the streaks (Swearingen and Blackwelder, 1987; Reddy et al., 1998; Elofsson et al., 1999). The sinuous mode is more unstable than the varicose mode and occurs at a lower threshold of streamwise perturbation velocity magnitude in the streak (Swearingen and Blackwelder, 1987; Reddy et al., 1998; Elofsson et al., 1999). Fig. 4.12 compares the streaks of Cases 1 and 2 when the spots are of similar size, and shows the streaks in Case 1 to be more unstable by the sinuous instability mode than in Case 2. Specifically, the streaks in Case 2 remain stable in the downstream half of the spot, and exhibit instability only in the upstream half of the spot. Conversely, the streaks in Case 1 are unstable throughout the entire spot, despite the strong stabilizing effect of the favourable streamwise pressure gradient. The spanwise oscillations in the streaks due to the enhanced sinuous instability mode in Case 1 result in closer spanwise proximity of adjacent low-speed streaks at selective locations along their lengths.

The spatial distribution of the spanwise density gradients within the Case- 1 spot is shown in Fig. 4.13. The streaks in the Case- 1 spot are likely more unstable by the 

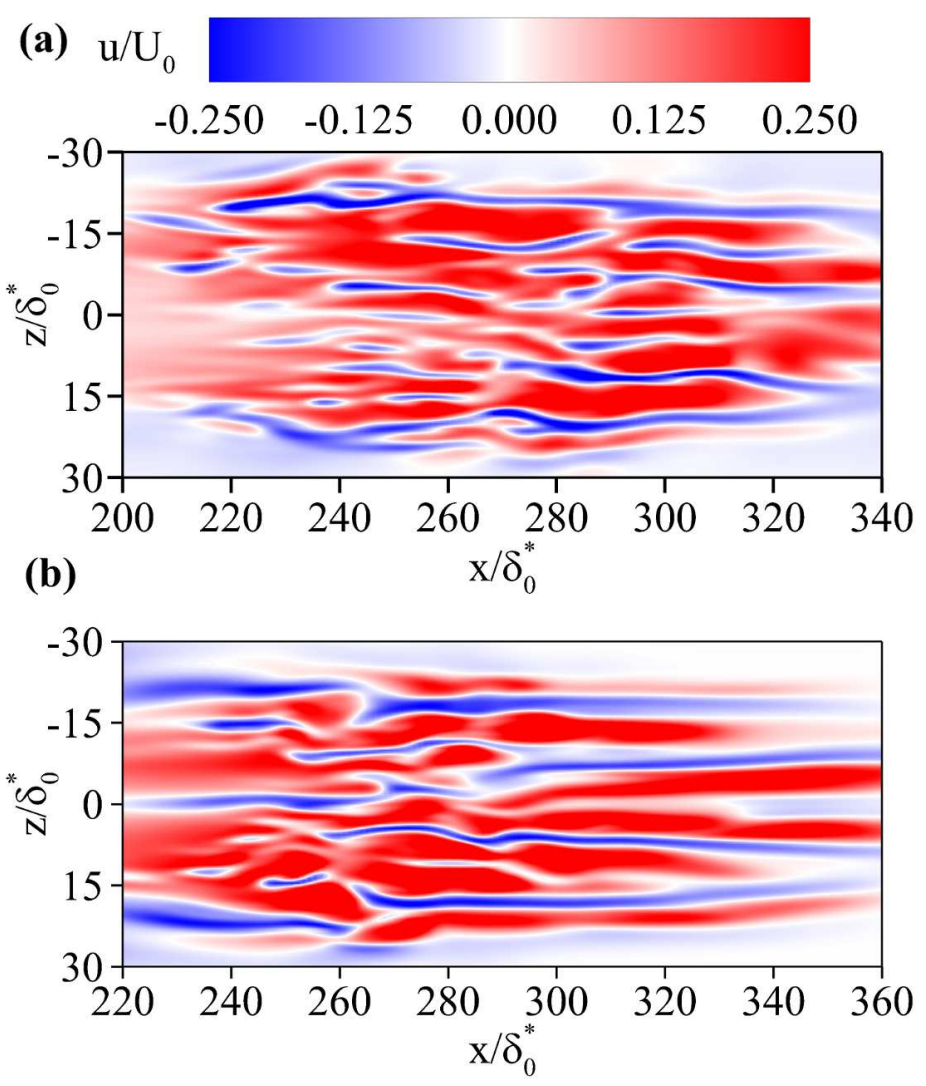

Figure 4.12: xz plane at $y / \delta_{0}^{*}=1.25$ showing low-speed steak stability in (a) Case 1 at $\tau=347$ and (b) Case 2 at $\tau=442$, when the spots are of similar size

sinuous instability mode because the spanwise-oriented density gradients are largest in the regions occupied by the shear layer along the sides of the low-speed streaks.

The average spanwise spacing of the streaks in Case 1 is $100 \pm 10$ viscous wall units, which is the value observed in Case 2 with constant fluid properties. The turbulent spot in Case 1 is exposed to a strong favourable streamwise pressure gradient, which has been shown to promote increased spanwise spacing of the streaks. Yaras (2007) and Brinkerhoff and Yaras (2011a) respectively quote spanwise spacing values of 250 and 180 viscous wall units in turbulent spots growing at a similar level of freestream acceleration as the spot in the present variable-properties case. It therefore appears that large spanwise variations in fluid density promote reduction in the spanwise spacing of the streaks, offsetting the effect of the favourable streamwise pressure gradient. 


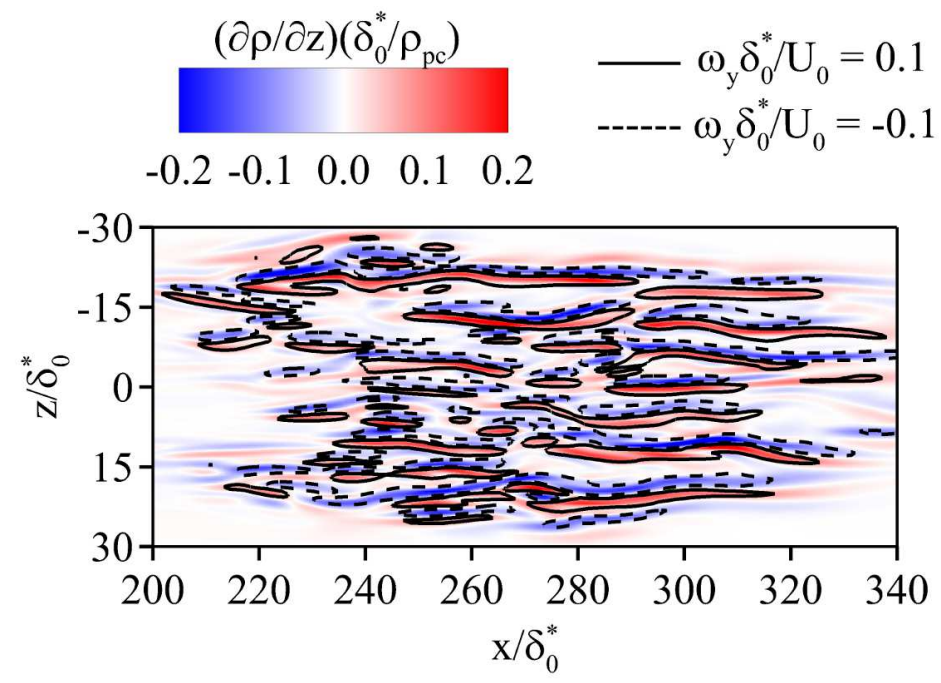

Figure 4.13: $x z$ plane at $y / \delta_{0}^{*}=1.25, \tau=347$ showing spanwise density gradients (colour contours) and wall-normal perturbation vorticity (line contours) in Case 1

This observation agrees well with the results of Azih et al. (2012), who noted reduced average streak spacing during instances of improved heat transfer in a fully-turbulent heated boundary layer. The spanwise spacing of low-speed streaks is dictated by the spacing of streamwise vortices that induce these streaks through upwash motions. Optimal spacing of these streamwise vortices allows for the maximum growth rate of hairpin vortices via local instabilities (Butler and Farrell, 1993). In a turbulent boundary layer, the optimal spacing is limited by the time scale of small-scale turbulence near the wall (Butler and Farrell, 1993). Azih et al. (2012) hypothesized that property gradients affect this time scale, thereby enhancing near-wall turbulence activity and reducing the spanwise spacing of low-speed streaks. The results of the present study suggest that this effect is primarily due to the development of instability modes driven by the density gradients in the shear layer along low-speed streaks, complementing the effects of the K-H instability mechanism.

Reduced streamwise and spanwise spacings between hairpin vortices in the spot with variable properties promote more frequent mutual interactions between these vortical structures. This involves both merging into larger structures and break-up 

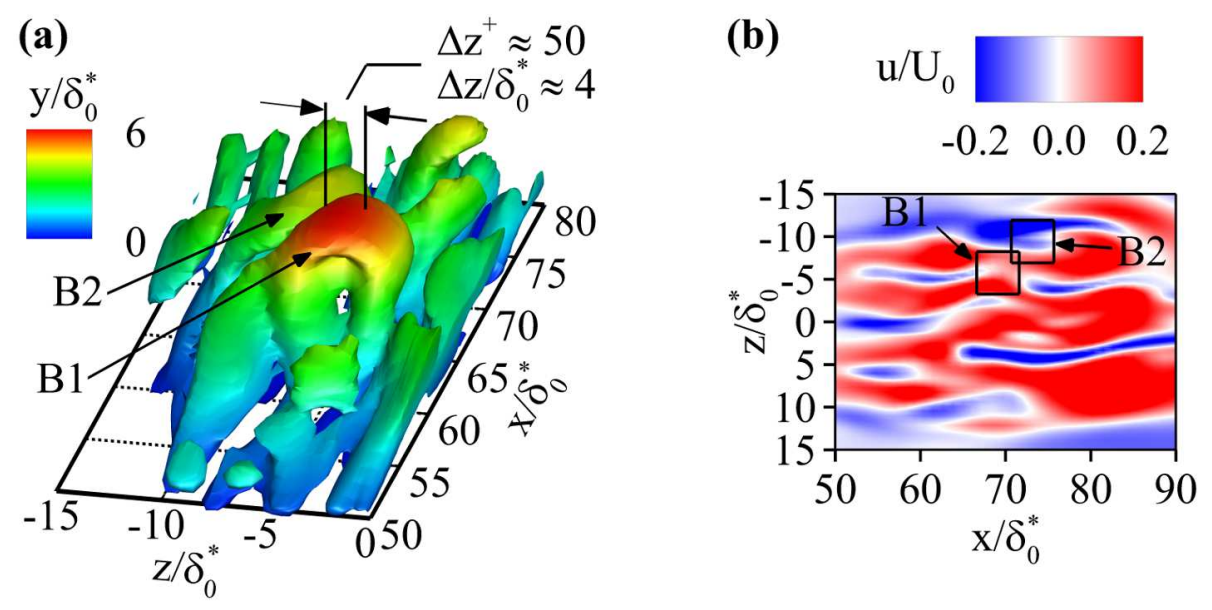

Figure 4.14: Hairpin vortices B1 and B2 immediately before they merge into hairpin vortex B1-B2 in the variable-properties case at $\tau=118$ shown with (a) iso-surfaces of $Q=2 \times 10^{-4}$ and (b) the streamwise component of perturbation velocity in the xz plane at $y / \delta_{0}^{*}=1.25$

into smaller structures. In the present study, mutual interactions are only observed between individual hairpin vortices rather than between entire wave packets, as the range of generations of wave packets of differing sizes and convection speeds is narrow due to the relatively low Reynolds number. In similar flows at higher Reynolds numbers, it is expected that, in the same way as observed amongst individual hairpin vortices, mutual interactions between generations of wave packets will be more frequent as well. The increased frequency of mutual interactions between hairpin vortices in the spot with variable properties is illustrated by following the B1-B2 hairpin vortex as it interacts with surrounding vortical structures.

The B1-B2 hairpin vortex, which was observed in Fig. 4.6, forms through the spanwise merging of the B1 and B2 hairpin vortices. As illustrated in Fig. 4.14, this merging is promoted by local reduction in the spanwise spacing of low-speed streaks, which is driven by enhanced sinuous instability of the streaks as discussed earlier. Fig. 4.15 shows that two tertiary hairpin vortices, labelled E1 and E2, form about 110 viscous wall units downstream of the B1-B2 secondary hairpin vortex and upstream of the primary hairpin vortex A. Because of the close streamwise spacing 

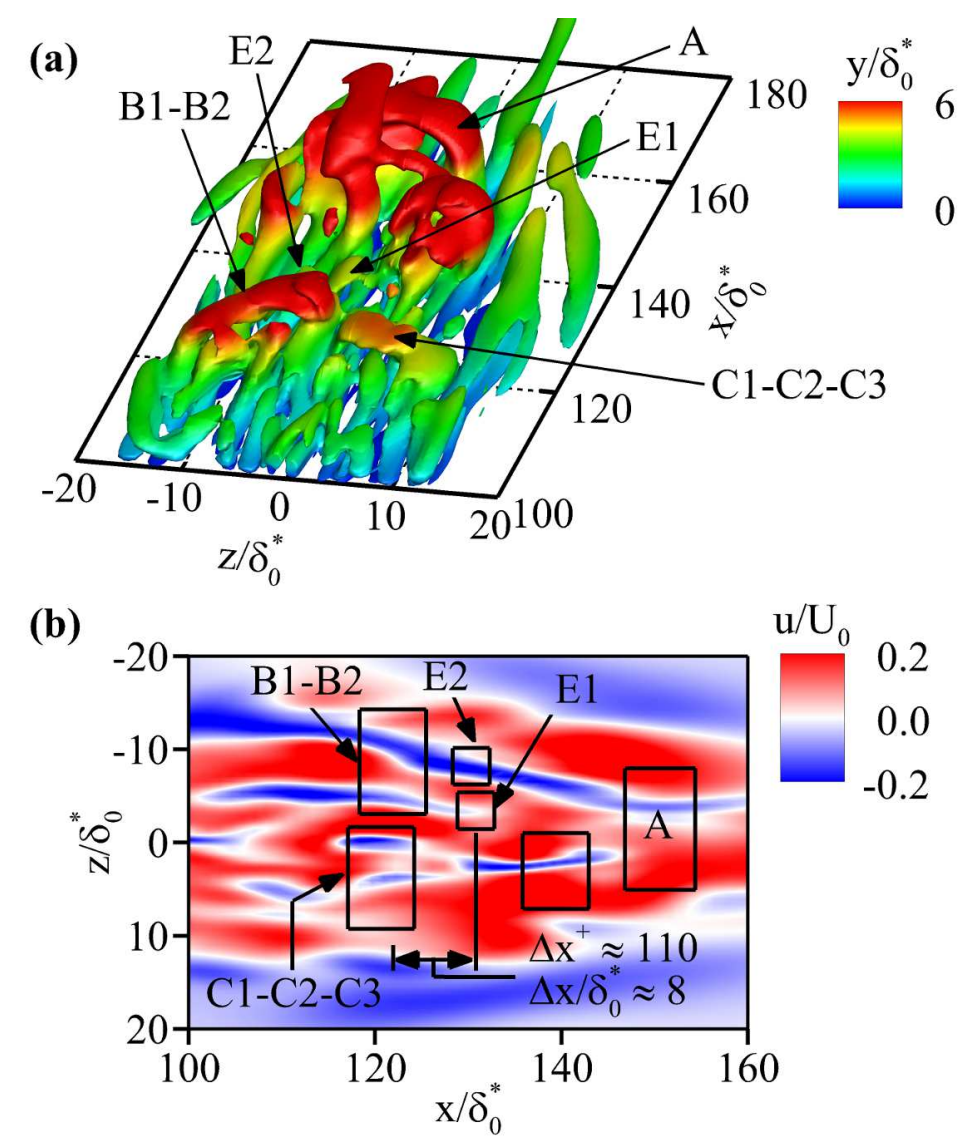

Figure 4.15: Development of hairpin vortices E1 and E2 downstream of B1B2 in the variable-properties case at $\tau=175$ presented through (a) iso-surfaces of $Q=2 \times 10^{-4}$ and (b) the streamwise component of perturbation velocity in the $\mathrm{xz}$ plane at $y / \delta_{0}^{*}=1.5$

between E1, E2, and B1-B2, the downwash induced by the head of B1-B2 initially prevents E1 and E2 from growing in the wall-normal direction. As a result, the fastertravelling B1-B2 hairpin vortex overtakes the E1 and E2 hairpin vortices. As B1-B2 passes over E2, the strong upwash motion between its legs causes E2 to extend in the wall-normal direction until its head merges with the head of B1-B2, as shown in Fig. 4.16a. Nearly simultaneously, the head and $-\mathrm{z}$ leg of E1 merge with the $+\mathrm{z}$ side of B1-B2. The $+\mathrm{z}$ leg of E1 breaks up C1-C2-C3 into two smaller structures labelled C4 and C5 as illustrated in Fig. 4.16c, which join with the newly-formed B1-B2-E1-E2 structure. This larger hairpin vortex structure now spans over half the 

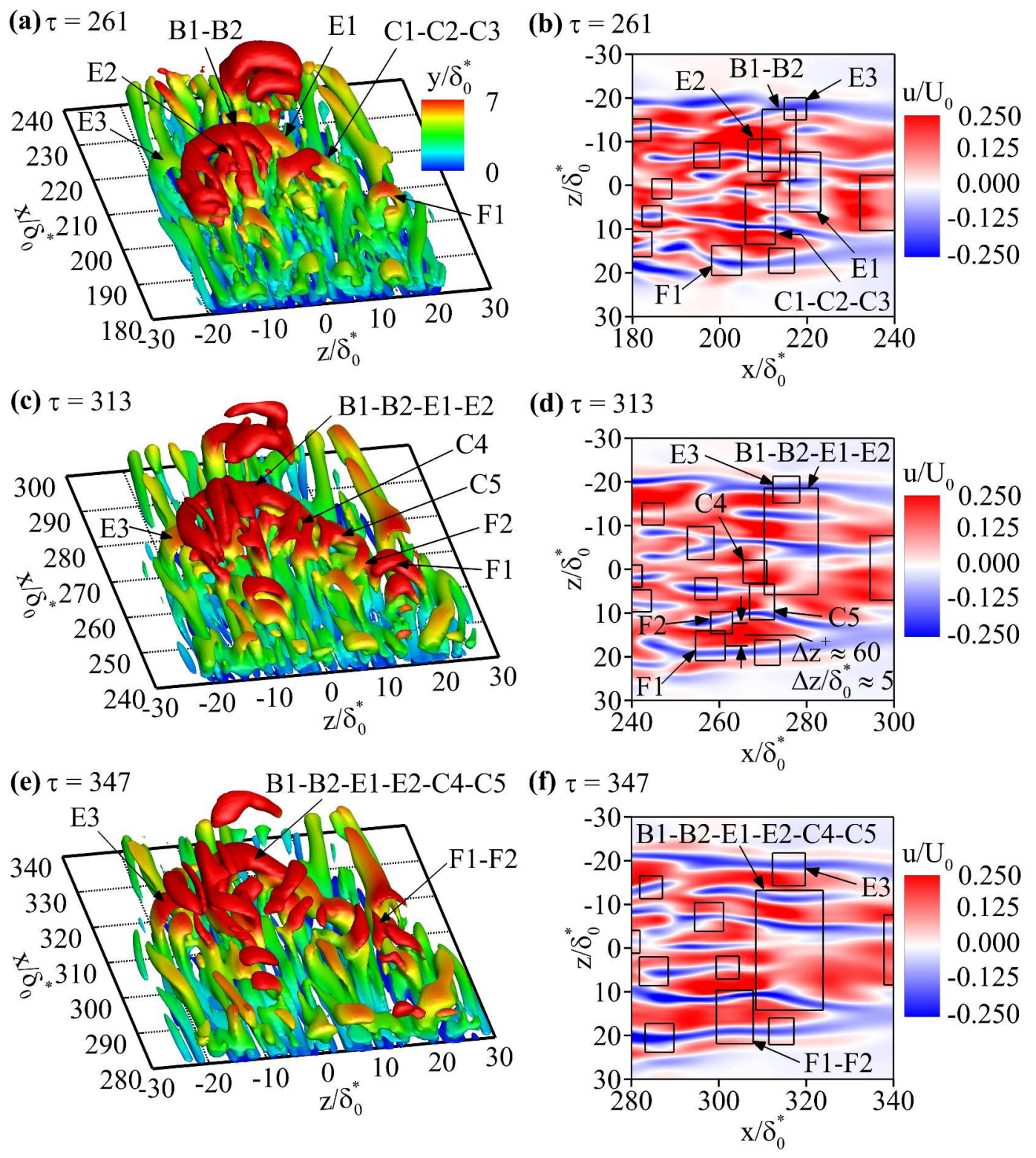

Figure 4.16: Interactions between hairpin vortices in the turbulent spot of Case 1, presented using iso-surfaces of $Q=2 \times 10^{-4}$ and the streamwise component of perturbation velocity in the $\mathrm{xz}$ plane at $y / \delta_{0}^{*}=1.5$ 
width of the spot. Fig. 4.16c also shows the F1 and F2 hairpin vortices developing on the far $+z$ side of the spot. The spanwise spacing between these hairpin vortices is only about 60 viscous wall units due to spanwise oscillations in the streaks over which they have formed, which causes them to merge together. The resulting hairpin vortex, F1-F2, is then broken up by the $+\mathrm{z}$ leg of the B1-B2-E1-E2-C4-C5 structure at $\tau=347$.

Returning to $\tau=261$, a third tertiary hairpin vortex forms a short distance downstream of B1-B2, labelled E3. As with E1 and E2, E3 is unable to grow in the wall-normal direction because of the downwash motions induced by the head of the B1-B2 hairpin vortex. As B1-B2 overtakes the slower-moving E3 hairpin vortex, the upwash motion induced by B1-B2 causes E3 to extend away from the wall as shown in Fig. 4.16c. Consequently, the $-\mathrm{z}$ leg of the B1-B2-E1-E2-C4-C5 structure breaks off and joins with E3 to form a separate large hairpin vortex at $\tau=347$.

In the turbulent spot with constant thermophysical properties, similar tertiary hairpin vortices form between secondary hairpin vortices and the primary hairpin vortex at the leading edge of the spot. These tertiary hairpin vortices are spaced about 225 to 350 viscous wall units apart in the streamwise direction as shown in Fig. 4.17, and this streamwise spacing does not change substantially from $\tau=398$ to $\tau=497$. As a result, these tertiary hairpin vortices are able to grow in the wall-normal direction without being inhibited by the downwash motions induced by the heads of the secondary hairpin vortices. Contrary to the spot with variable properties, the tertiary hairpin vortices grow to a wall-normal height similar to that of the secondary hairpin vortices and convect downstream at a similar speed as the secondary hairpin vortices such that they are not overtaken by them. The low-speed streaks and the hairpin vortices which form over them remain fairly well-aligned, and the streaks only exhibit instability closer to the trailing edge of the spot. Hence the hairpin vortices are not observed to mutually-interact with neighbouring hairpin vortices which have 


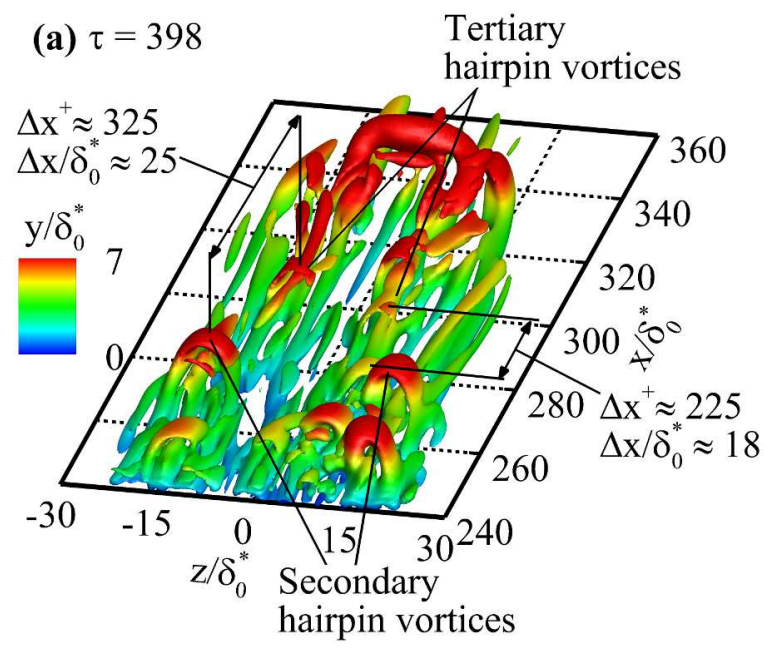

(b) $\tau=398$

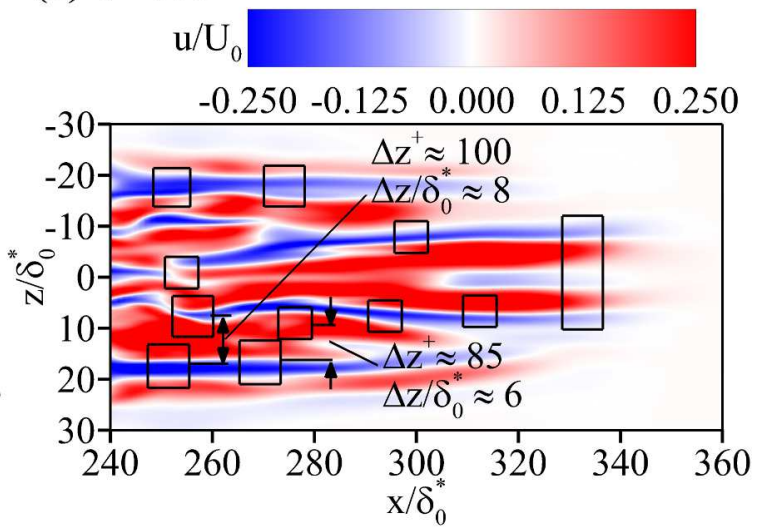

(c) $\tau=497$

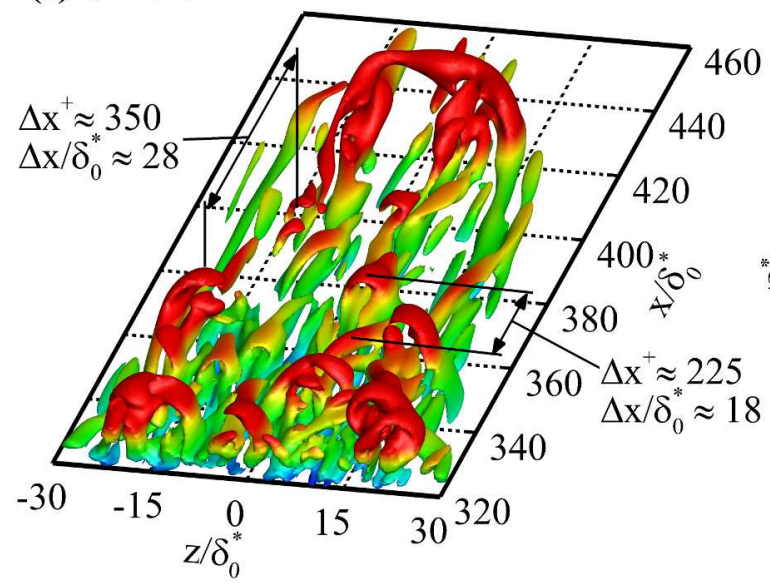

(d) $\tau=497$

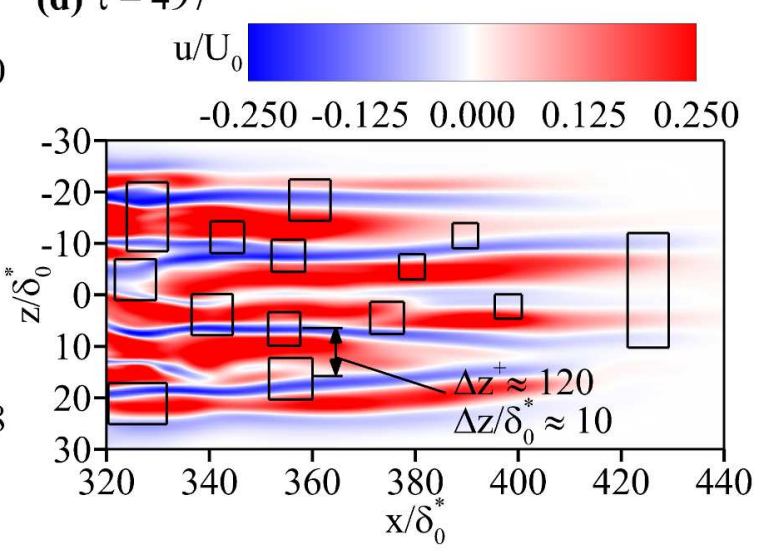

Figure 4.17: Streamwise spacing between hairpin vortices in the case with constant properties shown using iso-surfaces of $Q=2 \times 10^{-4}$ and the streamwise component of perturbation velocity in the xz plane at $y / \delta_{0}^{*}=1.5$

formed over adjacent low-speed streaks.

Fig. 4.18 schematically summarizes the observations in the turbulent spot discussed in this section. The physical mechanisms by which the density gradients promote the K-H instability mechanism resulting in the trends observed in Fig. 4.18, and the relative contributions of the density and viscosity gradients to these trends, are examined next. 

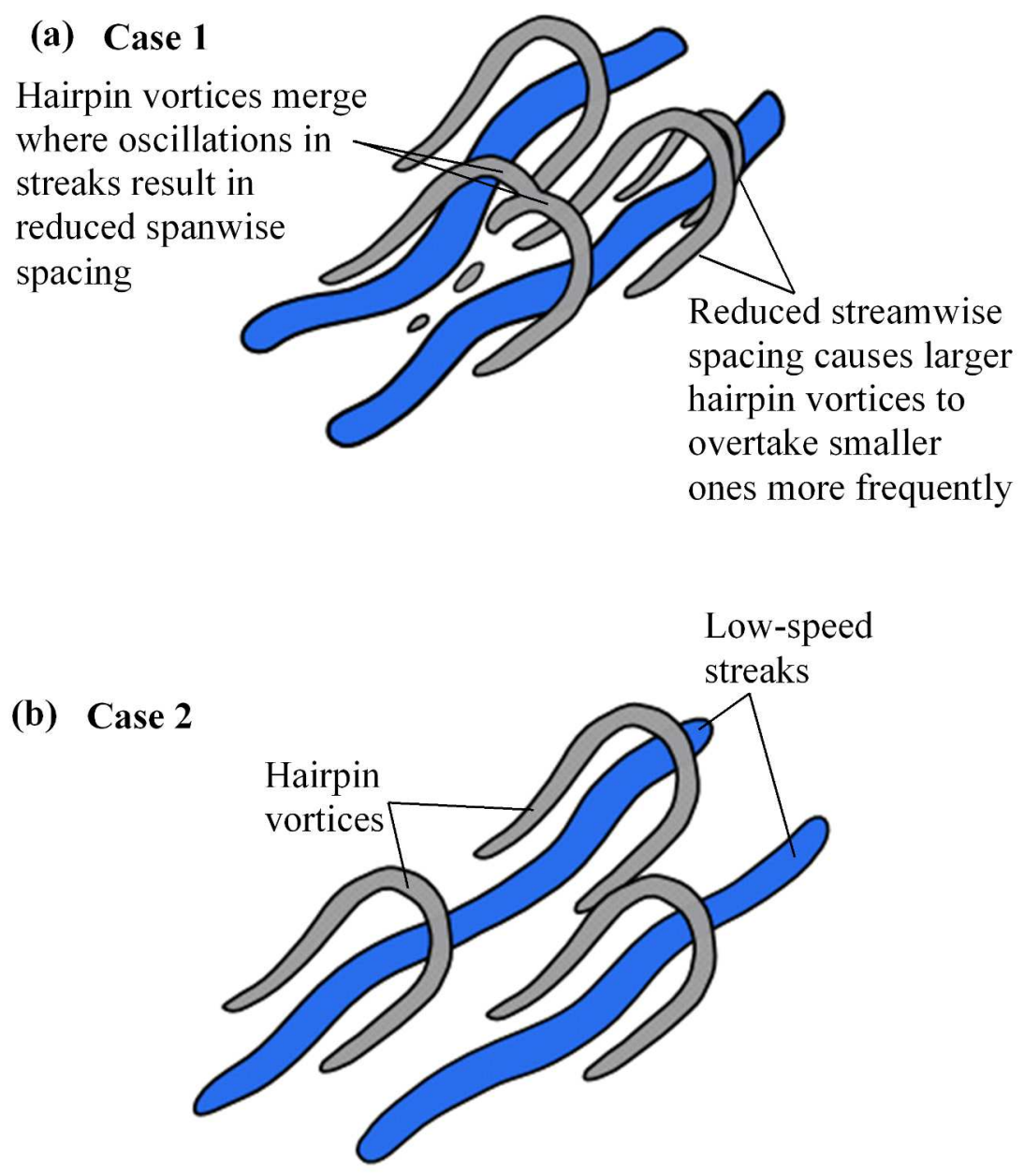

Figure 4.18: Schematic comparing the topology of hairpin vortices in the spot with variable properties (Case 1) and constant properties (Case 2)

\subsection{Effects of cross-stream density and viscosity gradients on the K-H instability mechanism}

Two-dimensional free shear layer instability simulations presented in this section provide an idealized environment for identifying the physical mechanisms by which density and/or viscosity gradients promote the $\mathrm{K}-\mathrm{H}$ instability mechanism in the shear layer along low-speed streaks.

The boundary layer at the trailing edge of the splitter plate provides the initial 


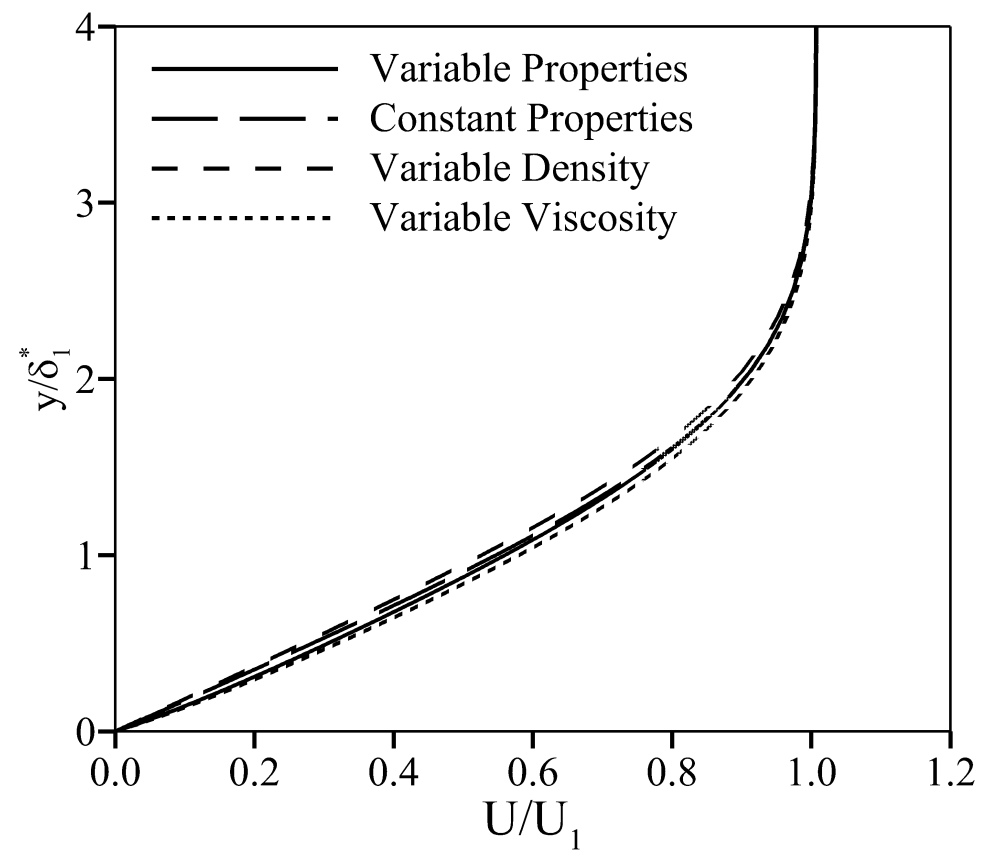

Figure 4.19: Wall-normal profile of streamwise velocity in the high-speed stream at the splitter-plate trailing edge

conditions for the development of the free shear layer downstream of the trailing edge. The wall-normal profile of streamwise velocity in the high-speed stream at the trailing edge of the splitter plate is shown in Fig. 4.19. In the figure, $U_{1}$ is the local freestream velocity and $\delta_{1}^{*}$ is the local displacement thickness. The lower viscosity near the surface in the Variable Viscosity case creates a slightly fuller velocity profile compared to the Constant Properties case. A velocity deficit exists in the Variable Density case; the mechanism responsible for this is illustrated schematically in Fig. 4.20. As the velocity profile develops in response to the no-slip condition, the flow decelerates in the streamwise direction, indicated by the $\vec{a}$ vector. The deceleration vector is perpendicular to the positive wall-normal density gradient, denoted by the $\nabla \rho$ vector, which results in the production of negative vorticity by the baroclinic vorticity generation mechanism. The additional vorticity induces a streamwise component of velocity which opposes the flow near the surface, thereby creating a velocity deficit. The velocity profile for the Variable Properties case is noted to be slightly fuller than 


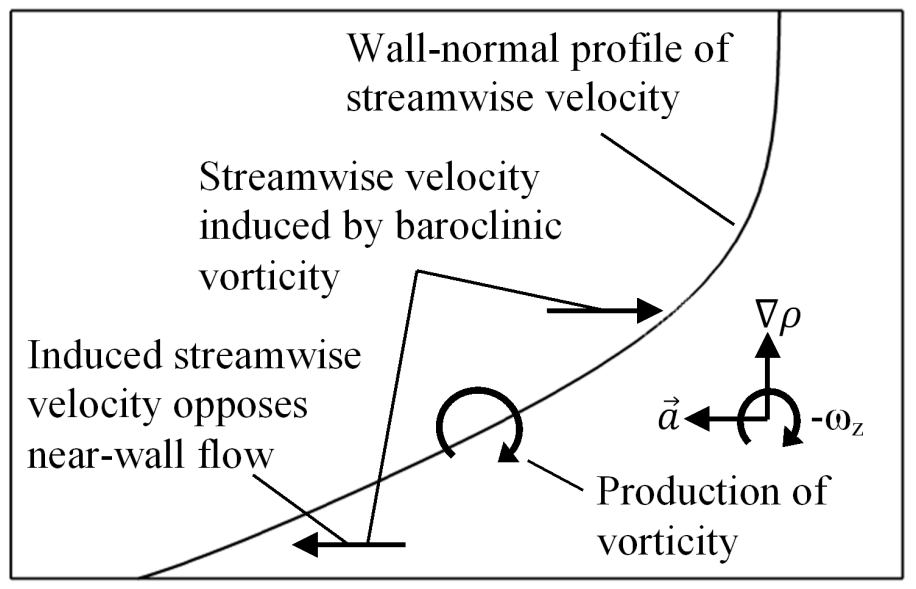

Figure 4.20: Illustration of the effect of a wall-normal density gradient on the wallnormal streamwise velocity profile (not to scale)

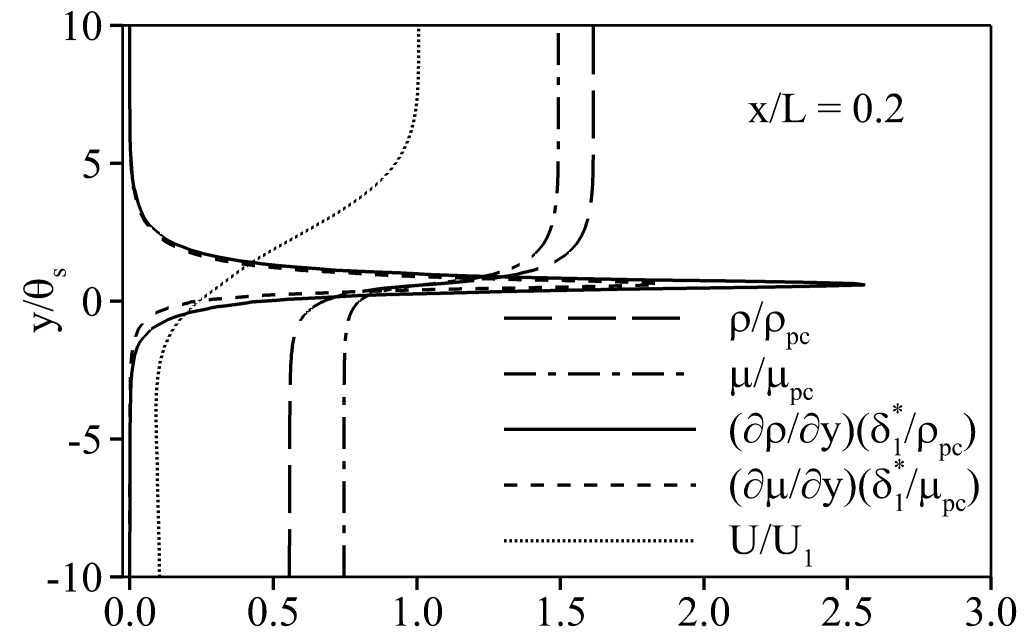

Figure 4.21: Cross-stream distributions of density and viscosity and their crossstream gradients

the profile for the Constant Properties case. This suggests that the effect of reduced viscosity on the velocity profile more than offsets the effect of reduced density.

The undisturbed cross-stream distributions of density and viscosity and their cross-stream gradients $20 \%$ splitter-plate length downstream of the trailing edge in the Variable Properties case are presented in Fig. 4.21. $\theta_{s}$ is the local momentum thickness of the free shear layer. When the property gradients are normalized using 

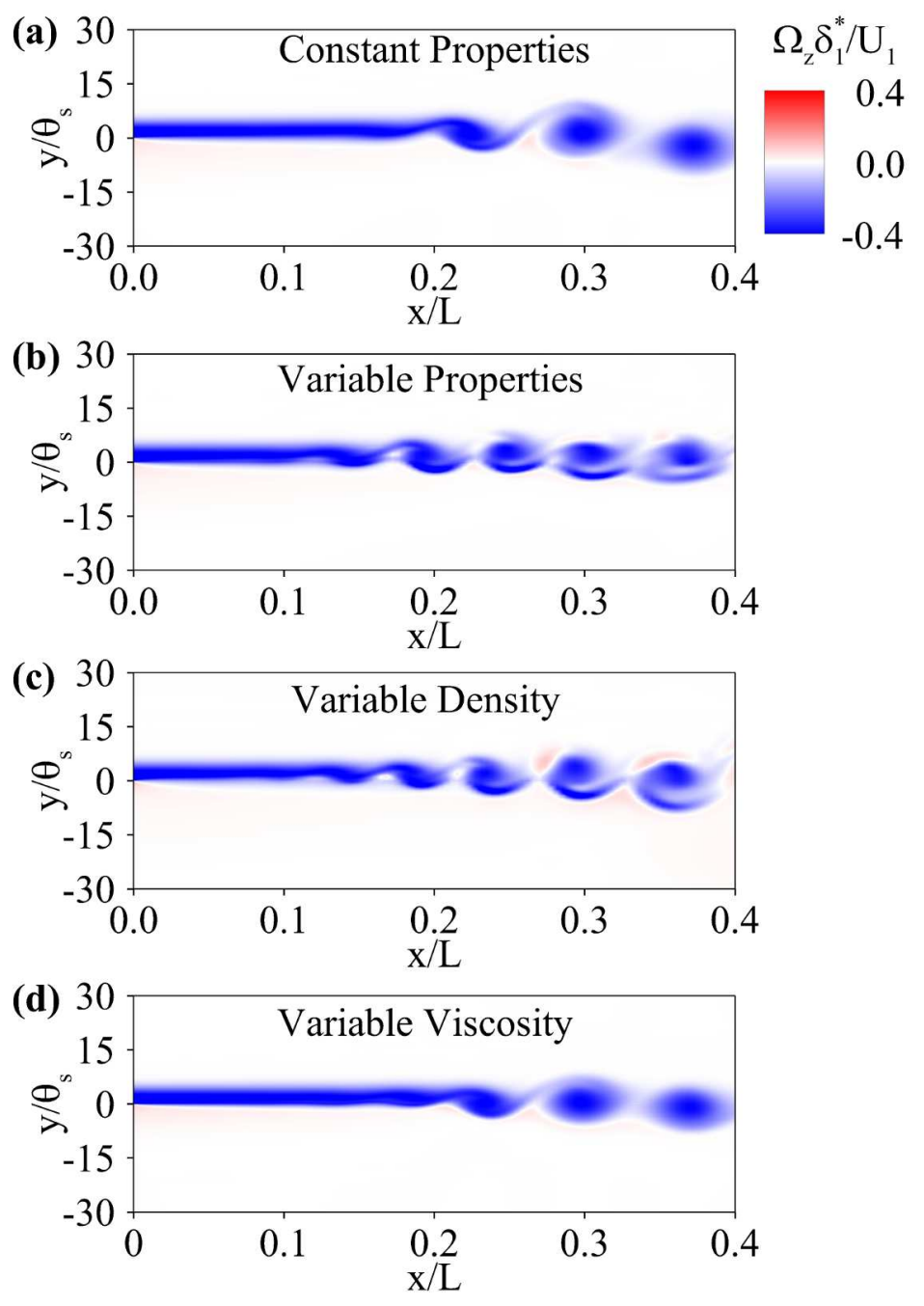

Figure 4.22: Development of the free shear layer visualized through the spanwise component of vorticity, $\Omega_{z} \cdot x / L=0$ : splitter-plate trailing edge.

$\delta_{1}^{*}$ to facilitate comparison with those in the simulated turbulent spot, they are an order of magnitude larger than those in the spot. These choices of property gradients ensure that the mechanisms by which they affect the roll-up of the free shear layer are clearly identifiable.

Instability of the free shear layer through the $\mathrm{K}-\mathrm{H}$ mechanism leading to the roll-up into $\mathrm{K}-\mathrm{H}$ vortices is observed in Fig. 4.22. In the Variable Properties and Variable Density cases, the streamwise spacing of the $\mathrm{K}-\mathrm{H}$ vortices is reduced and 
Table 4.1: Strouhal number of K-H vortex shedding

\begin{tabular}{cc} 
Case & Strouhal number \\
\hline Constant Properties & 0.032 \\
Variable Properties & 0.049 \\
Variable Density & 0.049 \\
Variable Viscosity & 0.032 \\
\hline
\end{tabular}

the completion of the roll-up of the free shear layer occurs closer to the trailing edge compared to the Variable Viscosity and Constant Properties cases. The Strouhal number of the formation of $\mathrm{K}-\mathrm{H}$ vortices, $\mathrm{St}=f \theta_{s} / U_{K H}$, is listed in Table 4.1. $f$ is the frequency; $\theta_{s}$ is the momentum thickness at $x / L=0.13$ in the Variable Properties and Variable Density cases and at $x / L=0.23$ in the Constant Properties and Variable Viscosity cases; and $U_{K H}$ is the convection speed of the K-H vortices. The frequency is calculated over 10 vortex shedding cycles using the cross-stream velocity component at $y / \theta_{s}=2$, which is the cross-stream centre of the free shear layer in a time-averaged sense. The evaluation was repeated over five different time intervals, yielding an uncertainty of \pm 0.001 for the tabulated values of Strouhal numbers.

The shedding frequency in the Constant Properties case agrees well with the value of 0.032 quoted in published literature for free shear layers with uniform fluid properties (Ho and Huerre, 1984). The consistency of the Strouhal number value for the Variable Viscosity and Constant Properties cases suggests that the viscosity gradients considered in these tests do not have a visible effect on the stability characteristics of the free shear layer. It is worth noting that this agreement is achieved despite the slightly fuller velocity profile for the Variable Viscosity case as presented in Fig. 4.19. The $\sim 55 \%$ increase in the shedding frequency observed for the Variable Density and Variable Properties cases is therefore the result of the cross-stream density gradients.

Fig. 4.23 presents the streamwise growth of streamwise perturbation velocity fluctuations, evaluated over 10 vortex shedding cycles. The uncertainty shown by error 


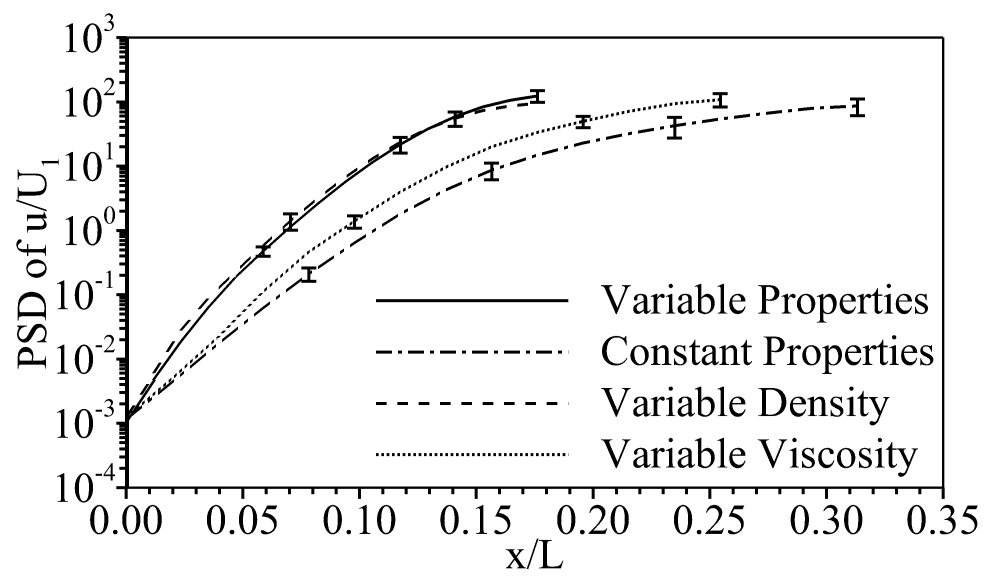

Figure 4.23: Streamwise variation of the power spectral density of streamwise velocity fluctuations at the shedding frequency of the K-H vortices evaluated at $y / \theta_{s}=2$

bars is established by performing the calculation over five different time intervals. The trends suggest that roll-up into $\mathrm{K}-\mathrm{H}$ vortices is accelerated by cross-stream density gradients, which is consistent with the reduced streamwise spacing of the $\mathrm{K}-\mathrm{H}$ vortices observed for the Variable Density and Variable Properties cases in Fig. 4.22 and Table 4.1. The roll-up of the free shear layer into a K-H vortex must be initiated by a perturbation. The primary source of this perturbation is the induced cross-stream velocity from the accumulated vorticity in the K-H vortex developing one wavelength downstream. The faster each K-H vortex develops, the sooner it can initiate the development of the next K-H vortex by a cross-stream velocity perturbation induced upstream, thus affecting the streamwise spacing of the $\mathrm{K}-\mathrm{H}$ vortices in the wake of the splitter plate.

Several studies have examined the influence of baroclinic vorticity generation on the vorticity distribution within K-H vortices in free shear layers of nonuniform density, but they do not consider the roll-up process (Soteriou and Ghoniem, 1995; Reinaud et al., 2000, 1999). Fig. 4.24 illustrates how baroclinic vorticity generation likely accelerates the perturbation growth rate leading to the formation of a $\mathrm{K}-\mathrm{H}$ vortex through the shear-layer roll-up process. In the figure, $\vec{a}$ is the acceleration of fluid 

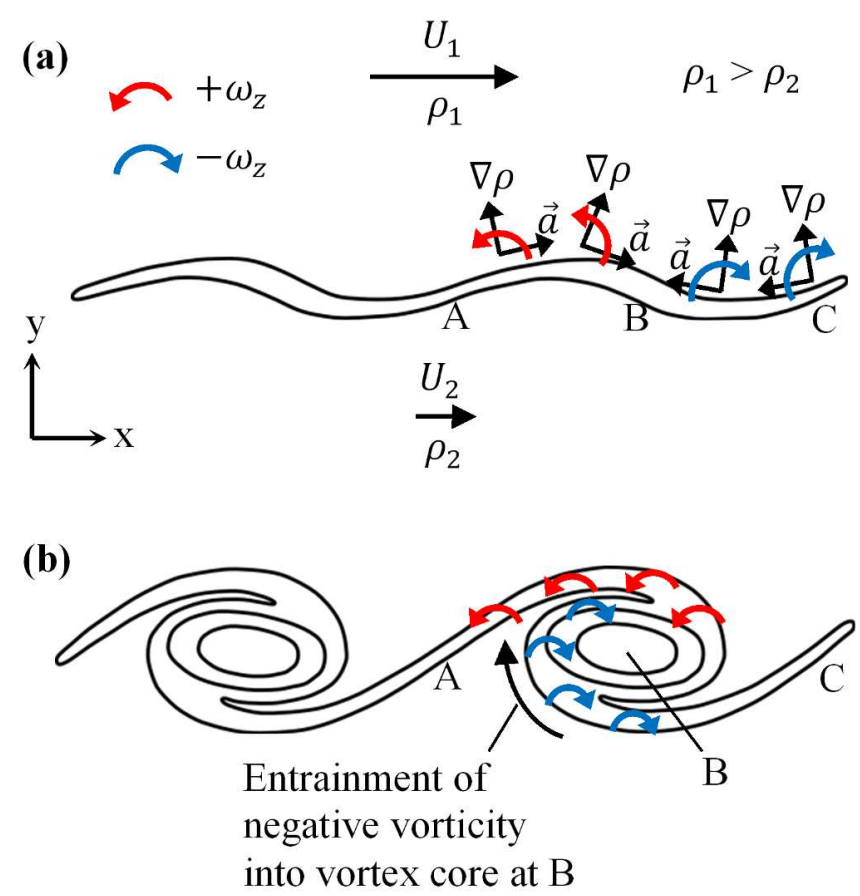

Figure 4.24: Illustration of the baroclinic vorticity generation mechanism by which cross-stream density gradients affect the roll-up of a free shear layer into K-H vortices

particles in the direction of local fluid motion and $\omega_{z}$ represents the spanwise vorticity generated by the baroclinic mechanism. The streamwise grouping of spanwise vorticity in an inviscid-unstable free shear layer is the result of local streamwise accelerations which carry spanwise vorticity of negative sign away from points A and C and toward point B (Drazin and Reid, 2004). When the cross-stream density gradient is in the same direction as the cross-stream velocity gradient, the local acceleration of fluid particles from A to B generates positive vorticity by the baroclinic vorticity generation mechanism, whereas the local acceleration of fluid particles from $\mathrm{C}$ to $\mathrm{B}$ generates negative vorticity. While the positive vorticity generated between A and B partially cancels the background negative vorticity in the free shear layer, the negative vorticity generated between $\mathrm{C}$ and $\mathrm{B}$, which is additional to the background negative vorticity, is convected into the centre of the vortex core by the rotational motion of the free shear layer as it folds over during the roll-up process. This accelerates the 
accumulation of negative vorticity at point B, which causes the free shear layer to roll up into $\mathrm{K}-\mathrm{H}$ vortices at a faster rate.

The study of baroclinic vorticity generation in free shear layers in the published literature has focused primarily on cases involving two different working fluids with a density-profile thickness that is comparable to the velocity-profile thickness of the free shear layer (Davey and Roshko, 1972; Brown and Roshko, 1974; Soteriou and Ghoniem, 1995; Reinaud et al., 2000). In contrast, the density-profile thickness in the present study is about $45 \%$ of the velocity-profile thickness immediately downstream of the splitter-plate trailing edge. The reduced density-profile thickness results in a larger magnitude of the cross-stream density gradient. This difference is likely the explanation for the effects of baroclinic vorticity generation on the shear-layer roll-up in the present study to be notably stronger than those observed in the published literature.

A second potential mechanism that may contribute to the modification of the rollup of the free shear-layer into K-H vortices is the Holmboe instability (John, 2012). In a free shear layer at a very similar thermodynamic state as in the present study, John (2012) observed the formation of a pair of counter-rotating spanwise vortices by the Holmboe instability mechanism between $\mathrm{K}-\mathrm{H}$ vortices. These Holmboe vortices form due to the centripetal acceleration experienced by fluid particles as a result of the curvature in the free shear layer created by the $\mathrm{K}-\mathrm{H}$ instability mechanism. A necessary condition for the formation of Holmboe vortices is for the Richardson number to have a value between about 0.025 and 0.5 (Lawrence et al., 1991; John, 2012). In the present study, the Richardson number based on the local centripetal accelerations experienced by fluid particles reaches a maximum value of about 0.01 , which is an order of magnitude lower than the value of 0.16 realized by John (2012). This difference is caused by the significantly lower streamwise velocities of the present study compared to those in John's study. Because of the very low values of the 
Richardson number, Holmboe vortices do not develop in the present cases.

The physics explored in the roll-up of a free shear layer in the present section provide guidance in the analysis of the shear-layer development along low-speed streaks in the turbulent spots described in sections 4.2 and 4.3. The frequency at which the shear layers enveloping low-speed streaks roll up into hairpin vortices is computed using the convection velocity and streamwise spacing of hairpin vortices as they form within a wave packet. The frequency is normalized using the local momentum thickness of the shear layer surrounding the low-speed streak and the convective velocity of the rollers developing into hairpin vortices. In the spot with constant properties, the average Strouhal number is evaluated to be $0.013 \pm 0.001$ using five different wave packets. The average Strouhal number in the spot with variable properties is $0.019 \pm 0.001$ based on eight wave packets, which is $\sim 45 \%$ greater than in the case with constant properties. The Strouhal numbers in the variable-property and constant-property turbulent spots are both lower than the variable-property and constant-property free shear layers. In the spots, the cross-stream velocity component in the shear layer above the streaks is suppressed by the nearby no-slip surface (Brinkerhoff et al., 2011; John, 2012). In the shear layer on the sides of the streaks, similar suppression occurs due to the cross-stream velocity induced by the roll-up of the shear layer on the opposite side of the streak. This suppression has a stabilizing effect, leading to reduced values of Strouhal number in the development of the shear layer enveloping low-speed streaks (Brinkerhoff et al., 2011; John, 2012). The percentage increase in the Strouhal number in the free shear layer simulations due to the density gradients is only about $10 \%$ higher than the percentage increase observed in the turbulent spot simulations, even though the density gradients in the free shear layer simulations are an order of magnitude larger. High values of density gradients in the free shear layer simulations are confined to within less than $5 \%$ of the shear layer thickness, which provides a plausible explanation for the noted trend. The local 
Richardson number in the regions where the shear layer enveloping low-speed streaks rolls up into hairpin vortices does not exceed 0.01, such that Holmboe vortices do not form in the turbulent spot with variable properties.

\subsection{Turbulent spot spreading rates}

The degree to which spatial variations in density promote the generation of hairpin vortices can be estimated by comparing the spreading rate of the spot with variable and constant properties. Brinkerhoff and Yaras (2014) demonstrated that the spreading of a turbulent spot is primarily driven by the generation of hairpin vortices along the edges of the spot, and that the development of such hairpin vortices follows the same fundamental physical processes as that within the interior of the spot, as reviewed in section 4.2. As was demonstrated in section 4.1, wall-normal variations in density and viscosity do not significantly affect the stability of the undisturbed laminar boundary layer, which is dominated by the strong favourable streamwise pressure gradient. This implies that the spreading rate of the turbulent spot in the variableproperties case is dominantly affected by the favourable streamwise pressure gradient and the generation of new wave packets of hairpin vortices along the sides, leading edge, and trailing edge of the spot.

To analyze the growth rate of the turbulent spot, a precise method to identify the boundary between the laminar and turbulent flow regions is required. The leading edge, trailing edge, and the two side edges of a turbulent spot are typically identified as the farthest locations where a flow variable that is nominally zero in undisturbed laminar flow regions exceeds a threshold value. In the published literature, the streamwise component of the perturbation velocity, $u / U_{0}$, is used most frequently to locate the perimeter of a turbulent spot (Katz et al., 1990; Wygnanski et al., 1976; Coles and Barker, 1975; Glezer et al., 1989). In the present study, this approach is found to 

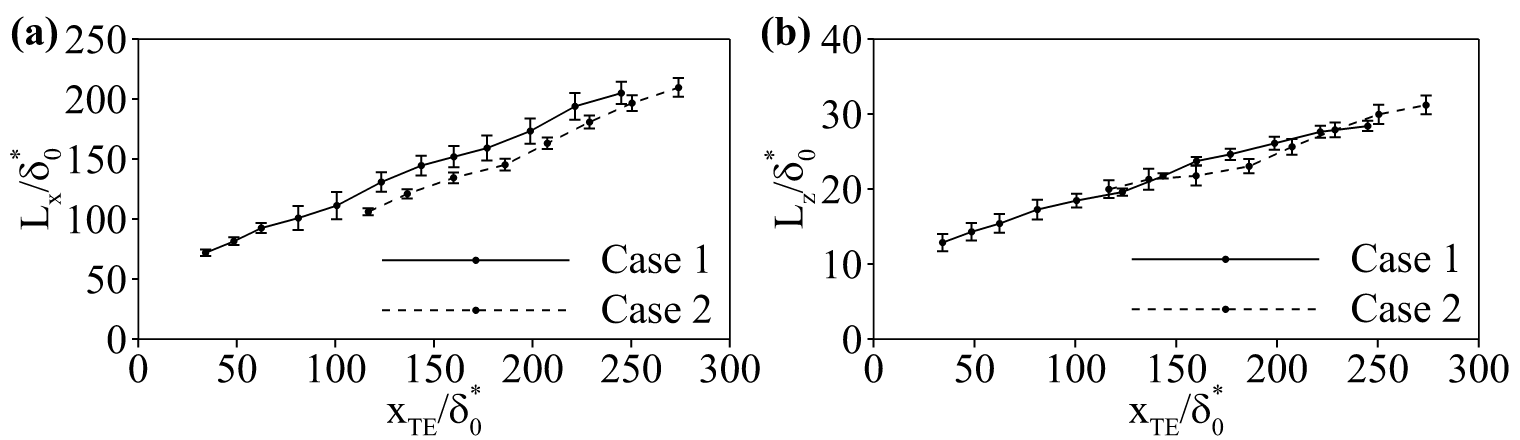

Figure 4.25: Streamwise variation of (a) turbulent spot length and (b) turbulent spot half-width. $x_{T E}$ : streamwise location of the spot trailing edge.

be more effective than the streamwise vorticity that was utilized by Brinkerhoff and Yaras (2014) for the constant-properties case, particularly during the early phases of development of the spot. Four threshold values of $0.10,0.15,0.20$, and 0.25 for $u / U_{0}$ are used at each spot edge; the four locations corresponding to the four threshold values are averaged to yield one location at each edge of the spot. Subsequently, the edge locations on the two sides are averaged to provide an average half-width of the spot, $L_{z} / \delta_{0}^{*}$, and the trailing edge location is subtracted from the leading edge location to yield the streamwise length of the spot, $L_{x} / \delta_{0}^{*}$. The sensitivity of this method to the number of threshold values used was determined by repeating the calculations with threshold values of $u / U_{0}=0.100,0.125,0.150,0.175,0.200,0.225$, and 0.250 . Sensitivity of the method to the range of threshold values was established by repeating the calculations for $u / U_{0}$ ranges of $0.025-0.325,0.05-0.20$, and $0.15-0.30$.

The streamwise variation of the length and half-width of the turbulent spot calculated with the described approach is presented in Fig. 4.25, including error bars reflecting the results of the noted sensitivity analysis. Within the uncertainty bounds, the growth rates are about the same for the spot with variable and constant properties. The spot with variable properties is exposed to a stabilizing favourable streamwise pressure gradient. Similar growth rates between the spot with variable and constant properties therefore imply that the increased rate and frequency of hairpin 
Table 4.2: Turbulent spot spreading rates with and without a favourable streamwise pressure gradient

\begin{tabular}{ccccc}
\hline & $\begin{array}{c}\text { Falkner-Skan } \\
\text { parameter }\end{array}$ & $d L_{z} / d x$ & $d L_{x} / d x$ & $\mathrm{Re}_{\delta_{0}^{*}}$ \\
\hline Sankaran et al. (1986) & 0.095 & 0.128 & 0.32 & $\sim 450$ \\
& 0 & 0.158 & 0.38 & \\
\hline Wygnanski (1981) & 0.12 & 0.0875 & 0.29 & $\sim 500$ \\
\hline Katz et al. (1990) & 0 & 0.176 & 0.45 & \\
\hline Case 1 & 1.0 & 0.105 & 0.35 & $\sim 1000$ \\
Case 2 & 0 & 0.195 & $\sim 0.70$ & \\
\hline
\end{tabular}

vortex formation due to spatial gradients in density offsets the stabilizing effect of the favourable streamwise pressure gradient. Published results on the isolated effect of favourable streamwise pressure gradient on the spot spreading rate are presented in Table 4.2. The Falkner-Skan parameter is the value corresponding to the streamwise velocity profile of the Falkner-Skan solution which matches the laminar boundary layer streamwise velocity profile in each study. The data in the table demonstrates that for favourable streamwise pressure gradients with magnitudes similar to that of the present study, in the absence of spatial gradients in density, the spot spreading rate is reduced by as much as $50 \%$ compared to the case of zero streamwise pressure gradient. Furthermore, Brinkerhoff and Yaras (2011a) and Yaras (2007) observed that turbulent spots growing in flow fields with uniform thermophysical properties undergoing a similar level of freestream acceleration at a similar Reynolds number as the spot with variable properties in the present study begin to re-laminarize. 


\section{Chapter 5}

\section{Conclusions and Recommendations}

\subsection{Conclusions}

The results of the present study show that in a boundary layer with large wall-normal thermophysical property gradients, large spatial gradients in density promote the formation of hairpin vortices leading to a higher concentration of coherent structures of turbulence, which results in improved wall-normal mixing, and hence improved convection heat transfer. The density gradients promote the $\mathrm{K}-\mathrm{H}$ instability mechanism in the inviscid-unstable shear layer enveloping low-speed streaks through the baroclinic vorticity generation mechanism. This accelerates the streamwise grouping of vorticity in this shear layer, which causes it to roll up into hairpin vortices at a faster rate and at a higher frequency. The increased roll-up frequency of hairpin vortices leads to a reduction in streamwise spacing between hairpin vortices in wave packets. The density gradients also promote the sinuous instability mode in low-speed streaks, such that oscillations in the streaks in the streamwise-spanwise plane bring about locally reduced spanwise spacing between hairpin vortices which form over adjacent streaks. Because of the reduction in streamwise and spanwise spacing between them, hairpin vortices are observed to mutually interact with each other more frequently by merging together into larger structures and by breaking apart into smaller structures. 


\subsection{Recommendations for future work}

It is recommended that similar simulations be performed in future studies to identify the physical mechanisms by which large wall-normal property gradients modify the development of coherent structures of turbulence during deteriorated heat transfer. To simulate deteriorated heat transfer, the simulations of the present study would be repeated with zero streamwise pressure gradient, an inflow temperature below the pseudocritical temperature, and a significantly greater value of heat flux-to-mass flux ratio. Azih and Yaras (2013) observed deteriorated heat transfer in a heated channel flow with supercritical water in the absence of buoyancy forces with an inflow

temperature of $T=643 \mathrm{~K}$ and a heat flux-to-mass flux ratio of $q_{w}^{\prime \prime} / G=3.0 \mathrm{~kJ} / \mathrm{kg}$. It would also be worthwhile to perform similar simulations to examine improved and deteriorated heat transfer under high freestream turbulence to determine if the physical mechanisms by which fluid-property variation affects the development of turbulence structures are as pronounced. 


\section{References}

Adrian, R. J., Meinhart, C. D., and Tomkins, C. D. (2000). Vortex organization in the outer region of the turbulent boundary layer. J. Fluid Mech., 422, 1-54.

Azih, C., Brinkerhoff, J. R., and Yaras, M. I. (2012). Direct numerical simulation of convective heat transfer in a zero-pressure-gradient boundary layer with supercritical water. J. Therm. Sci., 21, 49-59.

Azih, C., and Yaras, M. I. (2013). Direct numerical simulations of laminar and turbulent forced-convection heat transfer in channels with supercritical water. In 6th Int. Symposium on Supercritical Water-Cooled Reactors.

Bae, J. H., Yoo, J. Y., and McEligot, D. M. (2008). Direct numerical simulation of heated $\mathrm{CO}_{2}$ flows at supercritical pressure in a vertical annulus at $\mathrm{Re}=8900$. Phys. Fluids, 20, 055108.

Brinkerhoff, J. R. (2014). Numerical Investigations of Instability and Transition in Attached and Separated Shear Layers. Ph.D. thesis, Carleton University, Ottawa, Canada.

Brinkerhoff, J. R., John, A. C., and Yaras, M. I. (2011). Direct numerical simulation of the effect of wall proximity on the stability of a laminar planar free shear layer. In Proceedings of the 10th Int. Symposium on Experimental and Computational Aerothermodynamics of Internal Flows.

Brinkerhoff, J. R., and Yaras, M. I. (2011a). Direct numerical simulation of the growth of coherent flow structures in a triggered turbulent spot. In 7th Int. 
Symposium on Turbulence and Shear Flow Phenomena.

Brinkerhoff, J. R., and Yaras, M. I. (2011b). Interaction of viscous and inviscid instability modes in separation-bubble transition. Phys. Fluids, 23, 124102.

Brinkerhoff, J. R., and Yaras, M. I. (2012). Direct numerical simulation of a square jet ejected transversely into an accelerating, laminar main flow. Flow Turbul. Combust., 89, 519-546.

Brinkerhoff, J. R., and Yaras, M. I. (2014). Numerical investigation of the generation and growth of coherent flow structures in a triggered turbulent spot. J. Fluid Mech., 759, 257-294.

Brown, G. L., and Roshko, A. (1974). On density effects and large structure in turbulent mixing layers. J. Fluid Mech., 64, 775-816.

Butler, K. M., and Farrell, B. F. (1993). Optimal perturbations and streak spacing in wall-bounded turbulent shear flow. Phys. Fluids, 5, 774-777.

Choi, H., and Moin, P. (1994). Effects of the computational time step on numerical solutions of turbulent flow. J. Comput. Phys., 113, 1-4.

Chong, M. S., Perry, A. E., and Cantwell, B. J. (1990). A general classification of three-dimensional flow fields. Phys. Fluids, 2, 765-777.

Chong, T. P., and Zhong, S. (2003). Development of turbulent wedges in favourable pressure gradients. AIAA Paper no. 2003-4245.

Coles, D., and Barker, S. J. (1975). Some remarks on a synthetic turbulent boundary layer. Turbulent Mixing in Nonreactive and Reactive Flows (ed. S.N.B. Murthy), $285-293$.

Davey, R. F., and Roshko, A. (1972). The effect of a density difference on shear-layer instability. J. Fluid Mech., 53, 523-543.

Djenidi, L., and Antonia, R. A. (1993). LDA measurements in low Reynolds number turbulent boundary layer. Exp. Fluids, 14, 280-288.

Drazin, P. G., and Reid, W. H. (2004). Hydrodynamic Stability. Cambridge University 
Press, Cambridge.

Dubief, Y., and Delcayre, F. (2000). On coherent-vortex identification in turbulence. J. Turbulence, $1,11$.

Duffey, R. B., and Pioro, I. L. (2005). Experimental heat transfer of supercritical carbon dioxide flowing inside channels (survey). Nucl. Eng. Des., 235, 913-924.

Durbin, P., and Wu, X. (2007). Transition beneath vortical disturbances. Annu. Rev. Fluid Mech., 39, 107-128.

Elofsson, P. A., Kawakami, M., and Alfredsson, P. H. (1999). Experiments on the stability of streamwise streaks in plane Poiseuille flow. Phys. Fluids, 11, 915930.

Emmons, H. W. (1951). The laminar-turbulent transition in a boundary layer Part I. J. Aero. Sci., 18, 490-498.

Escudier, M. P., Abdel-Hameed, A., Johnson, M. W., and Sutcliffe, C. J. (1998). Laminarisation and re-transition of a turbulent boundary layer subjected to favourable pressure gradient. Exp. Fluids, 25, 491-502.

Gad-el-Hak, M., Blackwelder, R. F., and Riley, J. J. (1981). On the growth of turbulent regions in laminar boundary layers. J. Fluid Mech., 110, 73-95.

Ganapathisubramini, B., Longmire, E. K., and Marusic, I. (2003). Characteristics of vortex packets in turbulent boundary layers. J. Fluid Mech., 478, 35-46.

Glezer, A., Katz, Y., and Wygnanski, I. (1989). On the breakdown of the wave packet trailing a turbulent spot in a laminar boundary layer. J. Fluid Mech., 198, 1-26.

Gostelow, J. P., Melwani, N., and Walker, G. J. (1996). Effects of streamwise pressure gradient on turbulent spot development. J. Turbomach., 118, 737-743.

Hazel, P. (1972). Numerical studies of the stability of inviscid stratified shear flows. J. Fluid Mech., 51, 39-61. 
Head, M. R., and Bandyopadhyay, P. (1981). New aspects of turbulent boundarylayer structure. J. Fluid Mech., 107, 297-338.

Henningson, D., Spalart, P., and Kim, J. (1987). Numerical simulations of turbulent spots in plane Poiseuille and boundary-layer flow. Phys. Fluids, 30, 2914-2917.

Ho, C.-H., and Huerre, P. (1984). Perturbed free shear layers. Annu. Rev. Fluid Mech., 16, 365-424.

Honkan, A., and Andreopoulos, Y. (1997). Vorticity, strain-rate and dissipation characteristics in the near-wall region of turbulent boundary layers. J. Fluid Mech., 350, 29-96.

John, A. (2012). A Numerical Study of the Stability of Two-Dimensional Laminar Shear Layers. Master's thesis, Carleton University, Ottawa, Canada.

Jones, W. P., and Launder, B. E. (1972). Some properties of sink-flow turbulent boundary layers. J. Fluid Mech., 56, 337-351.

Katz, Y., Seifert, A., and Wygnanski, I. (1990). On the evolution of the turbulent spot in a laminar boundary layer with a favourable pressure gradient. J. Fluid Mech., 221, 1-22.

Kline, S. J., Reynolds, W. C., Schraub, F. A., and Runstadler, P. W. (1967). The structure of turbulent boundary layers. J. Fluid Mech., 30, 741-773.

Lawrence, G. A., Browand, F. K., and Redekopp, L. G. (1991). The stability of a sheared density interface. Phys. Fluids, 3, 2360-2370.

Lesieur, M., Métais, O., and Comte, P. (2005). Large Eddy Simulation of Turbulence. Cambridge University Press, Cambridge.

Matsui, T. (1980). Visualization of turbulent spots in the boundary layer along a flat plate in a water flow. In Proceedings of the IUTAM Symposium on LaminarTurbulent Transition.

Monkewitz, P. A., and Huerre, P. (1982). Influence of the velocity ratio on the spatial instability of mixing layers. Phys. Fluids, 25, 1137-1143. 
Moretti, P. M., and Kays, W. M. (1965). Heat transfer to a turbulent boundary layer with varying free-stream velocity and varying surface temperature - an experimental study. Int. J. Heat Mass Trans., 8, 1187-1202.

Özgen, S. (2004). Effect of heat transfer on stability and transition characteristics of boundary-layers. Int. J. Heat Mass Trans., 47, 4697-4712.

Perry, A. E., Lim, T. T., and Teh, E. W. (1981). A visual study of turbulent spots. J. Fluid Mech., 104, 387-405.

Pioro, I. L., Khartabil, H. F., and Duffey, R. B. (2004). Heat transfer to supercritical fluids flowing in channels - empirical correlations (survey). Nucl. Eng. Des., 230, 69-91.

Reddy, S. C., Schmid, P. J., Baggett, J. S., and Henningson, D. S. (1998). On stability of streamwise streaks and transition thresholds in plane channel flows. J. Fluid Mech., 365, 269-303.

Redjem-Saad, L., Ould-Rouiss, M., and Lauriat, G. (2007). Direct numerical simulation of turbulent heat transfer in pipe flows: effect of Prandtl number. Int. J. Heat Fluid Fl., 28, 847-861.

Reinaud, J., Joly, L., and Chassaing, P. (1999). Numerical simulation of a variabledensity mixing-layer. In Proceedings of the 3rd Int. Workshop on Vortex Flows and Related Numerical Methods (Vol. 7, p. 359-368).

Reinaud, J., Joly, L., and Chassaing, P. (2000). The baroclinic secondary instability of the two-dimensional shear layer. Phys. Fluids, 12, 2489-2505.

Roache, P. J. (1994). Perspective: a method for uniform reporting of grid refinement studies. J. Fluid Eng., 116, 405-413.

Sankaran, R., Chambers, A. J., and Antonia, R. A. (1986). The influence of a favourable pressure gradient on the growth of a turbulent spot. In 9th Australasian Fluid Mechanics Conference.

Schäfer, P., Severin, J., and Herwig, H. (1995). The effect of heat transfer on the 
stability of laminar boundary layers. Int. J. Heat Mass Trans., 38, 1855-1863.

Schröder, A., Geisler, R., Elsinga, G. E., Scarano, F., and Dierksheide, U. (2008). Investigation of a turbulent spot and a tripped turbulent boundary layer flow using time-resolved tomographic PIV. Exp. Fluids, 44, 305-316.

Schröder, A., and Kompenhans, J. (2004). Investigation of a turbulent spot using multi-plane stereo particle image velocimetry. Exp. Fluids, 36, 82-90.

Schubauer, G. B., and Klebanoff, P. S. (1956). Contributions on the mechanics of boundary-layer transition. NACA Report 1289.

Singer, B. A. (1996). Characteristics of a young turbulent spot. Phys. Fluids, 8, 509-521.

Singer, B. A., and Joslin, R. D. (1994). Metamorphosis of a hairpin vortex into a young turbulent spot. Phys. Fluids, 6, 3724-3736.

Smith, C. R., and Metzler, S. P. (1983). The characteristics of low-speed streaks in the near-wall region of a turbulent boundary layer. J. Fluid Mech., 129, 27-54.

Smith, C. R., Walker, J. D. A., Haidari, A. H., and Sobrun, U. (1991). On the dynamics of near-wall turbulence. Phil. Trans. R. Soc. Lond. A, 336, 131-175.

Smyth, W. D., and Peltier, W. R. (1989). The transition between Kelvin-Helmholtz and Holmboe instability: an investigation of the overreflection hypothesis. $J$. Atmos. Sci., 46, 3698-3720.

Soteriou, M. C., and Ghoniem, A. F. (1995). Effects of the free-stream density ratio on free and forced spatially developing shear layers. Phys. Fluids, 7, 2036-2051.

Spalart, P. R. (1988). Direct simulation of a turbulent boundary layer up to $\operatorname{Re}_{\theta}=1410$. J. Fluid Mech., 187, 61-98.

Stanislas, M., Perret, L., and Foucaut, J.-M. (2008). Vortical structures in the turbulent bounday layer: a possible route to a universal representation. $J$. Fluid Mech., 602, 327-382.

Staquet, C. (1995). Two-dimensional secondary instabilities in a strongly stratified 
shear layer. J. Fluid Mech., 296, 73-126.

Swearingen, J. D., and Blackwelder, R. F. (1987). The growth and breakdown of streamwise vortices in the presence of a wall. J. Fluid Mech., 182, 255-290.

Tomkins, C. D., and Adrian, R. J. (2003). Spanwise structure and scale growth in turbulent boundary layers. J. Fluid Mech., 490, 37-74.

Turner, J. S. (1973). Buoyancy Effects in Fluids. Cambridge University Press, Cambridge.

Wazzan, A. R., Keltner, G., Okamura, T. T., and Smith, A. M. O. (1972). Spatial stability of stagnation water boundary layer with heat transfer. Phys. Fluids, 15, 2114-2118.

Wu, X., Jacobs, R. G., Hunt, J. C. R., and Durbin, P. A. (1999). Simulation of boundary layer transition induced by periodically passing wakes. J. Fluid Mech., 398, 109-153.

Wygnanski, I. (1981). The effects of Reynolds number and pressure gradient on the transitional spot in a laminar boundary layer. Lecture Notes in Physics: The Role of Coherent Structures in Modelling Turbulence and Mixing, 136, 304-332.

Wygnanski, I., Sokolov, M., and Friedman, D. (1976). On a turbulent 'spot' in a laminar boundary layer. J. Fluid Mech., 78, 785-819.

Yaras, M. I. (2007). An experimental study of artificially-generated turbulent spots under strong favorable pressure gradients and freestream turbulence. J. Fluids Eng., 129, 563-572.

Zaki, T. A., and Durbin, P. A. (2005). Mode interaction and the bypass route to transition. J. Fluid Mech., 531, 85-111.

Zhong, S., Kittichaikarn, C., Hodson, H. P., and Ireland, P. T. (1998). Visualization of turbulent spots under the influence of adverse pressure gradients. In Proceedings of the 8th Int. Conference on Flow Visualization.

Zhou, J., Adrian, R. J., Balachandar, S., and Kendall, T. M. (1999). Mechanisms for 
generating coherent packets of hairpin vortices in channel flow. J. Fluid Mech., 387, 353-396. 


\section{Appendix A}

\section{Derivation of the Expression for the Rate of Baroclinic Vorticity Generation}

The expression for the rate of baroclinic vorticity generation is obtained from the variable-property vorticity transport equation. The vorticity transport equation is found by taking the curl of the non-conservation form of the Navier-Stokes equation with external body forces neglected:

$$
\begin{gathered}
\nabla \times\left(\rho \frac{\partial \mathbf{U}}{\partial t}+\rho(\mathbf{U} \cdot \nabla) \mathbf{U}=-\nabla P+\nabla \cdot \tau\right) \\
\nabla \rho \times \frac{\partial \mathbf{U}}{\partial t}+\rho \frac{\partial(\nabla \times \mathbf{U})}{\partial t}+\nabla \rho \times(\mathbf{U} \cdot \nabla) \mathbf{U}+\rho \nabla \times(\mathbf{U} \cdot \nabla) \mathbf{U}=-\nabla \times \nabla P+\nabla \times \nabla \cdot \tau
\end{gathered}
$$

Let $\Omega=\nabla \times \mathbf{U}$. Also, the following vector calculus identities are noted, where $\mathbf{A}$ and $\mathbf{B}$ are vectors and $A$ is a scalar:

$$
\begin{gathered}
(\mathbf{A} \cdot \nabla) \mathbf{A}=\nabla\left(\frac{1}{2} \mathbf{A} \cdot \mathbf{A}\right)-\mathbf{A} \times(\nabla \times \mathbf{A}) \\
\nabla \times(\mathbf{A} \times \mathbf{B})=\mathbf{A}(\nabla \cdot \mathbf{B})-\mathbf{B}(\nabla \cdot \mathbf{A})+(\mathbf{B} \cdot \nabla) \mathbf{A}-(\mathbf{A} \cdot \nabla) \mathbf{B} \\
\nabla \cdot(\nabla \times \mathbf{A})=0
\end{gathered}
$$


$\mathbf{A} \times \mathbf{B}=-\mathbf{B} \times \mathbf{A}$

$$
\nabla \times \nabla A=0
$$

Substitution of these relations into Eqn. A.2 yields:

$$
\begin{aligned}
\nabla \rho \times \frac{\partial \mathbf{U}}{\partial t}+\rho \frac{\partial \boldsymbol{\Omega}}{\partial t} & +\nabla \rho \times(\mathbf{U} \cdot \nabla) \mathbf{U}+ \\
& \nabla \times(\underbrace{\nabla\left(\frac{1}{2} \mathbf{U} \cdot \mathbf{U}\right)-\mathbf{U} \times \boldsymbol{\Omega}}_{\text {Eqn. A.3 }})=-\underbrace{\nabla \times \nabla P}_{\begin{array}{c}
=0 \text { by } \\
\text { Eqn. A.7 }
\end{array}}+\nabla \times \nabla \cdot \tau
\end{aligned}
$$

$$
\begin{aligned}
\rho \frac{\partial \boldsymbol{\Omega}}{\partial t}+\nabla \rho \times \overbrace{\left(\frac{\partial \mathbf{U}}{\partial t}+(\mathbf{U} \cdot \nabla) \mathbf{U}\right)}^{=D \mathbf{U} / D t}+ \\
\quad(\underbrace{\nabla \times \nabla\left(\frac{1}{2} \mathbf{U} \cdot \mathbf{U}\right)}_{=0 \text { by Eqn. A.7 }}-\nabla \times(\mathbf{U} \times \mathbf{\Omega}))=\nabla \times \nabla \cdot \tau
\end{aligned}
$$

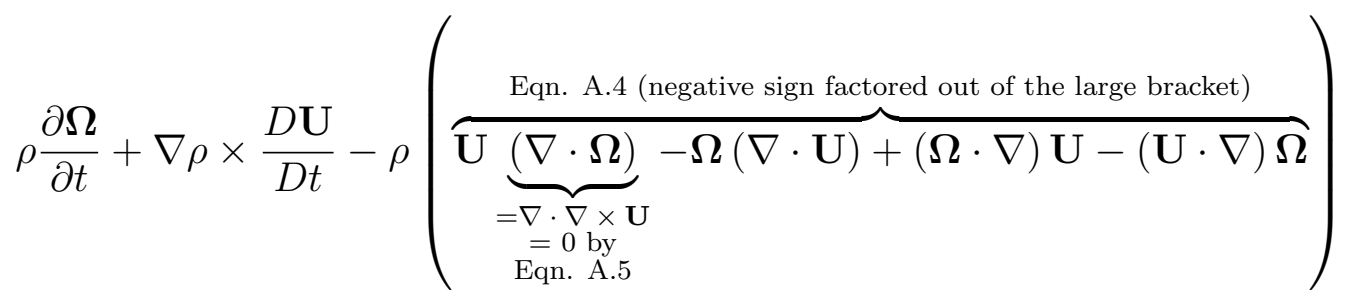

$$
\begin{aligned}
& =\nabla \times \nabla \cdot \tau
\end{aligned}
$$


Rearranging Eqn. A.10 and dividing by $\rho$, the final form of the variable-property compressible vorticity transport equation is obtained:

$$
\underbrace{\frac{\partial \boldsymbol{\Omega}}{\partial t}+(\mathbf{U} \cdot \nabla) \boldsymbol{\Omega}}_{\mathrm{A}}=\underbrace{(\boldsymbol{\Omega} \cdot \nabla) \mathbf{U}}_{\mathrm{B}}-\underbrace{\boldsymbol{\Omega}(\nabla \cdot \mathbf{U})}_{\mathrm{C}}+\underbrace{\frac{1}{\rho}\left(\frac{D \mathbf{U}}{D t} \times \nabla \rho\right)}_{\mathrm{D}}+\underbrace{\frac{1}{\rho}(\nabla \times \nabla \cdot \tau)}_{\mathrm{E}}
$$

Term $\mathrm{A}$ is the material derivative of the vorticity vector and term $\mathrm{E}$ accounts for viscous diffusion of vorticity. Terms B, C, and D represent three vorticity production terms. Term B represents the rate of vorticity production by stretching and tilting of vorticity due to gradients in velocity. The production rate due to stretching of vorticity due to compressibility is denoted by term C. Finally, term D is the production rate of vorticity by the baroclinic mechanism. 


\section{Appendix B}

\section{Data Analysis Procedures}

This appendix briefly describes the data reduction procedures used in the present study. This includes calculation of perturbation quantities, differential and integral flow parameters, and the second invariant of the velocity gradient tensor.

\section{B.1 Perturbation quantities}

The perturbation velocity field is calculated by subtracting the undisturbed velocity field, which is obtained from a wall-normal-streamwise plane in the laminar boundary layer, from the instantaneous velocity field:

$$
\begin{gathered}
u(x, y, z, t)=U(x, y, z, t)-U(x, y)_{\text {undisturbed }} \\
v(x, y, z, t)=V(x, y, z, t)-V(x, y)_{\text {undisturbed }} \\
w(x, y, z, t)=W(x, y, z, t)-W(x, y)_{\text {undisturbed }}
\end{gathered}
$$


Components of perturbation vorticity are then calculated directly from components of perturbation velocity:

$$
\begin{aligned}
& \omega_{x}=\frac{\partial w}{\partial y}-\frac{\partial v}{\partial z} \\
& \omega_{y}=\frac{\partial u}{\partial z}-\frac{\partial w}{\partial x} \\
& \omega_{z}=\frac{\partial v}{\partial x}-\frac{\partial u}{\partial y}
\end{aligned}
$$

\section{B.2 Differential flow parameters}

Differential quantities such as those in Eqn. B.2 are computed using a centreddifferencing scheme. In the $\mathrm{x}$ and $\mathrm{z}$ directions, derivatives are evaluated as:

$$
\left.\frac{\partial \phi}{\partial x}\right|_{i}=\frac{\phi_{i+1}-\phi_{i-1}}{x_{i+1}-x_{i-1}}
$$

where $\phi$ is a generic flow variable, $i$ is the node at which the derivative is evaluated, and the derivative is evaluated in the $\mathrm{x}$ direction as an example. At nodes along boundaries, first-order forward- or backward-differencing schemes are used as applicable.

Because of large wall-normal gradients in flow parameters and non-uniform node spacing, in the y direction a quadratic curve fit is applied to $y_{i-1}, y_{i}$, and $y_{i+1}$. The derivative is taken of the curve fit and evaluated at $y_{i}$ to yield the following centreddifferencing expression for the derivative at node $i$ :

$$
\begin{aligned}
\left.\frac{\partial \phi}{\partial y}\right|_{i}=\phi_{i-1} \frac{y_{i}-y_{i+1}}{\left(y_{i-1}-y_{i}\right)\left(y_{i-1}-y_{i+1}\right)}+ & \\
& \phi_{i} \frac{2 y_{i}-y_{i-1}-y_{i+1}}{\left(y_{i}-y_{i+1}\right)\left(y_{i}-y_{i-1}\right)}+\phi_{i+1} \frac{y_{i}-y_{i-1}}{\left(y_{i-1}-y_{i+1}\right)\left(y_{i}-y_{i+1}\right)}
\end{aligned}
$$

At boundary nodes, derivatives in the y direction are evaluated using the following 
forward- and backward-differencing expressions, respectively, similar to the centreddifferencing expression in Eqn. B.4:

$$
\begin{aligned}
& \left.\frac{\partial \phi}{\partial y}\right|_{i}=\phi_{i} \frac{2 y_{i}-y_{i+1}-y_{i+2}}{\left(y_{i}-y_{i+1}\right)\left(y_{i}-y_{i+2}\right)}+ \\
& \phi_{i+1} \frac{y_{i}-y_{i+2}}{\left(y_{i+1}-y_{i}\right)\left(y_{i+1}-y_{i+2}\right)}+\phi_{i+2} \frac{y_{i}-y_{i+1}}{\left(y_{i}-y_{i+2}\right)\left(y_{i+1}-y_{i+2}\right)} \\
& \left.\frac{\partial \phi}{\partial y}\right|_{i}=\phi_{i-2} \frac{y_{i}-y_{i-1}}{\left(y_{i-2}-y_{i-1}\right)\left(y_{i-2}-y_{i}\right)}+ \\
& \phi_{i-1} \frac{y_{i}-y_{i-2}}{\left(y_{i-1}-y_{i}\right)\left(y_{i-1}-y_{i-2}\right)}+\phi_{i} \frac{2 y_{i}-y_{i-2}-y_{i-1}}{\left(y_{i-2}-y_{i}\right)\left(y_{i-1}-y_{i}\right)}
\end{aligned}
$$

\section{B.3 Integral flow parameters}

The velocity boundary layer thickness, $\delta$, is identified as the location at which the local streamwise velocity magnitude exceeds $99 \%$ of the local freestream velocity, and the thermal boundary layer thickness is evaluated as the location at which the local temperature is within $0.1 \mathrm{~K}$ of the local freestream temperature. The local boundary layer displacement thickness, $\delta^{*}$, and momentum thickness, $\theta$, are respectively computed using the following two expressions:

$$
\begin{gathered}
\delta^{*}=\int_{0}^{\delta}\left(1-\frac{\rho(y) U(y)}{\rho_{\infty} U_{\infty}}\right) d y \\
\theta=\int_{0}^{\delta} \frac{\rho(y) U(y)}{\rho_{\infty} U_{\infty}}\left(1-\frac{U(y)}{U_{\infty}}\right) d y
\end{gathered}
$$

The free shear layer momentum thickness is evaluated as:

$$
\theta_{s}=\int_{-\delta}^{+\delta} \frac{\rho(y) U(y)-(\rho U)_{\min }}{\rho_{1} U_{1}-(\rho U)_{\min }}\left(1-\frac{U(y)-U_{\min }}{U_{1}-U_{\min }}\right) d y
$$


where $\pm \delta$ are the farthest locations relative to the splitter-plate $\mathrm{y}$-coordinate where the difference between the local streamwise velocity and the freestream velocity in the respective stream exceeds $1 \%$. Parameters with subscripts ' 1 ' and 'min' correspond to freestream values in the high-speed stream and minimum values in the free shear layer, respectively.

Significant wall-normal variation in the kinematic viscosity is present in the boundary layer in the turbulent spot simulations of the present study. Therefore, a spatiallyaveraged value of kinematic viscosity is used in the evaluation of the acceleration parameter and Reynolds number:

$$
\bar{\nu}=\frac{1}{\delta} \int_{0}^{\delta} \nu(y) d y
$$

Since all integral quantities in the present study are evaluated in the y direction where the node spacing is finest, evaluation of integral quantities using the following approximation was found to be sufficiently accurate:

$$
\int_{a}^{b} \phi(y) d y=\sum_{i=2}^{N} \phi_{i}\left(y_{i}-y_{i-1}\right)
$$

\section{B.4 Identification of coherent vortical structures of turbu- lence}

Coherent vortical structures of turbulence are visualized in the present study using the second invariant of the velocity gradient tensor, denoted $Q$. Lesieur et al. (2005) define coherent vortical structures of turbulence as regions with a high concentration of vorticity with a low static pressure core, such that the fluid particles rotating about the vortex are relatively balanced between centrifugal and pressure gradient forces. Dubief and Delcayre (2000) and Lesieur et al. (2005) mathematically demonstrate 
that a necessary condition for the presence of a vortex tube with a low-pressure core is $Q>0$.

Brinkerhoff (2014) determined that turbulence structures are most clearly visualized when $Q$ is calculated using the perturbation velocity flow field. The perturbation velocity flow field can be expressed as:

$$
\mathbf{u}=\mathbf{A x}
$$

or

$$
\left(\begin{array}{c}
u \\
v \\
w
\end{array}\right)=\left(\begin{array}{ccc}
\frac{\partial u}{\partial x} & \frac{\partial u}{\partial y} & \frac{\partial u}{\partial z} \\
\frac{\partial v}{\partial x} & \frac{\partial v}{\partial y} & \frac{\partial v}{\partial z} \\
\frac{\partial w}{\partial x} & \frac{\partial w}{\partial y} & \frac{\partial w}{\partial z}
\end{array}\right)\left(\begin{array}{l}
x \\
y \\
z
\end{array}\right)
$$

where $\mathbf{A}$ is the velocity gradient tensor. The second invariant of $\mathbf{A}$ is therefore (Chong et al., 1990):

$$
\begin{gathered}
Q=\left|\begin{array}{cc}
\frac{\partial u}{\partial x} & \frac{\partial u}{\partial y} \\
\frac{\partial v}{\partial x} & \frac{\partial v}{\partial y}
\end{array}\right|+\left|\begin{array}{cc}
\frac{\partial u}{\partial x} & \frac{\partial u}{\partial z} \\
\frac{\partial w}{\partial x} & \frac{\partial w}{\partial z}
\end{array}\right|+\left|\begin{array}{cc}
\frac{\partial v}{\partial y} & \frac{\partial v}{\partial z} \\
\frac{\partial w}{\partial y} & \frac{\partial w}{\partial z}
\end{array}\right| \\
Q=\frac{\partial u}{\partial x} \frac{\partial v}{\partial y}-\frac{\partial u}{\partial y} \frac{\partial v}{\partial x}+\frac{\partial u}{\partial x} \frac{\partial w}{\partial z}-\frac{\partial u}{\partial z} \frac{\partial w}{\partial x}+\frac{\partial v}{\partial y} \frac{\partial w}{\partial z}-\frac{\partial v}{\partial z} \frac{\partial w}{\partial y} \\
Q=\frac{1}{2}\left(\frac{\partial u_{i}}{\partial x_{i}} \frac{\partial u_{j}}{\partial x_{j}}-\frac{\partial u_{i}}{\partial x_{j}} \frac{\partial u_{j}}{\partial x_{i}}\right)
\end{gathered}
$$

